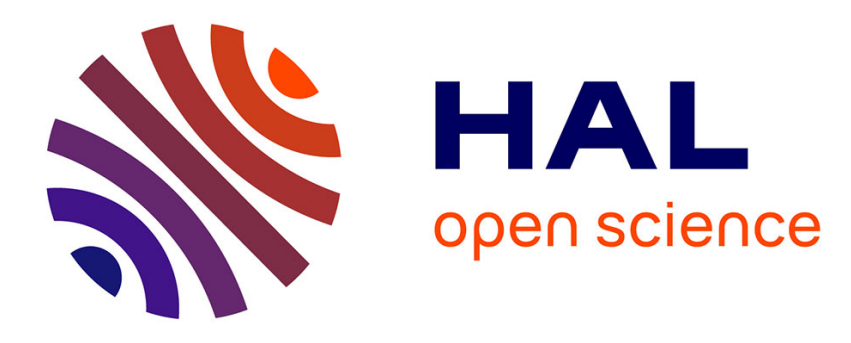

\title{
Feedback control of multibody systems with joint clearance and dynamic backlash: a tutorial \\ Bernard Brogliato
}

\section{To cite this version:}

Bernard Brogliato. Feedback control of multibody systems with joint clearance and dynamic backlash: a tutorial. Multibody System Dynamics, 2018, 42 (3), pp.283-315. 10.1007/s11044-017-9585-4 . hal01499581v2

\section{HAL Id: hal-01499581 \\ https://hal.inria.fr/hal-01499581v2}

Submitted on 6 Nov 2017

HAL is a multi-disciplinary open access archive for the deposit and dissemination of scientific research documents, whether they are published or not. The documents may come from teaching and research institutions in France or abroad, or from public or private research centers.
L'archive ouverte pluridisciplinaire $\mathbf{H A L}$, est destinée au dépôt et à la diffusion de documents scientifiques de niveau recherche, publiés ou non, émanant des établissements d'enseignement et de recherche français ou étrangers, des laboratoires publics ou privés. 


\title{
Feedback control of multibody systems with joint clearance and dynamic backlash: A tutorial
}

\author{
Bernard Brogliato
}

Received: date / Accepted: date

\begin{abstract}
The problem of feedback control of mechanisms with joint clearance is analysed. Various control strategies are reviewed: impactless trajectories with persistent contact, control through collisions, the stabilization of equilibrium points, and trajectory tracking control. This article sets a general control framework, brings some preliminary answers and leaves some problems open, which are mentionned all through the article and in the conclusions.
\end{abstract}

\section{Introduction}

Lagrangian mechanical systems subject to unilateral constraints, impacts and friction, make a rich class of nonsmooth, nonlinear dynamical systems. Though a lot remains to be discovered, it seems that constrained manipulators, biped robots, kinematic chains with joint clearance, juggling systems, tensegrity (cable-driven) and tethered systems, etc, possess different stability and control properties. It is therefore necessary to analyse the control of each subclass separately. Within the framework of multi-rigid-body systems with joint clearance, the main modelling issue concerns the choice of the contact model. Clearances involve additional degrees of freedom and introduce unilateral constraints. There are several available classes of models for collisions between bodies in the Mechanical Engineering literature, see e.g. [18, Chapters 2, 4, 5, 6] and [32, Chapter 3]. Commonly used two-body impact models in the Multibody System's literature, are extensions of Hertz' contact with added dissipation, the most well-known being the Simon-Hunt-Crossley and the Kuwabara-Kono's dissipations, as well as their many variants like bistiffness Lankarani-Nikravesh and Walton-Braun models [18, §2.2.2, §4.2.1.2]. The main issue is that clearances may involve conformal contacting surfaces, which do not satisfy basic requirements for Hertz' elasticity (see [88, Table 2] for plane/plane contact elasticity coefficient). Moreover the dissipation modelling is also a tough issue, and it is not clear whether or not nonlinear viscosity may be a suitable model. Advantages of compliant models, is that they allow for the contact force history computation, however this is not necessarily of interest for the design of feedback controllers, especially if the impact duration $t_{f}$ is very short and prevents the actuators from being active during collisions (linear spring-dashpot yields $t_{f}=\mathcal{O}\left(\frac{1}{\sqrt{k}}\right)$, Hertz contact yields $t_{f}=\mathcal{O}\left(k_{h}^{-\frac{2}{5}}\left|v_{\mathrm{n}}\left(t_{0}^{-}\right)\right|^{-\frac{1}{5}}\right)$ [18], steel/steel collisions may have durations of a millisecond or less). Drawbacks are that they may involve too many parameters which are difficult to estimate in practice, and that they may induce stiff differential equations during persistent contact phases of motion, rendering the numerical simulation long and delicate. In particular the constraint stabilization issue is not easy, and spurious oscillations due to the model and the numerical method may appear during persistent contact modes (compare for instance the experimental and numerical results for the slider acceleration of a slider/crank system with a clearance in a revolute joint [33, Figures 11,12]

INRIA Grenoble Rhône-Alpes and Laboratoire Jean Kuntzman, University Grenoble-Alpes, 655 avenue de l'Europe, 38334 Saint-Ismier, France. E-mail: bernard.brogliato@inria.fr 
and [42, Figures 5,6,7,8]). See for instance [32, Chapter 2] for more informations on numerical issues. The modelling of three-dimensional revolute joints is even a harder task, see [89] and [70] for comparisons between various cylindrical bearing/journal impact models, showing the great difficulty caused by such contacts.

Another class of contact/impact models relies on the use of (possibly non constant) coefficients of restitution to describe instantaneous impacts, and model persistent contact with holonomic bilateral (equality) constraints. These models use properly defined signed distance or gap functions $[18,32,35,72]$, and yield so-called complementarity conditions between the contact force and the distance between the two bodies that constitute the joint clearance. For planar prismatic joints, they may involve so-called multiple impacts similar to the rocking block system with line/line or plane/plane collisions, hence requiring specific impact laws [18, Chapter 6] [61,72]. Moreover friction may play a significant role in rigid-body prismatic joints, where jamming effects and Painlevé paradoxes singularities may occur $[18, \S 5.6]$. Let $q \in \mathbb{R}^{n}$ denote the vector of independent generalized coordinates for the system with open clearances and no bilateral constraints, $f_{i}(q)$ denote the $i$ th gap function, and $\lambda_{\mathrm{n}, u, i}$ denote the Lagrange multiplier associated with the unilateral constraint $f_{i}(q) \geqslant 0$. Then the complementarity conditions are $0 \leqslant f_{i}(q) \perp \lambda_{\mathrm{n}, u, i} \geqslant 0$ and stem from very basic and natural modelling assumptions [18, §5.4.1]. When inserted in the dynamics together with an impact law, complementarity conditions yield nonsmooth mechanical systems that are numerically tractable with time-stepping methods [2], and which have been proved to possess very good predictability for systems with clearance joints $[43,82]$ as well as good parameter (restitution and friction coefficients) identifiability [81]. In particular, constraint stabilization to avoid constraint drift can be very efficiently dealt with [1], so that persistent contact modes are simulated without oscillations and drift. To summarize, multibody systems with joint clearance represented as Lagrangian systems with $m_{b}$ frictionless bilateral holonomic constraints $h(q)=0$ and associated Lagrange multiplier $\lambda_{\mathrm{n}, b}, m_{u}$ frictionless unilateral constraints and complementarity conditions, an impact law, and a control torque input $\tau \in \mathbb{R}^{p}$, have the dynamical equations:

$$
\left\{\begin{array}{l}
M(q) \ddot{q}+C(q, \dot{q}) \dot{q}+G(q)=E(q) \tau+\nabla f(q) \lambda_{\mathrm{n}, u}+\nabla h(q) \lambda_{\mathrm{n}, b} \\
0 \leqslant f(q) \perp \lambda_{\mathrm{n}, u} \geqslant 0, \quad h(q)=0 \\
\text { Impact law (kinetic or kinematic coefficients of restitution) }
\end{array}\right.
$$

for some matrix $E(q) \in \mathbb{R}^{n \times p}, p \geqslant 1, M(q)=M(q)^{T} \succ 0$ is the inertia matrix, $C(q, \dot{q}) \dot{q}$ contains centripetal and Coriolis forces, $G(q)$ represents forces that derive from a smooth potential (gravity, elasticity, etc $)^{1}$. The bilateral constraints account for joints without clearance if the coordinates are not reduced. The gradients $\nabla f(q) \in \mathbb{R}^{n \times m_{u}}$ and $\nabla h(q) \in \mathbb{R}^{n \times m_{b}}$. The admissible domain is a subset of the configuration space $\mathcal{C} \supseteq \Phi=\{q \in \mathcal{C} \mid h(q)=0, f(q) \geqslant 0\}$. The dynamical system in (1) is a complex nonlinear nonsmooth system, see e.g. [18, Chapters 2 and 5] for well-posedness results. Relying on [18, Theorem 5.3] we will assume throughout the paper that systems as in (1) have absolutely continuous positions $q(\cdot)$, velocities $\dot{q}(\cdot)$ are right-continuous of local bounded variations ${ }^{2}$, and acceleration are measures, for all piecewise analytic inputs $\tau(\cdot)$. The relationships between (1) and Lagrangian systems with compliant contact/impact models, is a tough mathematical issue which has nevertheless been analysed in some cases [18, Chapter 2] [69].

All the above models have been proposed and studied in the Mechanical Engineering literature. In the Control literature systems with clearances are called systems with backlash. The most studied models consider clearances as in Figure 2 (a) (b). Most often they represent mechanical play as static backlash with dead-zone or hysteresis [63], which have the advantage of being tractable for control design, but totally neglect all dynamical phenomena like collisions. Consequently they are likely to be realistic in a very narrow range of small velocities [30]. In a multibody systems perspective where many applications may involve high velocities, dynamical effects are expected to be significant. The feedback stabilization of systems with clearances in one-dimensional prismatic joints (impact oscillators), using (1) as a dynamical

\footnotetext{
1 In general, the dynamics could be written using Newton-Euler formalism. However for control purpose the Lagrangian framework is the most suitable one, see e.g. [19].

2 Hence, $\dot{q}\left(t^{+}\right)=\dot{q}(t)$ for all $t \geqslant 0$.
} 
framework, has been tackled in [57] inspired by juggling robots control [22], circumventing the underactuated feature through the use of impacts. Trajectory tracking for the impact oscillator has been studied in [53], using similar dead-beat control ideas. Compliant elastic contact in one DoF (Degree of Freedom) clearances are considered in $[44,62]$ for estimation and control applications. Specific "pre-loading" controllers that guarantee journal/bearing persistent contact in parallel manipulators with redundant control are studied in [60] (redundancy is understood here from the point of view of the clearance-free system). Clearances are modeled with bilateral holonomic constraints in [60], while we use a more realistic approach in this paper. We do not review structural optimization (like mass re-distribution to reduce impacts [83]) issues in this introduction, which may be seen as preliminary and complementary to control design. See also the introduction in [4] for an overview of control of systems with backlash.

In this article we mainly use the complementarity framework in (1), which is shown to provide a suitable framework for Control analysis. Section 2 settles the dynamical framework. Section 3 is dedicated to the analysis of impactless, persistent contact control. Section 4 deals with control with impacts, where mechanisms with clearances are recast into juggling systems. Section 5 examines the stabilization of equilibrium points issue, while section 6 is devoted to the trajectory tracking problem, using PD+ controllers. Conclusions are in section 7.

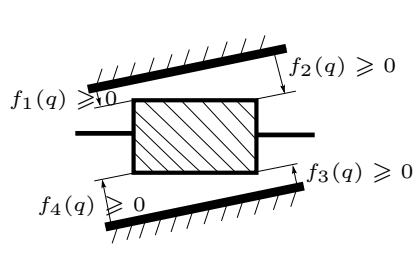

(a)

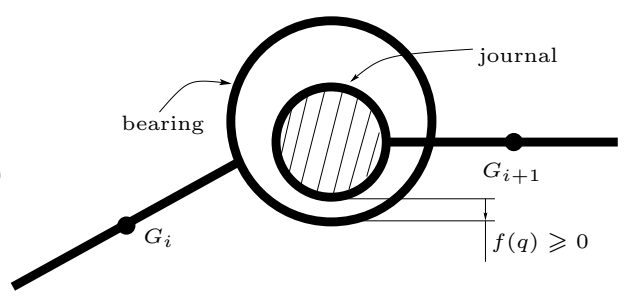

(b)

Fig. 1: Clearances in (a) planar prismatic and (b) pivot joints.

(a)

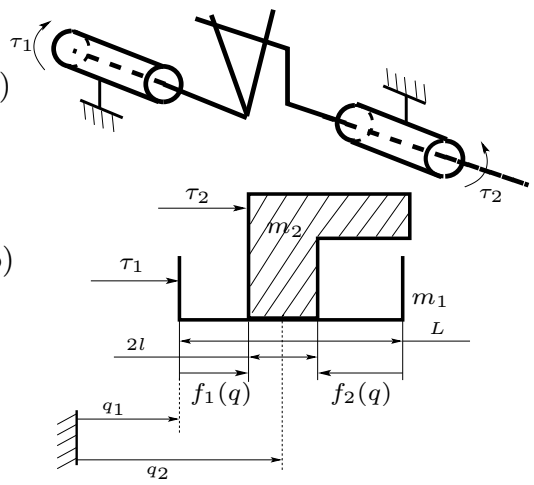

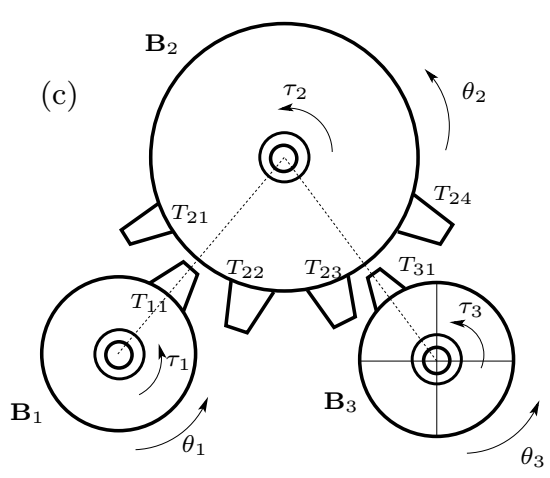

Fig. 2: Clearance models in: (a) pivot joints with gear backlash, (b) one-DoF prismatic, (c) gears.

Mathematical notation: Following [59], let $K=\left\{x \in \mathbb{R}^{n} \mid f_{i}(x) \geqslant 0,1 \leqslant i \leqslant m\right\} \subseteq \mathbb{R}^{n}$ be a finitely represented set. The tangent cone (linearization cone) to $K$ at $x$ is defined as $T_{K}(x)=\left\{v \in \mathbb{R}^{n} \mid v^{T} \nabla f_{i}(q) \geqslant\right.$ 
$0, i \in \mathcal{I}(x)\}$, where $\mathcal{I}(x)=\left\{i \in\{1, m\} \mid f_{i}(x)=0\right\}$ is the index set of active constraints (we do not denote the tangent cone $V(x)$ as in [59] to avoid confusion with Lyapunov functions). The normal cone (linearization cone) is the polar cone of the tangent cone: $N_{K}(x)=\left\{u \in \mathbb{R}^{n} \mid u^{T} v \leqslant 0\right.$ for all $\left.v \in T_{K}(x)\right\}$, it is the cone generated by the normals $\nabla f_{i}(x), i \in \mathcal{I}(x)$. Let $A \in \mathbb{R}^{n \times n}$ be a matrix, $\lambda_{\min }(A)$ is its smallest eigenvalue, $\lambda_{\max }(A)$ is its largest eigenvalue, $A$ is positive definite $(A \succ 0)$ if $x^{T} A x>0$ for all $x \neq 0$. The kernel of $A$ is denoted $\operatorname{ker}(A)$, its image as $\operatorname{im}(A)$. The $i$ th row of $A$ is denoted $A_{i}$. Let $f: \mathbb{R}^{n} \rightarrow \mathbb{R}^{m}$ be differentiable, then $\nabla f(x)=\frac{\partial f}{\partial x}^{T}(x) \in \mathbb{R}^{n \times m}$. Let $a_{1}, a_{2}, \ldots, a_{n}$ be reals, then $[a]=\operatorname{diag}\left(a_{i}\right)$. The identity matrix is $I_{n} \in \mathbb{R}^{n \times n}$. Given a function $f(\cdot)$ which has right and left limits everywhere, we denote $f\left(t^{+}\right)=\lim _{s \rightarrow t, s>t} f(s)$ its right-limit, $f\left(t^{-}\right)=\lim _{s \rightarrow t, s<t} f(s)$ its left-limit. The Dirac measure at $t$ is denoted $\delta_{t}$.

\section{Lagrangian complementarity systems framework}

\subsection{Generalities and examples}

Let us investigate more the structure of (1) for systems with joint clearances. Perfect (i.e. frictionless) planar prismatic and revolute joints suppress two DoFs each. Planar prismatic (respectively revolute) joints with clearance create four (respectively one) gap functions, see Figure 1, one-DoF prismatic joints and gears with clearance create two gap functions, see Figure 2. Consequently $m_{u}=4 n_{p r}+n_{\text {rev }}$ if there are $n_{p r}$ and $n_{r e v}$ prismatic and revolute joints with clearance, respectively. When there are no clearances, the system in (1) has $n-m_{b}-2 n_{r e v}-2 n_{p r} \geqslant 1$ DoFs. When clearances are modelled, and all contacts in the joint clearances are open $\left(f_{i}(q)>0\right.$ for all $\left.1 \leqslant i \leqslant m_{u}\right)$, the "open" system $\mathcal{S}$ has $n-m_{b}$ DoFs and is constituted of $n_{\text {ind }}$ independent subsystems, possibly at the price of opening one clearance-free joint and adding bilateral constraints: the mass matrix $M(q)=\operatorname{blockdiag}\left(M_{1}\left(q_{1}\right), M_{2}\left(q_{2}\right), \ldots, M_{n_{\text {ind }}}\left(q_{n_{\text {ind }}}\right)\right)$ is block-diagonal, and so is the matrix $C(q, \dot{q})=\operatorname{blockdiag}\left(C_{1}\left(q_{1}, \dot{q}_{1}\right), C_{2}\left(q_{2}, \dot{q}_{2}\right), \ldots, C_{n_{\text {ind }}}\left(q_{n_{\text {ind }}}, \dot{q}_{n_{\text {ind }}}\right)\right)$, while $g(q)=\left(g_{1}\left(q_{1}\right)^{T}, \ldots, g_{n_{\text {ind }}}\left(q_{n_{\text {ind }}}\right)^{T}\right)^{T}$. It follows that the term $\nabla f(q) \lambda_{\mathrm{n}, u}$ which represents the contact forces inside joint clearances, has a special structure as well. We may denote $\mathcal{S}=\left(S_{1}, \ldots, S_{n_{\text {ind }}}\right)$.

Example 1 Consider the kinematic chain in Figure 3, which is an academic example. It has fourteen bodies, eleven revolute joints and one prismatic joint without clearance, one prismatic joint $J_{16}$ and three revolute joints $J_{1}, J_{9}, J_{14}$, with clearance. The system without clearances has seven DoFs. When clearances are present the open system has fifteen DoFs, and $n_{\text {ind }}=3: \mathcal{S}=\left(S_{1}, S_{2}, S_{3}\right)$. The subsystem $S_{1}$ has seven DoFs, subsystems $S_{2}$ and $S_{3}$ have four DoFs each. Body 0 is the ground. We can therefore describe $S_{i}$ with a vector of generalized coordinates $q_{i} \in \mathbb{R}^{n_{i}}, n_{1}=7, n_{2}=n_{3}=4$. Following for instance the developments in $[18, \S 4.1]$, there exist matrices $\mathcal{M}_{i j}(\cdot)$ such that the generalized contact forces $\nabla f(q) \lambda_{\mathrm{n}, u}$ have the form:

$$
\begin{aligned}
& M_{1}\left(q_{1}\right) \ddot{q}_{1}+F_{1}\left(q_{1}, \dot{q}_{1}\right)=\mathcal{M}_{10}\left(q_{1}\right) \lambda_{\mathrm{n}, u, 10}+\mathcal{M}_{12}\left(q_{1}, q_{2}\right) \lambda_{\mathrm{n}, u, 12}+E_{1}\left(q_{1}\right) \tau \\
& M_{2}\left(q_{2}\right) \ddot{q}_{2}+F_{2}\left(q_{2}, \dot{q}_{2}\right)=\mathcal{M}_{21}\left(q_{1}, q_{2}\right) \lambda_{\mathrm{n}, u, 12}+\mathcal{M}_{23}\left(q_{1}, q_{2}\right) \lambda_{\mathrm{n}, u, 23}+E_{2}\left(q_{2}\right) \tau \\
& M_{3}\left(q_{3}\right) \ddot{q}_{3}+F_{3}\left(q_{3}, \dot{q}_{3}\right)=\mathcal{M}_{32}\left(q_{1}, q_{2}\right) \lambda_{\mathrm{n}, u, 23}+\mathcal{M}_{30}\left(q_{3}\right) \lambda_{\mathrm{n}, u, 30}+E_{3}\left(q_{3}\right) \tau
\end{aligned}
$$

where $F_{i}\left(q_{i}, \dot{q}_{i}\right) \triangleq C\left(q_{i}, \dot{q}_{i}\right) \dot{q}_{i}+g_{i}\left(q_{i}\right), E(q)=\left(E_{1}(q)^{T}, E_{2}(q)^{T}, E_{3}(q)^{T}\right)^{T}$, with the complementarity conditions:

$$
\begin{aligned}
& 0 \leqslant f_{10}\left(q_{1}\right) \perp \lambda_{\mathrm{n}, u, 10} \geqslant 0,0 \leqslant f_{12}\left(q_{1}, q_{2}\right) \perp \lambda_{\mathrm{n}, u, 12} \geqslant 0 \\
& 0 \leqslant f_{23}\left(q_{2}, q_{3}\right) \perp \lambda_{\mathrm{n}, u, 23} \geqslant 0, \quad 0 \leqslant f_{30}\left(q_{3}\right) \perp \lambda_{\mathrm{n}, u, 30} \geqslant 0
\end{aligned}
$$

where $\lambda_{\mathrm{n}, u, 10} \in \mathbb{R}, \lambda_{\mathrm{n}, u, 12} \in \mathbb{R}, \lambda_{\mathrm{n}, u, 23} \in \mathbb{R}, \lambda_{\mathrm{n}, u, 30} \in \mathbb{R}^{4}$. Let us denote $v_{\mathrm{n}, i j}(t)=\nabla f_{i j}\left(q_{i}, q_{j}\right)^{T} \dot{q}(t)$, and $\lambda_{\mathrm{n}, u, i j}=p_{\mathrm{n}, u, i j}(t) \delta_{t}$ at impact times. The impact law may be chosen as a Newton's law with complementarity:

$$
\begin{aligned}
& 0 \leqslant v_{\mathrm{n}, i j}\left(t^{+}\right)+e_{\mathrm{n}, i j} v_{\mathrm{n}, i j}\left(t^{-}\right) \perp p_{\mathrm{n}, u, i j}(t) \geqslant 0 \\
& \text { when } f_{i j}(q(t))=0 \text { and } v_{\mathrm{n}, i j}\left(t^{-}\right) \leqslant 0,
\end{aligned}
$$

for $i j=10,12,23,30$. It is noteworthy that the action/reaction law (Newton's third law) is taken into account in the right-hand side of (2), and that the gap functions Jacobian matrices satisfy: $\frac{\partial f_{10}}{\partial q}=$ 
$\left(\mathcal{M}_{10}\left(q_{1}\right)^{T}, 0, . ., 0\right), \frac{\partial f_{12}}{\partial q}=\left(\mathcal{M}_{12}\left(q_{1}, q_{2}\right)^{T}, \mathcal{M}_{21}\left(q_{1}, q_{2}\right)^{T}, 0, \ldots, 0\right), \frac{\partial f_{23}}{\partial q}=\left(0, . ., 0, \mathcal{M}_{23}\left(q_{2}, q_{3}\right)^{T}, \mathcal{M}_{32}\left(q_{2}, q_{3}\right)^{T}\right)$, and $\frac{\partial f_{30}}{\partial q}=\left(0, \ldots, 0, \mathcal{M}_{30}\left(q_{3}\right)^{T}\right)$. In case of simultaneous collisions (so-called multiple impacts [18, Definition 6.1]), the coefficients of restitution $e_{\mathrm{n}, i j}$ have to satisfy certain conditions to guarantee that the impact law is well-posed $[18, \S 6.2 .4]^{3}$. Notice that if $J_{1}$ is actuated we can use the model depicted in Figure 2 (a) with $\tau_{2}=0$ and control torque $\tau_{1}$, which creates two unilateral contacts which cannot be active at the same time.

When all CoRs $e_{\mathrm{n}, i j}=e_{\mathrm{n}}$, the dynamics in (2) (3) (4) can be rewritten more compactly as a Measure Differential Inclusion (known as Moreau's second order sweeping process [59,36,18]):

$$
M(q) d v+C(q, \dot{q}) \dot{q} d t+g(q) d t-E(q) \tau d t \in-N_{T_{\Phi}(q)}(w(t))
$$

where: $q=\left(q_{1}^{T}, q_{2}^{T}, q_{3}^{T}\right)^{T}, w(t)=\frac{\dot{q}\left(t^{+}\right)+e_{\mathrm{n}} \dot{q}\left(t^{-}\right)}{1+e_{\mathrm{n}}}\left(w(t)\right.$ is equal to $\dot{q}(t)$ outside impacts where $\left.\dot{q}(t)=\dot{q}\left(t^{-}\right)\right)$, $d t$ is the Lebesgue measure, $d v$ is the acceleration measure (i.e., outside impacts $d v=\ddot{q}(t) d t$, at impact times $\left.d v=\left(\dot{q}\left(t^{+}\right)-\dot{q}\left(t^{-}\right)\right) \delta_{t}\right)$. The right-hand side of (5) is the normal cone to the tangent cone $T_{\Phi}(q)$ to the admissible domain $\Phi=\left\{q \in \mathbb{R}^{15} \mid f(q) \geqslant 0\right\}$, see [18, §5.2] for details about its calculation. From (1) without bilateral constraints, we infer that $\nabla f(q) \lambda_{\mathrm{n}, u} \in-N_{T_{\Phi}(q)}(w(t)) \subseteq-N_{\Phi}(q(t))[18, \S \mathrm{B} .2 .2]$. When $q \in \operatorname{int}(\Phi)$, then $T_{\Phi}(q)=\mathbb{R}^{n}$ and $N_{T_{\Phi}(q)}(\cdot)=\{0\}$, when $w \in \operatorname{int}\left(T_{\Phi}(q)\right)$, then $N_{T_{\Phi}(q)}(w)=\{0\}$. At an impact time (5) boils down to $M(q(t))\left[\dot{q}\left(t^{+}\right)-\dot{q}\left(t^{-}\right)\right] \in-N_{T_{\Phi}(q)}(w(t))$ which is equivalent to (4) when $e_{\mathrm{n}, i j}=e_{\mathrm{n}}$ (a global coefficient of restitution) [36] $[18, \S 5.2 .2 .5]$. Several equivalent ways of writing Moreau's impact law exist [18, p.270], one of which is:

$$
\dot{q}\left(t^{+}\right)=-e_{\mathrm{n}} \dot{q}\left(t^{-}\right)+\left(1+e_{\mathrm{n}}\right) \operatorname{proj}_{M(q(t))}\left[T_{\Phi}(q(t)) ; \dot{q}\left(t^{-}\right)\right]
$$

where $\operatorname{proj}_{M(q(t))}$ is the projection in the kinetic matrix metric. This law has limited prediction capabilities for certain multiple (simultaneous) impacts [61, Chapter 3], and can be improved while remaining in a kinematic setting, to allow for different coefficients of restitution at different impact points, and also tangential effects [17]. The advantage of Moreau's impact law is that it is kinematically, kinetically and energetically consistent for all $e_{\mathrm{n}} \in[0,1][36]$.

Remark 1 Assume that there is also a clearance at joint $J_{3}$. One solution to recover a canonical dynamics with $n_{\text {ind }}$ independent subsystems is to add a multiplier associated with this new unilateral constraint, in the right-hand side of the $q_{1}$-dynamics in (2), as $\mathcal{M}_{11}\left(q_{1}\right) \lambda_{\mathrm{n}, u, 11}$. Then add a bilateral holonomic constraint representing joint $J_{2}$ and add one subsystem with dimension three to dynamics in (2).

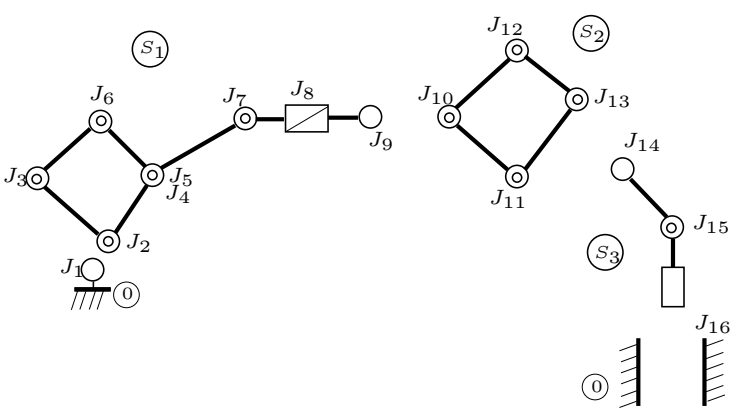

Fig. 3: A kinematic chain with clearances (open system).

\footnotetext{
${ }^{3}$ Let us recall that the CoRs can be chosen non-constant, e.g. velocity dependent, to improve the model's capabilities.
} 
Example 2 Consider now the six-bar system in Figure 5 (a). Without clearance it has three DoFs, one can choose the set of independent coordinates $q_{w c}=\left(\theta_{1}, \theta_{2}, \theta_{3}\right)^{T}$. If a clearance is modeled in joint $J_{3}$ the open system $\mathcal{S}_{1}$ has five DoFs and is made of two subsystems $S_{1}=\left(\mathbf{B}_{1}, \mathbf{B}_{2}\right), S_{2}=\left(\mathbf{B}_{3}, \mathbf{B}_{4}, \mathbf{B}_{5}\right)$, $n_{\text {ind }}=2$. If a clearance is present at both joints $J_{3}$ and $J_{4}$ respectively, the open system $\mathcal{S}_{2}$ has seven DoFs and is made of the three subsystems $S_{1}=\left(\mathbf{B}_{1}, \mathbf{B}_{2}\right), S_{2}=\left(\mathbf{B}_{3}\right), S_{3}=\left(\mathbf{B}_{4}, \mathbf{B}_{5}\right), n_{\text {ind }}=3$. If clearance is present at joints $J_{2}$ and $J_{4}$, then the open system $\mathcal{S}_{3}$ consists of $S_{1}=\left(\mathbf{B}_{1}\right), S_{2}=\left(\mathbf{B}_{2}, \mathbf{B}_{3}\right)$, $S_{3}=\left(\mathbf{B}_{4}, \mathbf{B}_{5}\right), n_{\text {ind }}=3$. In all cases the system can be written in a similar way as (2) (3) (4) with two or three subsystems, respectively. However these three systems may possess different control capabilities. If both $S_{1}$ and $S_{2}$ are fully actuated, $\mathcal{S}_{1}$ is similar to a fully actuated system with one unilateral constraint. On the contrary in $\mathcal{S}_{2}$ the subsystem $S_{2}$ is always unactuated if one considers actuators mounted at the clearance-free joints only. In $\mathcal{S}_{3}$, subsystem $S_{2}$ will be underactuated if only $J_{3}$ has an actuator, with unactuated center of gravity dynamics. Both $\mathcal{S}_{2}$ and $\mathcal{S}_{3}$ possess the structure of so-called juggling systems [22], where a controlled robot controls an uncontrolled (or underactuated) object, see section 4 .

\subsection{Relation with the clearance-free system}

Let us now consider the system in Figure 3 without clearance. Its configuration space $\mathcal{C}_{w c}$ has dimension seven, and it can be described by a vector of independent generalized coordinates ${ }^{4} q_{w c} \in \mathbb{R}^{7}$ :

$$
M_{w c}\left(q_{w c}\right) \ddot{q}_{w c}+C_{w c}\left(q_{w c}, \dot{q}_{w c}\right) \dot{q}_{w c}+g_{w c}\left(q_{w c}\right)=E_{w c}\left(q_{w c}\right) \tau
$$

with possible bilateral holonomic constraints $h_{w c}\left(q_{w c}\right)=0$ if one does not want to work with a set of minimal coordinates, i.e. $q_{w c} \in \mathbb{R}^{n_{w c}}$ with $n_{w c} \geqslant 8$. The relationships between the complementarity Lagrangian system (2) (3) (4) and the clearance-free system (7), are important for the analysis of feedback controllers. First it is necessary to clarify the link between the bilateral constraints imposed by a clearance-free joint, and the unilateral constraints associated with the same joint. Each planar revolute (resp. prismatic) joint gives rise to one (resp. four) unilateral constraint, and in the limit when clearances vanish, both types of joints create two bilateral holonomic constraints. Therefore the clearance-free system is as in (1) with $m_{u}=0$ and $m_{b}$ is augmented to $m_{b}+2 n_{r e v}+2 n_{p r}$ holonomic constraints. If all the bilateral constraints are eliminated, then $\operatorname{dim}\left(q_{w c}\right)=n-m_{b}-2 n_{r e v}-2 n_{p r}$. Consider the system in Example 1, and assume that after reduction $q_{w c}=q_{1}$. Then $M_{1}\left(q_{1}\right) \neq M_{w c}\left(q_{w c}\right)$, because the coordinate reduction implies a redistribution of inertial terms, as shown below. This is important in control problems. Let us apply the coordinate partitioning method in [54] to (1) with $m_{u}=0$ (no clearance), and we set $m_{b}=0$ to simplify the presentation. Setting the clearances to zero gives rise to a set of holonomic constraints $g(q)=0, g: \mathbb{R}^{n} \rightarrow \mathbb{R}^{2 n_{r e v}+2 n_{p r}}$. Assume that there exists a function $\Omega: \mathbb{R}^{n-2 n_{r e v}-2 n_{p r}} \rightarrow \mathbb{R}^{2 n_{r e v}+2 n_{p r}}$ such that $g\left(\Omega\left(q_{w c}\right), q_{w c}\right)=0$ for all $q_{w c} \in \mathbb{R}^{n-2 n_{r e v}-2 n_{p r}}$. Let us partition $q$ as $q=\left(\begin{array}{c}\bar{q} \\ q_{w c}\end{array}\right)$, then on the submanifold $\Sigma_{b} \triangleq\left\{(q, \dot{q}) \mid g(q)=0, \nabla g(q)^{T} \dot{q}=0\right\}$ one has $\bar{q}-\Omega\left(q_{w c}\right)=0$. A nonlinear transformation is defined as $z=Z(q)=\left(\begin{array}{c}\bar{q}-\Omega\left(q_{w c}\right) \\ q_{w c}\end{array}\right)=\left(\begin{array}{c}z_{1} \\ z_{2}\end{array}\right)$, with Jacobian matrix $T(z)=\left(\begin{array}{cc}I_{2 n_{\text {rev }}+2 n_{p r}} & \frac{\partial \Omega}{\partial z_{2}} \\ 0 & I_{n-2 n_{r e v}-2 n_{p r}}\end{array}\right)$. The inverse transformation is $q=Q(z)=\left(\begin{array}{c}z_{1}+\Omega\left(z_{2}\right) \\ z_{2}\end{array}\right)$. Let $V_{2}=\left(0_{\left(n-2\left(n_{r e v}+n_{p r}\right)\right) \times 2\left(n_{r e v}+n_{p r}\right)}, I_{\left(n-2\left(n_{r e v}+n_{p r}\right)\right) \times\left(n-2\left(n_{r e v}+n_{p r}\right)\right)}\right) \in \mathbb{R}^{\left(n-2\left(n_{r e v}+n_{p r}\right)\right) \times n}$. In the coordinates $z$ one obtains an algebraic equation for the Lagrange multiplier associated with the constraints $g(q)=0$, and an unconstrained dynamics as in (7) with $M_{w c}\left(q_{w c}\right)=V_{2} T\left(q_{w c}\right)^{T} M(Q(z)) T\left(q_{w c}\right) V_{2}^{T}$ and $E_{w c}\left(q_{w c}\right)=V_{2} T\left(q_{w c}\right)^{T} E(Q(z))$ (remind that $z_{1}=0$ on $\Sigma_{b}$ ). We see that there is no reason in general that $M_{w c}\left(q_{w c}\right)$ be block diagonal as is $M(q)$. Consider Example 1, with reordering of the coordinates such that $\bar{q}=\left(\begin{array}{c}q_{2} \\ q_{3}\end{array}\right)$ and $q_{w c}=q_{1}$. Calculations yield $M_{w c}\left(q_{w c}\right)=M_{1}\left(q_{w c}\right)+{\frac{\partial \Omega}{\partial z_{2}}}^{T}\left(\begin{array}{cc}M_{2}\left(q_{2}\right) & 0 \\ 0 & M_{3}\left(q_{3}\right)\end{array}\right) \frac{\partial \Omega}{\partial z_{2}}$. A reasonable assumption is the following:

\footnotetext{
4 In general this can be obtained following for instance [54] or [60, §III], see also [4] for a four-bar mechanism.
} 
Assumption 1 The clearance-free system with generalized coordinate $q_{w c} \in \mathbb{R}^{n-m_{b}-2 n_{r e v}-2 n_{p r}}$ is fully or over-actuated, i.e. if $\tau(t) \in \mathbb{R}^{p}$ then $p \geqslant n-m_{b}-2 n_{r e v}-2 n_{p r}$, and $\operatorname{rank}\left(E_{w c}\left(q_{w c}\right)\right)=n-m_{b}-2 n_{r e v}-2 n_{p r}$ for all $q_{w c} \in \mathcal{C}_{w c}$.

For the six-bar system in Figure 5 (a) this means that $p \geqslant 3$, for the four-bar system in Figure 4 (b) this means that $p \geqslant 1$. The degree of actuation is defined in a similar way in $[60, \S I V]$. As alluded to above, clearances introduce severe perturbation in the closed-loop system because of 1) impacts, 2) added degrees of freedom and 3) changing dimensions along system's trajectories. In particular, item 2) renders the fully-actuated clearance-free system, underactuated (with more DoFs than actuators, i.e., $p<n-m_{b}$ in $(1))$.

\section{Feedback control with persistent contact}

The control objective in this section is to apply some "pre-loading" to the system, in order to maintain a persistent contact at all joints with clearance. In other words, to guarantee that $f_{i}(q(t))=0$ for all $t \geqslant 0$ and some $i \in\left\{1, \ldots, m_{u}\right\}$, then analyse how the system may be controlled. We assume that $m_{u}^{\prime} \leqslant m_{u}$ unilateral constraints are active (having $m_{u}=m_{u}^{\prime}$ is obviously not possible if prismatic joints are considered), and we suppose that the vector $f^{\prime}(q) \in \mathbb{R}^{m_{u}^{\prime}}$ gathers all active unilateral contacts. Notice that if there are no clearances then $f_{i}(q)=0$ for all $i \in\left\{1, m_{u}\right\}$, however the converse is false. We first write the so-called contact Linear Complementarity Problem (contact LCP) [18, Chapter 5] [11,72] from (1) at time $t$, where $f^{\prime}(q(t))=0$ and $\nabla f^{\prime}(q(t))^{T} \dot{q}(t)=0$, which is equal in case there are no bilateral constraints to:

$$
0 \leqslant \lambda_{\mathrm{n}, u}^{\prime}(t) \perp \ddot{f}^{\prime}(q(t))=D(q(t)) \lambda_{\mathrm{n}, u}^{\prime}(t)+w(q(t), \dot{q}(t), \tau(t)) \geqslant 0,
$$

where $\lambda_{\mathrm{n}, u}^{\prime}(t) \in \mathbb{R}^{m_{u}^{\prime}}$ is the Lagrange multiplier associated with the active unilateral constraints, $w(q, \dot{q}, \tau)=$ $\frac{d}{d t}\left(\nabla f^{\prime}(q)^{T}\right) \dot{q}+\nabla f^{\prime}(q)^{T} M(q)^{-1}[E(q) \tau-C(q, \dot{q}) \dot{q}-g(q)]$, and $D(q)=\nabla f^{\prime}(q)^{T} M(q)^{-1} \nabla f^{\prime}(q) \in \mathbb{R}^{m_{u}^{\prime} \times m_{u}^{\prime}}$ is the so-called Delassus' matrix. In case independent bilateral constraints are present, a similar LCP holds with a distorted matrix

$$
D_{c}(q)=\nabla f^{\prime}(q)^{T} M(q)^{-1}\left[I_{n}-G(q) M(q)^{-1}\right] \nabla f^{\prime}(q)
$$

$G(q)=\nabla h(q)\left[\nabla h(q)^{T} M(q)^{-1} \nabla h(q)\right]^{-1} \nabla h(q)^{T}[16]$, which is positive definite if and only if all constraints (bilateral and active unilateral) are independent [18, Proposition 5.9], see [16, Proposition 10] for another proof. Let $\mathcal{C}$ denote the $n$-dimensional configuration space of the unconstrained system in (1).

Assumption 2 The bilateral constraints and the active unilateral constraints are independent for all $q \in \mathcal{C} \cap\left\{q \in \mathbb{R}^{n} \mid h(q)=0\right\}$. Moreover if there are $m_{u}^{\prime}$ active unilateral constraints, then $m_{b}+m_{u}^{\prime}<n$.

This assumption guarantees that the contact LCP always has a unique solution, and that the system keeps some DoFs when in contact phase. To simplify the presentation let us assume that there are no bilateral constraints, i.e. $m_{b}=0$. Let the initial data satisfy $f^{\prime}(q(0))=0$ and $\nabla f^{\prime}(q(0))^{T} \dot{q}(0)=$ 0 . Then contact persists if and only if $\ddot{f}^{\prime}(q(t))=0$ for all $t \geqslant 0$, which is equivalent to $\lambda_{\mathrm{n}, u}^{\prime}(t)=$ $-D(q(t))^{-1} w(q(t), \dot{q}(t), \tau(t)) \geqslant 0{ }^{5}$. Let us define

$$
\left\{\begin{array}{l}
\bar{F}(q, \dot{q}) \triangleq \nabla f^{\prime}(q) D(q)^{-1}\left[\frac{d}{d t}\left(\nabla f^{\prime}(q)^{T}\right) \dot{q}-\nabla f^{\prime}(q)^{T} M(q)^{-1}(C(q, \dot{q}) \dot{q}+g(q))\right] \\
\bar{E}(q) \triangleq\left[I_{n}-\nabla f^{\prime}(q) D(q)^{-1} \nabla f^{\prime}(q)^{T} M(q)^{-1}\right] E(q) .
\end{array}\right.
$$

\footnotetext{
${ }^{5}$ However one does not necessarily have $w_{c}(q(t), \dot{q}(t), \tau(t)) \leqslant 0$. All vector inequalities are understood as being componentwise.
} 
The problem of controlling the system with persistent contact at all $m_{u}^{\prime}$ unilateral constraints on $\left[t_{0}, t_{1}\right]$ is given by (we denote $F(q, \dot{q}) \triangleq C(q, \dot{q}) \dot{q}+g(q))$ : Find $\tau(\cdot)$ such that

$$
\left\{\begin{array}{l}
\text { (a) } M(q) \ddot{q}+F(q, \dot{q})+\bar{F}(q, \dot{q})=\bar{E}(q) \tau \\
\text { (b) } f^{\prime}\left(q\left(t_{0}\right)\right)=0, \nabla f^{\prime}\left(q\left(t_{0}\right)\right)^{T} \dot{q}\left(t_{0}\right)=0 \\
(c) 0 \leqslant \lambda_{\mathrm{n}, u}^{\prime} \perp D(q) \lambda_{\mathrm{n}, u}^{\prime}+w(q, \dot{q}, \tau) \geqslant 0 \\
(d) \lambda_{\mathrm{n}, u}^{\prime}=-D(q)^{-1} w(q, \dot{q}, \tau) \geqslant 0 .
\end{array}\right.
$$

The system in (11) has $n-m_{u}^{\prime}$ DoFs. Notice that along the dynamics in (11) (a) we have $\nabla f^{\prime}(q)^{T} \ddot{q}+$ $\frac{d}{d t}\left(\nabla f^{\prime}(q)^{T}\right) \dot{q}=0$, hence the codimension $2 m_{u}^{\prime}$ constraint submanifold defined by $\Sigma_{u}^{\prime} \triangleq\{q \in \mathcal{C}, \dot{q} \in$ $\left.T_{q} \mathcal{C} \mid f^{\prime}(q)=0, \nabla f^{\prime}(q)^{T} \dot{q}=0\right\}$ is invariant under the dynamics (11)(a)(b), where $T_{q} \mathcal{C}$ is the tangent space to $\mathcal{C}$ at $q$. The contact controllability may therefore be stated as:

Definition 1 Let Assumption 2 hold. The system (1) is contact completely controllable in $\Sigma_{u}^{\prime}$ if for any $\left(q_{0}, \dot{q}_{0}\right) \in \Sigma_{u}^{\prime}$ and $\left(q_{1}, \dot{q}_{1}\right) \in \Sigma_{u}^{\prime}$, and any $t_{1} \geqslant t_{0}$, there exists a bounded controller $\tau:\left[t_{0}, t_{1}\right] \rightarrow \mathbb{R}^{p}$ such that the trajectories of $(11)$, which live in $\Sigma_{u}^{\prime}$, satisfy $\left(q\left(t_{0}\right), \dot{q}\left(t_{0}\right)\right)=\left(q_{0}, \dot{q}_{0}\right)$ and $\left(q\left(t_{1}\right), \dot{q}\left(t_{1}\right)\right)=\left(q_{1}, \dot{q}_{1}\right)$.

It appears that (11) (d) implies a constraint on the input $\tau$, such that contact can be maintained via some internal pre-loading within the kinematic chain. If a robust contact mode is sought, then one may write a more stringent inequality as $\lambda_{\mathrm{n}, u}^{\prime}=-D(q)^{-1} w(q, \dot{q}, \tau)=\beta(t)$ for some vector $\mathbb{R}^{m_{u}^{\prime}} \ni \beta(t)>0$, yielding:

$$
D(q)^{-1} \nabla f^{\prime}(q)^{T} M(q)^{-1} E(q) \tau=D(q)^{-1}\left\{-\frac{d}{d t}\left(\nabla f^{\prime}(q)^{T}\right) \dot{q}+\nabla f^{\prime}(q)^{T} M(q)^{-1}[C(q, \dot{q}) \dot{q}+g(q)]\right\}-\beta(t) .
$$

The system (12) is a linear equation with matrix $\hat{E}(q) \triangleq D(q)^{-1} \nabla f^{\prime}(q)^{T} M(q)^{-1} E(q) \in \mathbb{R}^{m_{u}^{\prime} \times p}$. Necessary and sufficient conditions for the existence of $\tau$ that solves (12) for any right-hand side are classical [10, Proposition 6.1.7]. Denoting the right-hand side of $(12)$ as $\hat{w}(q, \dot{q})-\beta(t)$, and $\hat{E}(q)^{\dagger} \in \mathbb{R}^{p \times m_{u}^{\prime}}$ the generalized inverse of $\hat{E}(q)$, we have:

Lemma 1 Let Assumption 2 hold and let an arbitrary vector $\beta(t)>0$ be given. At time $t$, there exists a controller $\tau(t)$ which satisfies (12) if and only if $\left[I_{m_{u}^{\prime}}-\hat{E}(q(t)) \hat{E}(q(t))^{\dagger}\right](\hat{w}(q(t), \dot{q}(t))-\beta(t))=0 \Leftrightarrow$ $\hat{w}(q(t), \dot{q}(t))-\beta(t) \in \operatorname{Im}(\hat{E}(q(t))) \subseteq \mathbb{R}^{m_{u}^{\prime}}$. If existence holds then $\tau(t)=\hat{E}(q(t))^{\dagger}(\hat{w}(q(t), \dot{q}(t))-\beta(t))+z(t)$, with $z(t)=\left(I_{p}-\hat{E}(q(t))^{\dagger} \hat{E}(q(t))\right) \bar{\tau}(t) \in \operatorname{Ker}(\hat{E}(q(t)))$ for some $\bar{\tau}(t) \in \mathbb{R}^{p}$.

The proof follows from [10, Proposition 6.1.7 iv), Proposition 6.1.6]. We see that the rank of the matrix $\nabla f^{\prime}(q)^{T} M(q)^{-1} E(q) \in \mathbb{R}^{m_{u}^{\prime} \times p}$, which reflects the inertial couplings between the input matrix and the constraint gradient, plays a crucial role.

Corollary 1 The conditions of Lemma 1 hold only if $p \geqslant m_{u}^{\prime}$.

Proof Since $\beta(t)$ is chosen arbitrarily, then the conditions hold if and only if $\operatorname{Im}(\hat{E}(q))=\mathbb{R}^{m_{u}^{\prime}} \Leftrightarrow$ $\operatorname{rank}\left(\nabla f^{\prime}(q)^{T} M(q)^{-1} E(q)\right)=m_{u}^{\prime} \Rightarrow p \geqslant m_{u}^{\prime}$.

Therefore the mechanism has to have the same number or more actuators than active unilateral constraints. The second feature of (11) is that the input matrix $E(q)$ is changed to $\bar{E}(q)$ in the system's dynamics (11) (a). We have $\bar{E}(q)=P(q) E(q)$, where the idempotent matrix $P(q)=I_{n}-\bar{P}(q) \in \mathbb{R}^{n \times n}$ is a projector onto $\operatorname{Im}(P(q))=\operatorname{Ker}(\bar{P}(q))$, along $\operatorname{ker}(P(q))=\operatorname{Im}(\bar{P}(q))[46$, §5.8, Theorem 1]. Remind also that $M(q)=\operatorname{blockdiag}\left(M_{i}\left(q_{i}\right)\right), 1 \leqslant i \leqslant n_{\text {ind }}$. Assuming that the conditions of Lemma 1 hold, (11) is a system with holonomic constraints, which is in turn a particular controlled Differential-Algebraic Equation (DAE). In view of the invariance of the constraint submanifold $\Sigma_{u}^{\prime}$ under (11)(a)(b), the controllability of this DAE boils down to the controllability of (11)(a)(b) with control constraint (11)(c)(d).

Assumption 3 The convex polyhedral set $\Omega_{\beta} \triangleq\left\{z \in \mathbb{R}^{p} \mid \hat{E}(q) z \leqslant \hat{w}(q, \dot{q})\right\}$ has a non-empty interior for all $(q, \dot{q}) \in \Sigma_{u}^{\prime}$. 
From Lemma 1 we deduce the system with controller inequality constraint:

$$
\left\{\begin{array}{l}
M(q) \ddot{q}+F(q, \dot{q})+\bar{F}(q, \dot{q})=P(q) E(q)\{\underbrace{\left(I_{p}-\hat{E}(q)^{\dagger} \hat{E}(q)\right) \bar{\tau}}_{\in \operatorname{Ker}(\hat{E}(q))}+\underbrace{\hat{E}(q)^{\dagger} \hat{E}(q) \tau_{\beta}}_{\in \operatorname{Im}\left(\hat{E}(q)^{T}\right)}\}, \\
\text { with : } \\
\quad f^{\prime}(q) \tau_{\beta}-\hat{w}(q, \dot{q})<0 \\
\quad(0))=0, \nabla f^{\prime}(q(0))^{T} \dot{q}(0)=0,
\end{array}\right.
$$

where we could equivalently impose $(q(t), \dot{q}(t)) \in \Sigma_{u}^{\prime}$ for all $t \geqslant 0$. Notice that we could also impose the slightly more stringent condition $\hat{E}(q) \tau_{\beta}-\hat{w}(q, \dot{q})=-\beta(t)$ for some desired contact multiplier $\lambda_{\mathrm{n}, u}^{\prime}(t)=$ $\beta(t)>0$. In this case it follows that $\tau_{\beta}=\hat{E}(q)^{\dagger}\left(\hat{w}(q, \dot{q})-\beta(t)+\left(I_{p}-\hat{E}(q)^{\dagger} \hat{E}(q)\right) \tau_{\beta}^{\prime}\right.$ for any $\tau_{\beta}^{\prime} \in \mathbb{R}^{p}$, and:

$$
M(q) \ddot{q}+F(q, \dot{q})+\bar{F}(q, \dot{q})-\bar{E}(q) \hat{E}(q)^{\dagger}[\hat{w}(q, \dot{q})-\beta(t)]=\bar{E}(q)\left(I_{p}-\hat{E}(q)^{\dagger} \hat{E}(q)\right) \bar{\tau},
$$

where it was used that $\hat{E}(q)^{\dagger} \hat{E}(q)$ is idempotent [10, Proposition 6.1.6], and that $\hat{E}(q)^{\dagger} \hat{E}(q) \hat{E}(q)^{\dagger}=\hat{E}(q)^{\dagger}$ [10, Equ.(6.1.4)]. It is important to see that the dynamics in (14) renders $\Sigma_{u}^{\prime}$ invariant, and guarantees $\lambda_{\mathrm{n}, u}^{\prime}(t)=\beta(t)$ provided the conditions of Lemma 1 hold. In (13) both parts of the input act in mutually orthogonal subspaces since $\operatorname{Ker}(\hat{E}(q))=\left[\operatorname{Im}\left(\hat{E}(q)^{T}\right)\right]^{\perp}\left[10\right.$, Theorem 2.4.3]. From (13) each subsystem $S_{i}$ of the open system, has the controlled dynamics:

$$
\left\{\begin{aligned}
& M_{i}\left(q_{i}\right) \ddot{q}_{i}+F_{i}\left(q_{i}, \dot{q}_{i}\right)+\bar{F}_{i}(q, \dot{q})=\bar{E}_{i}(q) \tau=P_{n_{i}}(q) E(q) \tau \\
& \text { with }: \tau=\hat{E}(q(t))^{\dagger} \hat{E}(q) \tau_{\beta}+\left(I_{p}-\hat{E}(q(t))^{\dagger} \hat{E}(q(t))\right) \bar{\tau} \\
& \hat{E}(q) \tau_{\beta}-\hat{w}(q, \dot{q})<0
\end{aligned}\right.
$$

and $q_{i} \in \mathbb{R}^{n_{i}}, \sum_{i=1}^{n_{\text {ind }}} n_{i}=n, P_{n_{i}}(q) \in \mathbb{R}^{n_{i} \times n}$ and $P(q)=\left[P_{n_{1}}(q)^{T}, P_{n_{2}}(q)^{T}, \ldots, P_{n_{\text {ind }}}(q)^{T}\right]^{T}$. The same as (14) can be written for each $S_{i}$. The open system with clearances will in most cases be underactuated (for instance the clearance-free kinematic chain in Figure 3 is fully actuated with seven actuators $p=7$, while it has fifteen DoFs with clearances; hence $\operatorname{rank}\left(E_{i}\left(q_{i}\right)\right)<n_{i}$ for some $\left.i \in\{1,2,3\}\right)$. The six-bar system in Figure 5 (a) is fully actuated with $p=3$ while joint clearance implies $n \geqslant 5$. The active constraints involve a redistribution of the control actions among the system's degrees of freedom, through the projector $P(q)$, and the generalized force $\bar{F}(q, \dot{q})$ introduces some couplings between the subsystems. Such couplings are however quite different from those considered in [74] for underactuated systems, which involve off-diagonal terms in the inertia matrix. We notice also that (13) (14) is different from [60, (10)-(13)] which uses a coordinate partitioning method on (1) without unilateral contact. It is also noteworthy that the dynamics in (7) is not equivalent to (13) and (14), because active unilateral constraints do not produce the same kinematic constraints as clearance-free joints.

Example 3 Consider as an example the six-bar system in Figure 5 (a), and the open system $\mathcal{S}_{1}=S_{1} \cup S_{2}$, $S_{1}=\left(\mathbf{B}_{1}, \mathbf{B}_{2}\right), S_{2}=\left(\mathbf{B}_{3}, \mathbf{B}_{4}, \mathbf{B}_{5}\right)$, with actuators at joints $J_{1}, J_{2}, J_{4}, J_{5}, J_{6}: m_{u}=1$ (here $m_{u}=m_{u}^{\prime}$ and $\left.f^{\prime}(q)=f(q)\right), p=5, n=5, m_{b}=0,0<D(q) \in \mathbb{R}, E_{1}\left(q_{1}\right)=E_{1}=\left(I_{2}, 0\right) \in \mathbb{R}^{2 \times 5}, E_{2}\left(q_{2}\right)=E_{2}=$ $\left(0, I_{3}\right) \in \mathbb{R}^{3 \times 5}, P(q)=\left(I_{5}-\frac{1}{D(q)} \nabla f(q) \nabla f(q)^{T} M(q)^{-1}\right) \in \mathbb{R}^{5 \times 5}, \hat{E}(q)=\frac{1}{D(q)} \nabla f(q)^{T} M(q)^{-1} \in \mathbb{R}^{1 \times 5}$, $\bar{E}(q)=I_{5}-\frac{1}{D(q)} \nabla f(q) \nabla f(q)^{T} M(q)^{-1}$. The clearance-free system has three DoFs $\left(i . e \cdot \operatorname{dim}\left(q_{w c}\right)=3\right.$ in (7)) and is thus overactuated. The control constraint boils down to $\nabla f(q)^{T} M(q)^{-1} \tau_{\beta}-D(q) \hat{w}(q, \dot{q})<0$ so Assumption 3 holds since $D(q) \hat{w}(q, \dot{q})$ is scalar while $\tau_{\beta} \in \mathbb{R}^{5}$. Calculations yield ${ }^{6}$ :

$$
\begin{aligned}
\bar{F}(q, \dot{q})= & \frac{1}{D(q)}\left(\begin{array}{c}
\nabla_{q_{1}} f(q) \\
\nabla_{q_{2}} f(q)
\end{array}\right)\left\{\frac{d}{d t}\left(\nabla_{q_{1}} f(q)^{T}\right) \dot{q}_{1}+\frac{d}{d t}\left(\nabla_{q_{2}} f(q)^{T}\right) \dot{q}_{2}-\nabla_{q_{1}} f(q) M_{1}\left(q_{1}\right)^{-1} F_{1}\left(q_{1}, \dot{q}_{1}\right)\right. \\
& \left.-\nabla_{q_{2}} f(q) M_{2}\left(q_{2}\right)^{-1} F_{2}\left(q_{2}, \dot{q}_{2}\right)\right\}
\end{aligned}
$$

${ }^{6}$ We employ the notation $\nabla_{q_{i}} f(q)=\frac{\partial f}{\partial q_{i}}(q)^{T}$. 
and

$$
\bar{E}(q)=P(q) E=\left(\begin{array}{l}
E_{1}-\frac{1}{D(q)} \nabla_{q_{1}} f(q) \nabla_{q_{1}} f(q)^{T} M_{1}\left(q_{1}\right)^{-1} E_{1}-\frac{1}{D(q)} \nabla_{q_{1}} f(q) \nabla_{q_{2}} f(q)^{T} M_{2}\left(q_{2}\right)^{-1} E_{2} \\
E_{2}-\frac{1}{D(q)} \nabla_{q_{2}} f(q) \nabla_{q_{1}} f(q)^{T} M_{1}\left(q_{1}\right)^{-1} E_{1}-\frac{1}{D(q)} \nabla_{q_{2}} f(q) \nabla_{q_{2}} f(q)^{T} M_{2}\left(q_{2}\right)^{-1} E_{2}
\end{array}\right)
$$

where the couplings between $S_{1}$ and $S_{2}$ and the input redistribution clearly appear in (16) and (17), respectively.

Example 4 Let us consider the one-DoF prismatic joint in Figure 2. We have $p=2, n=2, m_{u}=$ $2, m_{b}=0, f_{1}(q)=q_{2}-q_{1}-l \geqslant 0, f_{2}(q)=q_{1}-q_{2}+L-l \geqslant 0, \bar{F}(q, \dot{q})=0, \hat{w}(q, \dot{q})=0$. We assume that $f_{1}(q(0))=0$ and $\nabla f_{1}(q(0))^{T} \dot{q}(0)=0$ (i.e. $f^{\prime}(q)=f_{1}(q)$ and $m_{u}^{\prime}=1$ so the necessary condition of Corollary 1 holds), then $\hat{E}=\frac{1}{m_{1}+m_{2}}\left(-m_{2}, m_{1}\right), \hat{E} \tau_{\beta}-\hat{w}=\frac{m_{1} m_{2}}{m_{1}+m_{2}}\left(-\frac{\tau_{\beta_{1}}}{m_{1}}+\frac{\tau_{\beta_{2}}}{m_{2}}\right)$, so that $\hat{E} \tau_{\beta}-\hat{w}<0 \Leftrightarrow \tau_{\beta_{1}}>\frac{m_{1}}{m_{2}} \tau_{\beta_{2}}$, and $\lambda_{\mathrm{n}, u, 1}=\beta>0 \Leftrightarrow \tau_{\beta_{2}}=\frac{m_{2}}{m_{1}} \tau_{\beta_{1}}-\frac{m_{1}+m_{2}}{m_{1}} \beta, \hat{E}^{\dagger}=\frac{m_{1}+m_{2}}{m_{1}^{2}+m_{2}^{2}}\left(\begin{array}{c}-m_{2} \\ m_{1}\end{array}\right)$, $\tau=\left(I_{2}-\frac{1}{m_{1}^{2}+m_{2}^{2}}\left(\begin{array}{cc}m_{2}^{2} & -m_{1} m_{2} \\ -m_{1} m_{2} & m_{1}^{2}\end{array}\right)\right) \bar{\tau}+\left(\begin{array}{c}\frac{m_{2}\left(m_{1}+m_{2}\right)}{m_{1}^{2}+m_{2}^{2}} \beta(t) \\ \frac{-m_{1}\left(m_{1}+m_{2}\right)}{m_{1}^{2}+m_{2}^{2}} \beta(t)\end{array}\right)$. The persistent contact dynamics is given by:

$$
\left\{\begin{array}{l}
m_{1} \ddot{q}_{1}-\frac{m_{2}\left(m_{1}+m_{2}\right)}{m_{1}^{2}+m_{2}^{2}} \beta(t)=\bar{\tau}_{1}-\frac{1}{m_{1}^{2}+m_{2}^{2}}\left(m_{2}^{2} \bar{\tau}_{1}-m_{1} m_{2} \bar{\tau}_{2}\right) \\
m_{2} \ddot{q}_{2}+\frac{m_{1}\left(m_{1}+m_{2}\right)}{m_{1}^{2}+m_{2}^{2}} \beta(t)=\bar{\tau}_{2}-\frac{1}{m_{1}^{2}+m_{2}^{2}}\left(-m_{1} m_{2} \bar{\tau}_{1}+m_{1}^{2} \bar{\tau}_{2}\right) \\
q_{2}(0)-q_{1}(0)=l, \dot{q}_{2}(0)=\dot{q}_{1}(0), \beta(t)>0 .
\end{array}\right.
$$

which is $(14)$, or

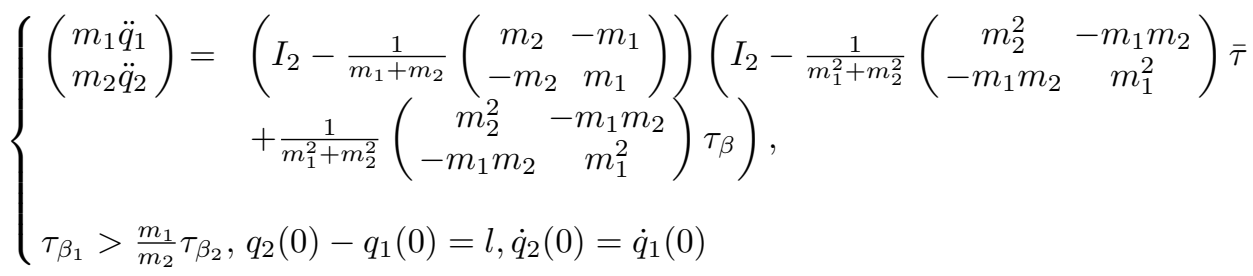

which is $(13)$.

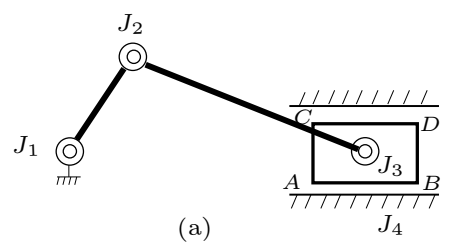

Fig. 4: (a) Slider-crank, and (b) four-bar systems.

Example 5 Consider the gear system in Figure 2 (c), which is a simple example of redundant-drive mechanism [26] which are designed in many mechanisms like engines [72, Chapter 11], and is made of three subsystems (each cogwheel). We have $q=\left(\theta_{1}, \theta_{2}, \theta_{3}\right)^{T}, n=3, m_{u}=4, m_{b}=0, p=3, m_{u}^{\prime}=0$, or 1 or 2. The four unilateral constraints are $f_{1}(q)=-\theta_{1}+\theta_{2}+\alpha_{1} \geqslant 0$ (unilateral contact between teeth $T_{21}$ and $\left.T_{11}\right), f_{2}(q)=\theta_{1}-\theta_{2}+\alpha_{2} \geqslant 0$ (unilateral contact between teeth $T_{22}$ and $T_{11}$ ), $f_{3}(q)=-\theta_{3}+\theta_{2}+\alpha_{3} \geqslant 0$ (unilateral contact between teeth $T_{23}$ and $T_{31}$ ), $f_{4}(q)=-\theta_{3}-\theta_{2}+\alpha_{4} \geqslant 0$ (unilateral contact between 
teeth $T_{24}$ and $T_{31}$ ), for some $\alpha_{i}, 1 \leqslant i \leqslant 4^{7}$. Assume that $f_{1}(q)=0$ and $f_{4}(q)=0$ (persistent contact between teeth $T_{21}$ and $T_{11}$, and teeth $T_{31}$ and $\left.T_{24}\right)$. This yields $D(q)=D=\left(\begin{array}{cc}\frac{J_{1}+J_{2}}{J_{1} J_{2}} & -\frac{1}{J_{2}} \\ -\frac{1}{J_{2}} & \frac{J_{3}+J_{2}}{J_{3} J_{2}}\end{array}\right) \succ 0$, where $J_{i}$ is the inertia of cogwheel $i$. Let $J_{1}=J_{2}=J_{3}=J \mathrm{~kg} \cdot \mathrm{m}^{2}$, then $\hat{E}=\frac{1}{3}\left(\begin{array}{ccc}-2 & 1 & 1 \\ -1 & -1 & 2\end{array}\right)^{8}$. We have $\hat{w}(q, \dot{q})=0$ (we could easily add torques like viscous friction), thus the persistent contact condition is $\hat{E} \tau_{\beta}<0$, equivalently $2 \tau_{\beta_{1}}>\tau_{\beta_{2}}+\tau_{\beta_{3}}$ and $\tau_{\beta_{1}}>-\tau_{\beta_{2}}+2 \tau_{\beta_{3}}$, which implies $\tau_{\beta_{1}}>\tau_{\beta_{3}}$. This study could be extended to the family of redundant-drive systems analysed in [26].

Example 6 The benchmark slider-crank and four-bar systems in Figure 4 both have $\operatorname{dim}\left(q_{w c}\right)=1$, so full actuation of the clearance-free systems means $p=1$. Consider the slider-crank with mechanical play in the prismatic joint $J_{4}$, so that $n=3, m_{u}=4$. A control objective may be to maintain persistent contact at corners $A$ and $B$, or at $C$ and $D$. Then $m_{u}^{\prime}=2$ and Corollary 1 implies $p \geqslant 2$, hence overactuation of the clearance-free system (remember this is just a necessary condition). Consider the four-bar system, with a clearance at joint $J_{4}$, so that $n=3, m_{u}=1$. From Corollary 1 it is necessary that $p \geqslant 1$ so overactuation is not a priori necessary. Obviously in both cases checking whether the conditions of Lemma 1 hold, requires calculations outside the scope of this article.

Further developments on the persistent contact control design are in Remark 3 below.

\subsection{Contact null-controllability (all linear case)}

Let us now analyse the controllability of (13) in the all-linear case: $f^{\prime}(q)=F q+G \geqslant 0, M(q)=M$, $g(q)=K q, E(q)=E$. Let $x=\left(q^{T}, \dot{q}^{T}\right)^{T}$. The system in (13) becomes:

$$
\dot{x}(t)=A x(t)+B u(t), \quad \hat{E} u_{2}(t)-F M^{-1} K x_{1}(t)<0,
$$

for all $t \geqslant 0$, with $A=\left(\begin{array}{cc}I_{n} \\ -M^{-1}\left(K+F^{T} D^{-1} K\right) & 0\end{array}\right) \in \mathbb{R}^{2 n \times 2 n}, B=\left(\begin{array}{cc}0 & 0 \\ P E\left(I_{p}-\hat{E}^{\dagger} \hat{E}\right) P E \hat{E}^{\dagger} \hat{E}\end{array}\right) \in$ $\mathbb{R}^{2 n \times 2 p}, u=\left(\begin{array}{c}u_{1} \\ u_{2}\end{array}\right)=\left(\begin{array}{c}\bar{\tau} \\ \tau_{\beta}\end{array}\right) \in \mathbb{R}^{2 p}$, and initial data $F x_{1}(0)+G=0, F x_{2}(0)=0\left(\Rightarrow F x_{1}(t)+G=0\right.$ and $F x_{2}(t)=0$ for all $\left.t \geqslant 0\right)$. The null-controllability of linear invariant systems with constrained inputs has been studied in $[13,76]$. We remind that null-controllability means that the system's state can be steered to the origin in finite time from any initial condition, with an admissible control.

Proposition 1 Let Assumptions 2 and 3 hold. Let $\Omega_{\beta}=\left\{u_{2} \in \mathbb{R}^{p} \mid \hat{E} u_{2}-F M^{-1} K x_{1}<0\right.$ for all F $x_{1}+$ $G=0\}$. (i) Suppose that $0 \in \Omega_{\beta}$, then the system (20) is null-controllable if and only if rank $(A, B)=2 n$.

(ii) Suppose that there exists $u_{2} \in \Omega_{\beta}$ such that $P E \hat{E}^{T} E u_{2}=0$, and the interior of $\Omega_{\beta} \neq \emptyset$ in $\mathbb{R}^{p}$. Then null-controllability of (20) holds if and only if a) $\operatorname{rank}(K(A, B))=2 n^{9}$, and b) there is no real eigenvector $v=\left(v_{1}^{T}, v_{2}^{T}\right)^{T} \in \mathbb{R}^{p} \times \mathbb{R}^{p}$ of $A^{T}$ such that $v_{2}^{T}\left[P E\left(I_{p}-\hat{E}^{\dagger} \hat{E}\right) u_{1}+\hat{E}^{\dagger} \hat{E} u_{2}\right] \leqslant 0$ for all $u \in \mathbb{R}^{p} \times \Omega_{\beta}$.

Proof The proof of (i) follows from [13, Theorem 1.1], that of (ii) follows from [13, Theorem 1.4].

Proposition 1 is interesting because it shows that the conditions that guarantee the contact controllability of Definition 1, may be hard to attain for practical mechanisms. Necessary and sufficient conditions for the global controllablity are given in [13, Corollary 3.4], however the result applies when the set of admissible controllers is a cone with vertex at the origin, which implies here that $\hat{w}(q, \dot{q})=0$. Another result $[13$, Theorem 3.5] [76, Theorem 5.1, Corollary 5.2] concerns bounded inputs and steering the origin to the whole state space $\mathbb{R}^{2 n}$. Then a necessary condition is that $A$ has all its eigenvalues with non negative real parts.

\footnotetext{
7 More realistic gap functions would be with $\alpha_{i}=\alpha_{i}\left(\theta_{2}\right)$ depending on teeth geometry. We keep the simplest form in this short example.

8 We consider here the control torques as in Figure 2 (c), but other types of actuators may be implemented [38] that could yield different input matrix $E(q)$.

$9 K(A, B)$ denotes the Kalman controllability matrix of the pair $(A, B)$.
} 


\subsection{Complete contact controllability}

One path is to analyze the controllability using existing criteria for systems with positive inputs [77]. However most of systems of interest in practice are nonlinear, for which null-controllability may be stated locally only, around the origin [77, Corollary 6.3]. The results in [48] may be used to analyse the local configuration controllability of mechanical systems without control constraint. They may be used to state a necessary condition for the local configuration controllability of (13) or (14). Consider (18), and let us multiply both sides on the left by $\left(m_{1}, m_{2}\right) \in \mathbb{R}^{1 \times 2}$. This results in $m_{1}^{2} \ddot{q}_{1}+m_{2}^{2} \ddot{q}_{2}=m_{1} \bar{\tau}_{1}+m_{2} \bar{\tau}_{2}$, i.e. we have cancelled out the terms in $\beta(t)$, equivalently $\left(m_{1}, m_{2}\right)^{T} \in \operatorname{Ker}\left[\left(\bar{E}(q) \hat{E}(q)^{\dagger}\right)^{T}\right]$ in $(14)$. Furthermore since trajectories live in $\Sigma_{u}^{\prime}$, we have for all $t \geqslant 0: f_{1}(q(t))=0 \Leftrightarrow q_{1}(t)+l=q_{2}(t), \dot{q}_{1}(t)=\dot{q}_{2}(t), \ddot{q}_{1}(t)=\ddot{q}_{2}(t)$. Hence the contact dynamics with the controller $\tau$ in Example 4, is the reduced (unconstrained) dynamics $\left(m_{1}^{2}+m_{2}^{2}\right) \ddot{q}(t)=m_{1} \bar{\tau}_{1}(t)+m_{2} \bar{\tau}_{2}(t)$ where $q(\cdot)$ may be chosen as $q_{1}(\cdot)$ or $q_{2}(\cdot)$. It is clear that the reduced dynamics is controllable. We infer that the system is contact completely controllable in the sense of Definition 1, which is intuitively clear for this simple system. This motivates us to state the following:

Proposition 2 Consider the system in (14), and assume that the conditions of Lemma 1 hold $\left(\Rightarrow p \geqslant m_{u}^{\prime}\right.$ from Corollary 1) with some $\beta(t)>0$ and for all $t \geqslant 0$. This system is contact completely controllable in $\Sigma_{u}^{\prime}$ if there exists $A(q) \in \mathbb{R}^{\left(n-m_{u}^{\prime}\right) \times n}$, and a differentiable function $\Omega: \mathbb{R}^{\left(n-m_{u}^{\prime}\right)} \rightarrow \mathbb{R}^{m_{u}^{\prime}}$, such that 1) $q=\left(\begin{array}{l}q_{1} \\ q_{2}\end{array}\right), q_{1} \in \mathbb{R}^{m_{u}^{\prime}}, q_{2} \in \mathbb{R}^{n-m_{u}^{\prime}}, q_{1}=\Omega\left(q_{2}\right)$, 2) $A(q) \bar{E}(q) \hat{E}(q)^{\dagger}=0$ for all $q \in \Sigma_{u}^{\prime}$, and the system with state $\left(q_{2}, \dot{q}_{2}\right)$ :

$$
\mathcal{M}\left(q_{2}\right) \ddot{q}_{2}+\mathcal{F}\left(q_{2}, \dot{q}_{2}\right)=\mathcal{E}\left(q_{2}\right) \bar{\tau}
$$

is completely controllable, where $\mathcal{M}\left(q_{2}\right)=A\left(q_{2}\right) M\left(q_{2}\right)\left(\begin{array}{c}\frac{\partial \Omega}{\partial q_{2}} \\ I_{n-m_{u}^{\prime}}\end{array}\right) \in \mathbb{R}^{\left(n-m_{u}^{\prime}\right) \times\left(n-m_{u}^{\prime}\right)}, \mathcal{E}\left(q_{2}\right)=A\left(q_{2}\right) \bar{E}\left(q_{2}\right) \in$ $\mathbb{R}^{\left(n-m_{u}^{\prime}\right) \times p}, \mathcal{F}\left(q_{2}\right)=A\left(q_{2}\right)\left(F\left(q_{2}, \dot{q}_{2}\right)+\bar{F}\left(q_{2}, \dot{q}_{2}\right)\right)$.

Proof Consider (14), from the second assumption of the proposition we obtain:

$$
A(q) M(q) \ddot{q}+A(q)[F(q, \dot{q})+\bar{F}(q, \dot{q})]=A(q) \bar{E}(q) \bar{\tau}
$$

Using the first assumption, we get $M(q) \ddot{q}=M\left(q_{2}\right)\left(\begin{array}{c}\frac{\partial \Omega}{\partial q_{2}} \\ I_{n-m_{u}^{\prime}}^{\prime}\end{array}\right) \ddot{q}_{2}+\left(\begin{array}{c}M_{11}\left(q_{2}\right) \\ M_{12}\left(q_{2}\right)^{T}\end{array}\right) \frac{d}{d t}\left(\frac{\partial \Omega}{\partial q_{2}}\right) \dot{q}_{2}$, where the argument $q$ can be replaced by $q_{2}$ with some abuse of notation $\left(M(q)=M\left(q_{1}, q_{2}\right)=M\left(\Omega\left(q_{2}\right), q_{2}\right)=\right.$ $M\left(q_{2}\right)$, and so on for the other terms).

The controllability of the nonlinear system in (21) may be studied using various general criteria for strong accessibility, local controllability, etc, and is not tackled here. A similar analysis could be done starting from (13) instead of (14). In general the reduced dynamics in (21) is not a Lagrangian system. It is also noteworthy that using some coordinate partitioning as the McClamroch-Wang's generalized coordinate change [54], to the persistently constrained dynamics with $f^{\prime}(q)=0$, would not yield the same result and would bring little advantage. The reason is that this method decouples the "normal" and "tangent" (independent of $\lambda_{\mathrm{n}, u}^{\prime}$ ) dynamics to the submanifold $\Sigma_{u}$ in a clever way (preserving the Lagrangian structure), however in our case the term $\bar{E}(q) \hat{E}(q)^{\dagger} \beta(t)$ would not be in general cancelled out from the "tangent" dynamics. The controllability of (21) will be influenced by the inputs redistribution and inertial couplings introduced through $\bar{E}(q)$ and $\bar{F}(q, \dot{q})$. An interesting topic is to investigate whether the clearances, which add DoFs and therefore modify the system's kinematics, could allow to improve the capabilities of the clearance-free system when using a persistent contact controller. For example, Watt, Roberts or Chebyshev linkages are known to produce approximate straight lines. Is it possible to get a better approximation adding clearances and persistent contact control ? Further developments on the persistent contact control design are in Remark 3 below. 


\subsection{Including frictional effects}

To simplify the presentation we suppose that there are only unilateral constraints. Friction is introduced in the right-hand side of $(1)$ as $[2,18,35,72]$ :

$$
\left\{\begin{array}{l}
M(q) \ddot{q}+C(q, \dot{q}) \dot{q}+G(q)=E(q) \tau+\nabla f(q) \lambda_{\mathrm{n}, u}+H_{\mathrm{t}, u}(q) \lambda_{\mathrm{t}, u} \\
\left(\lambda_{\mathrm{n}, u}, \lambda_{\mathrm{t}, u}\right) \text { satisfies friction law }
\end{array}\right.
$$

where the matrix $H_{\mathrm{t}, u}(q)$ can be obtained from the local kinematics at each contact point $i$ and applying the virtual work principle, $\lambda_{\mathrm{t}, u}=\left(\lambda_{\mathrm{t}, u, 1}^{T}, \ldots, \lambda_{\mathrm{t}, u, m_{u}}^{T}\right)^{T}, \lambda_{\mathrm{t}, u, i} \in \mathbb{R}^{d}, d=1$ for planar friction, $d=2$ for three-dimensional friction. Let us adopt Coulomb's friction, keeping in mind that varying sliding coefficients could be used (like Stribeck effects [18, Remark 5.16]). The well-posedness issue with friction is analysed in $[67,68]$. Let us examine how the above results extend to the case with sliding tangential motion, i.e. we assume that there are $m_{u}^{\prime}$ sliding unilateral contacts with coefficients of friction $\mu_{i}>0$. We restrict ourselves to the planar case so that $\lambda_{\mathrm{t}, u, i}^{\prime}=-\mu_{i} \lambda_{\mathrm{n}, u, i}^{\prime} \operatorname{sgn}\left(v_{\mathrm{t}, u, i}\right), v_{\mathrm{t}, u}=H_{\mathrm{t}, u}(q)^{T} \dot{q}, 1 \leqslant i \leqslant m_{u}^{\prime}$. Relying on $[11, \S 3]$ or $[18$, Section 5.5], we find that the contact LCP (8) becomes:

$$
0 \leqslant \lambda_{\mathrm{n}, u}^{\prime}(t) \perp D^{\mu}(q(t)) \lambda_{\mathrm{n}, u}^{\prime}(t)+w(q(t), \dot{q}(t), \tau(t)) \geqslant 0
$$

with $w(q(t), \dot{q}(t), \tau(t))=\frac{d}{d t}\left(\nabla f^{\prime}(q)^{T}\right) \dot{q}+\nabla f^{\prime}(q)^{T} M(q)^{-1}[E(q) \tau-C(q, \dot{q}) \dot{q}-g(q)]$ as in $(8)$, and $D^{\mu}(q)=$ $D(q)-\nabla f^{\prime}(q)^{T} M(q)^{-1} H_{\mathrm{t}, u}(q)[\mu]\left[\operatorname{sgn}\left(v_{\mathrm{t}, u, i}\right)\right], 1 \leqslant i \leqslant m_{u}^{\prime}$. Applying [11, Proposition 12] it follows that for small enough friction coefficients, the contact LCP in (24) always has a unique solution and $D^{\mu}(q) \succ 0$ for all $q \in \mathbb{R}^{n}$ (but non symmetric). As above the persistent contact with the $m_{u}^{\prime}$ constraints implies that $\lambda_{\mathrm{n}, u}^{\prime}(t)>0$ for all $t \geqslant 0$, consequently $D^{\mu}(q(t)) \lambda_{\mathrm{n}, u}^{\prime}(t)+w(q(t), \dot{q}(t), \tau(t))=0$, and $\lambda_{\mathrm{n}, u}^{\prime}(t)=$ $-D^{\mu}(q(t))^{-1} w(q(t), \dot{q}(t), \tau(t))$. The remaining part of the analysis copies the above one, replacing $D(q)$ by $D^{\mu}(q)$ in $\left.\bar{F}(q, \dot{q}), \bar{E}(q), \hat{w}(q), \dot{q}\right)$ and $\hat{E}(q)$. We have assumed that all contacts are silding, which may not be satisfied. A complete analysis should take into account possible sticking contacts and stick/slip transitions, or derive conditions which guarantee slipping at all times. The friction model may be Coulomb's law or an extension that keeps the multivalued feature at zero tangential velocity, hence correctly handling sticking phases, a crucial property in multibody dynamics ${ }^{10}$. It is certainly of utmost importance to carefully examine singularities due to Painlevé paradoxes, which may occur even for small friction coefficients [18, 25]. Such frictional singularities may become quite disturbing in some cases like prismatic joints. The tools in [11] could be used to determine upperbounds on friction coefficients under which no paradox occur. In many cases joint lubrication plays a crucial role in tangential effects [49], and specific models should be used. Finally we note that mechanisms with joint clearance are one instance where friction plays a quite different role than in other classes of nonsmooth Lagrangian systems, e.g. biped robots.

\section{Control with impacting trajectories}

While the foregoing section focuses on impact-free trajectories, it is of interest to investigate the control of systems with clearances when collisions inside the joints may occur (one reason being that control with persistent contact is not always possible). More precisely, collisions are seen here as part of the trajectory and control design, not as disturbances.

\footnotetext{
10 Other friction models which are popular in the Control community, like Leuven's [45] may be tried. However such models involve many parameters per contact (usually six or seven) whose physical meaning may not always be clear [86], and whose identification may become quite difficult in case of many contacts with poorly known materials properties. Moreover they yield stiff differential equations around vanishing tangential velocity because of regularization of the sticking mode. The choice of the friction model will depend on the control objectives, number of contacts, temperature variation, accurate knowledge of materials, etc, and it is difficult to assert a priori which model may provide the best results. We take the point of view here to start from the simplest set-valued Coulomb's model, or modifications that preserve set-valuedness at zero tangential velocity, and which are quite efficient in multibody multicontact systems $[2,72]$.
} 
4.1 Periodic trajectories in flows with collision

The basic idea is to take advantage of impact mappings in order to control a system through a suitable sequence of free-motion (all unilateral contacts in clearances are deactivated $f(q)>0$ ) and of impacts between two (or more than two) bodies. Mathematically speaking, the system is a flow with collisions $[18, \S 1.3 .2]$. The first step is to show that some periodic trajectory exists, using for instance a feedforward input (open-loop) applied at some joints, like a periodic excitation. This type of analysis boils down to solving a nonlinear equation $H(x)=0$, where the vector $x$ contains boundary conditions and system's parameters, and is constructed by concatenating solutions between collisions and impact mappings (the number and location of the actuated joints certainly plays a crucial role in the construction of the function $H(\cdot))$. Once the step of the existence of some trajectory is taken, its stability may be analysed with an impact Poincaré map (that is, the Poincaré section is chosen in $\operatorname{bd}(\Phi) \cap V\left(q\left(t_{k}\right)\right)$, where $\Phi$ is the admissible domain of the configuration space, post-impact velocities are in the polyhedral tangent cone to $\Phi$ at $q\left(t_{k}\right): V\left(q\left(t_{k}\right)\right)=\left\{v \in \mathbb{R}^{n} \mid v^{T} \nabla f_{i}\left(q\left(t_{k}\right)\right) \geqslant 0, i \in \mathcal{J}\left(q\left(t_{k}\right)\right)\right\}, \mathcal{J}\left(q\left(t_{k}\right)\right) \subseteq\left\{1, \ldots, m_{u}\right\}$ the index set of active constraints, $t_{k}$ is an impact time. This approach is limited to simple systems where solutions of the unconstrained system are known analytically (like impact oscillators [18, §7.3], see also [53, Lemmae 1 and 2] for existence of locally stable periodic orbits with non-collocated and collocated proportionalderivative controllers, and more references in $[18, \S 7.3 .4, \S 7.3 .6]$ ). It yields in general local stability (with rough estimate of the basins of attractions), and is most of the time analytically untractable. Hence only numerical analysis may be done, which is not quite satisfactory in a feedback control analysis context. We will describe in section 4.2 a path that may be used to simplify the process, after a suitable pre-feedback controller has been designed.

\subsection{Control through impacts (juggling systems)}

Consider the six-bar system in Figure $5(\mathrm{a})$, with clearance at joints $J_{3}$ and $J_{4}$, and actuators at joints $J_{1}, J_{2}, J_{5}, J_{6}$ (thus the clearance-free system is over-actuated since $\operatorname{dim}\left(q_{w c}\right)=3$ and $p=4$ ), as depicted in Figure 5 (a). The system has seven DoFs and is composed of three subsystems $S_{1}=\left(\mathbf{B}_{1}, \mathbf{B}_{2}\right), q_{1}=$ $\left(\theta_{1}, \theta_{2}\right)^{T} \in \mathbb{R}^{2}, S_{2}=\left(\mathbf{B}_{3}\right), q_{2}=\left(x_{3}, y_{3}, \theta_{3}\right)^{T}, S_{3}=\left(\mathbf{B}_{4}, \mathbf{B}_{5}\right), q_{3}=\left(\theta_{4}, \theta_{5}\right)^{T}$. Both $S_{1}$ and $S_{3}$ are fully actuated planar manipulators, while $S_{2}$ is unactuated and evolves autonomously (possibly under the influence of gravity) when the journal and bearing at both joints $J_{3}$ and $J_{4}$ are not in contact. This six-bar system possesses the following dynamics (the contact forces are impulsive at impact times):

$$
\left\{\begin{array}{l}
\text { (a) } M_{2} \ddot{q}_{2}+G_{2}\left(q_{2}\right)=\mathcal{M}_{21}\left(q_{2}, q_{1}\right) \lambda_{\mathrm{n}, u, 12}+\mathcal{M}_{23}\left(q_{2}, q_{3}\right) \lambda_{\mathrm{n}, u, 23} \\
\text { (b) } M_{1}\left(q_{1}\right) \ddot{q}_{1}+F_{1}\left(q_{1}, \dot{q}_{1}\right)=\tau_{1}+\mathcal{M}_{12}\left(q_{1}, q_{2}\right) \lambda_{\mathrm{n}, u, 12} \\
\text { (c) } M_{3}\left(q_{3}\right) \ddot{q}_{3}+F_{3}\left(q_{3}, \dot{q}_{3}\right)=\tau_{3}+\mathcal{M}_{32}\left(q_{2}, q_{3}\right) \lambda_{\mathrm{n}, u, 23} \\
\text { (d) } 0 \leqslant f_{12}\left(q_{1}, q_{2}\right) \perp \lambda_{\mathrm{n}, u, 12} \geqslant 0,0 \leqslant f_{23}\left(q_{2}, q_{3}\right) \perp \lambda_{\mathrm{n}, u, 13} \geqslant 0 \\
\text { (e) Impact law in }(4),
\end{array}\right.
$$

where we adopted the notations of Example 1, ij $=12$ and $23, \tau_{1} \in \mathbb{R}^{2}, \tau_{3} \in \mathbb{R}^{2}, q_{2} \in \mathbb{R}^{3}$ (body $\mathbf{B}_{3}$ center of mass coordinates, plus $\left.\theta_{3}\right)$. We may also consider the six-bar system with clearances at joints $J_{3}, J_{5}$, and actuation at joints $J_{1}, J_{2}, J_{4}$ and $J_{6}$ as depicted in Figure 6 (a). Here $S_{1}=\left(\mathbf{B}_{1}, \mathbf{B}_{2}\right), S_{2}=\left(\mathbf{B}_{3}, \mathbf{B}_{4}\right)$, $S_{3}=\left(\mathbf{B}_{5}\right)$. Subsystem $S_{2}$ has four DoFs, $q_{2} \in \mathbb{R}^{4}$, with degree of underactuation three since $\tau_{2}$ (applied at $\left.J_{4}\right)$ is scalar, $S_{1}$ has two DoFs, $q_{1}=\left(\theta_{1}, \theta_{2}\right)^{T} \in \mathbb{R}^{2}, S_{3}$ has one DoF, $q_{3}=\theta_{5} \in \mathbb{R}$ and they are both fully actuated, with $\tau_{1} \in \mathbb{R}^{2}, \tau_{3} \in \mathbb{R}$. The clearance-free system is overactuated with $p=4$ and $\operatorname{dim}\left(q_{w c}\right)=3$. When airborne (no journal/bearing contact at joints $J_{3}$ and $J_{5}$ ), the dynamics of the center of mass (CoM) of $S_{2}$ is influenced only by external actions and not by the control at $\tau_{2}$ which is an internal torque which works on $\theta_{3}-\theta_{4}$ (hence the input matrix $E_{2}$ ). The unconstrained motion dynamics 
is given by (where we do not repeat the impact law for briefness):

$$
\left\{\begin{array}{l}
\text { (a) } M_{2} \ddot{q}_{2}+G_{2}\left(q_{2}\right)=\mathcal{M}_{21}\left(q_{1}, q_{2}\right) \lambda_{\mathrm{n}, u, 12}+\mathcal{M}_{23}\left(q_{2}, q_{3}\right) \lambda_{\mathrm{n}, u, 23}+E_{2} \tau_{2} \\
\text { (b) } M_{1}\left(q_{1}\right) \ddot{q}_{1}+C\left(q_{1}, \dot{q}_{1}\right) \dot{q}_{1}+G\left(q_{1}\right)=\tau_{1}+\mathcal{M}_{12}\left(q_{1}, q_{2}\right) \lambda_{\mathrm{n}, u, 12} \\
\text { (c) } M_{3} \ddot{q}_{3}+G_{3}\left(q_{3}\right)=\tau_{3}+\mathcal{M}_{32}\left(q_{2}, q_{3}\right) \lambda_{\mathrm{n}, u, 23} \\
\text { (d) } 0 \leqslant f_{12}\left(q_{1}, q_{2}\right) \perp \lambda_{\mathrm{n}, u, 12} \geqslant 0,0 \leqslant f_{23}\left(q_{2}, q_{3}\right) \perp \lambda_{\mathrm{n}, u, 23} \geqslant 0 .
\end{array}\right.
$$

The dynamics of the centre of mass of $S_{2}$ in (26) (a) is in turn given by:

$$
\left(m_{3}+m_{4}\right) \ddot{q}_{c o m}=G_{c o m}\left(q_{2}\right)+\mathcal{M}_{c o m}\left(q_{2}, q_{1}, q_{3}\right)\left(\begin{array}{c}
\lambda_{\mathrm{n}, u, 12} \\
\lambda_{\mathrm{n}, u, 23}
\end{array}\right)
$$

for some vector $G_{\text {com }}\left(q_{2}\right) \in \mathbb{R}^{2}$ and matrix $\mathcal{M}_{\text {com }}\left(q_{2}, q_{1}, q_{3}\right) \in \mathbb{R}^{2 \times 2}$, so we implicitly chose $q_{2}=$ $\left(q_{c o m}^{T}, \theta_{3}, \theta_{4}\right)^{T}$. The other part of the dynamics in (25) (a) has the form:

$$
\left(\begin{array}{c}
I_{3} \ddot{\theta}_{3} \\
I_{4} \ddot{\theta}_{4}
\end{array}\right)=\mathcal{M}_{\theta}\left(q_{2}, q_{1}, q_{3}\right)\left(\begin{array}{c}
\lambda_{\mathrm{n}, u, 12} \\
\lambda_{\mathrm{n}, u, 23}
\end{array}\right)+E_{\theta} \tau_{2},
$$

for some vector matrix $\mathcal{M}_{\theta}\left(q_{2}, q_{1}, q_{3}\right) \in \mathbb{R}^{2 \times 2}$, and $E_{\theta}=\left(\begin{array}{c}1 \\ -1\end{array}\right)$ from the virtual work principle. The analytic integrability of the CoM's dynamics during unconstrained motion depends on $G_{c o m}\left(q_{2}\right)$, similarly to (25) (a). It is clear that the free-motion dynamics is uncontrollable since (25) (a) and (27) are uncontrolled systems when the contact forces vanish (non bearing/journal contact). Both (25) and (26) (27) (28) have therefore the structure of so-called juggling systems [22, Equations (11)-(13)] (the systems depicted in Figure 2 (a) (b) (c) with $\tau_{2}=0$ also belong to this class).

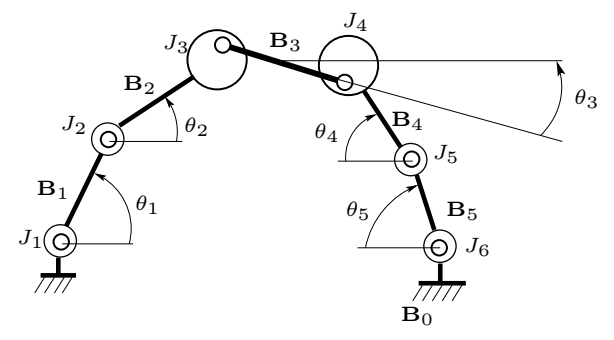

(a)

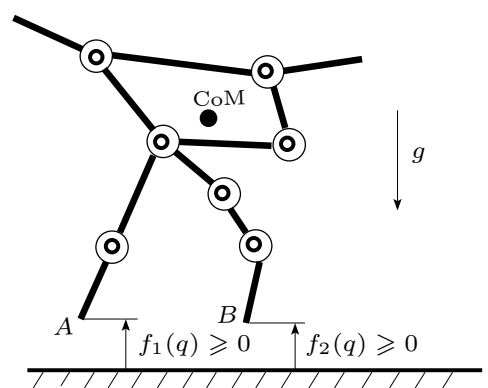

(b)

Fig. 5: (a) Six-bar mechanism with clearances at $J_{3}, J_{4}$; (b) Biped robot.

Remark 2 (The class of juggling controlled systems) Jugglings systems have the general dynamics [22]:

$$
\left\{\begin{array}{l}
(a) \dot{z}_{1}+F_{1}\left(z_{1}, t\right)=\mathcal{M}_{12}\left(z_{1}, z_{2}\right) \lambda_{12} \\
(b) \dot{z}_{2}+F_{2}\left(z_{1}, z_{2}, t\right)=\mathcal{M}_{21}\left(z_{1}, z_{2}\right) \lambda_{12}+E\left(z_{2}\right) \tau \\
0 \leqslant f\left(z_{1}, z_{2}\right) \perp \lambda_{12} \geqslant 0 \\
\text { Impact and contact law }
\end{array}\right.
$$

where $\operatorname{dim}\left(z_{1}\right)+\operatorname{dim}\left(z_{2}\right)=n_{1}+n_{2}=2 n, z_{1}$ and $z_{2}$ are the states (positions and velocities) of the uncontrolled and the controlled parts, respectively. The uncontrolled dynamics (29) (a) corresponds to (25) (a) or (27), while the controlled subsystem (29) (b) corresponds to (25) (b) (c) or (26) (b) (c) and (28). Another class of systems that can be recast into jugglers, are biped robots in Figure 5 (b), where the uncontrolled part corresponds to the center of mass (CoM) dynamics, the control input $\tau$ 


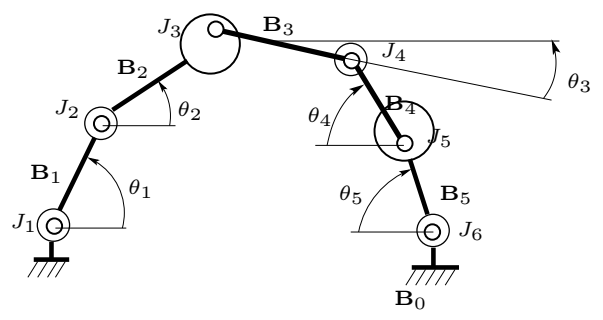

(a)

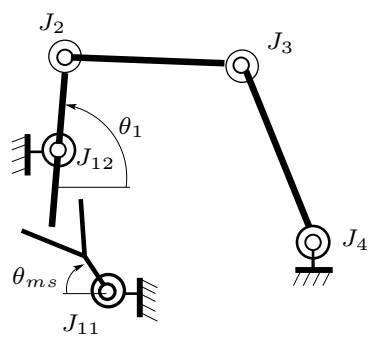

(b)

Fig. 6: (a) Six-bar mechanism with clearances at $J_{3}, J_{5}$, (b) Four-bar mechanism with gear backlash at joint $J_{1}$.

consisting of internal torques at the joints ${ }^{11}$. Running biped robots whose motion consists of airborne phases separated by impacts between the feet and the ground [29], belong to the class of juggling systems, and the control techniques which have been developped for juggling systems may also apply to running robots, as alluded to in [22], despite airborne biped robots possess other peculiarities like the whole system angular momentum (about the CoM) conservation [29], which does not make sense for systems with clearances (apart from the fact that the uncontrolled part like body $\mathbf{B}_{3}$ in (25) (a) also has its angular momentum conserved during non-contact phases). Walking biped robots have the same dynamics, however this time one has to control the uncontrolled subsystem through bounded non-negative contact forces. An abstract model of biped robot as in Figure 5 (b) where bilateral constraints have been eliminated, has eleven bodies, $n_{\text {rev }}=10$ revolute joints, $n=13, p=10$ (all ten revolute joints actuated), $m_{u}=2$ (two feet with unilateral contact $f_{1}(q) \geqslant 0$ and $\left.f_{2}(q) \geqslant 0\right), m_{u}^{\prime}=1$ (one foot in persistent contact), or $m_{u}^{\prime}=2$ (two feet in persistent contact) $\operatorname{dim}\left(z_{1}\right)=4, \operatorname{dim}\left(z_{2}\right)=22$. It is noteworthy that the canonical representation (29) is different from the canonical representation used in biped robots literature [40, Equation (5)] [75, Equation (2)] [85, Equation (2)] [6, Equation (1)], in which the uncontrolled dynamics (29) does not necessarily appear explicitly, depending on the choice of the generalized coordinates ${ }^{12}$. Coulomb friction plays a crucial role for such systems to prevent the contacting foot from slipping (hence suppressing two DoFs per sticking contact foot, creating a non-actuated joint at $A$ or $B$, hence $n=11$ during one foot in contact phases, $n=9$ during two feet in contact phases), while the sticking of contact points may not be of interest in systems with clearances, placing them in a quite different control perspective from walking biped robots. Actually the role of friction is quite different for the systems we are dealing with in this paper, where it may appear either as a disturbance (with, for instance, stick/slip behaviour) or as a stabilizing factor since it dissipates energy. Let us point out that so-called snake robots [50] possess also a juggling structure [51, Equation (2.38)], where the contact forces stem from friction. It is noteworthy in passing that if Coulomb's friction is used, the contact forces can still be expressed through complementarity conditions. Adopting the notations of section 3.3, the frictional force in the right-hand side of (23) is rewritten as $H_{\mathrm{t}, u} \lambda_{\mathrm{t}, u}=\sum_{i=1}^{m_{u}^{\prime}} H_{\mathrm{t}, u, i \bullet} \lambda_{\mathrm{t}, u, i}$, with $\lambda_{\mathrm{t}, u, i}=-\mu_{i} \lambda_{\mathrm{n}, u, i} \xi_{i}, \xi_{i} \in \operatorname{sgn}\left(v_{\mathrm{t}, u, i}\right)$, in the case of planar friction $(d=1)$. Using [18, Equation (5.122)] we have $\xi_{i} \in \operatorname{sgn}\left(v_{\mathrm{t}, u, i}\right)$ is equivalent to $0 \leqslant 1+\xi_{i} \perp \mu_{1, i} \geqslant 0,0 \leqslant 1-\xi_{i} \perp \mu_{2, i} \geqslant 0, v_{\mathrm{t}, u, i}=\mu_{1, i}-\mu_{2, i}$. This is a different kind of nonsmooth mechanical system, where the link between the two subsystems (29) (a) and (29) (b) has a different structure. Vibratory tables which are used to separate granular materials, constitute another class of jugglers with $n_{1}$ very large (number of "grains" on the table) and $n_{2}$ small (the vibratory table). Here also the link between both dynamics stems from friction (and possibly impacts). Their control is an open and challenging problem.

\footnotetext{
11 From the Fundamental Principle of Dynamics, the CoM's dynamics of a material system depends only on the external forces acting on the system.

12 As written in [40] about biped robots: the choice of the generalized coordinates $q$ is crucial for control purpose, and certainly much less obvious than it is for serial manipulators. This could be extended to systems with joint clearance.
} 
The controllability properties and the control of juggling systems have been investigated in $[22,57,53,91$, 20,55], where dead-beat control is a central tool. Most of these results apply to the all-linear case (linear dynamics and linear constraints). We may nevertheless try to apply and/or extend the ideas outlined in $[22, \S$ III.C $]$ for the multi-input/multi-output case, to (25). The basic idea is to give desired values to the densities at impact times (with respect to the Dirac measure) of both $\lambda_{\mathrm{n}, u, 12}$ and $\lambda_{\mathrm{n}, u, 23}$, which are the non-negative impulses $p_{\mathrm{n}, u, 12}\left(t_{k}\right)$ and $p_{\mathrm{n}, u, 23}\left(t_{k}\right)$, in order to control the dynamics (25) (a), using suitable $\tau_{1}$ and $\tau_{3}$ between impacts. Due to the strong nonlinearity of the gap functions, it is however not an easy task to calculate suitable impact points between the bearing and the journal at both $J_{3}$ and $J_{4}$, in order to impose a desired trajectory to $\mathbf{B}_{3}$. To simplify this design, let us examine first the input/output (I/O) linearization of the controlled system (25) (b) (c) with output $y=\left(y_{1}, y_{2}\right)^{T}, y_{1}=f_{12}\left(q_{1}, q_{2}\right)$ and $y_{2}=f_{23}\left(q_{2}, q_{3}\right)$. Since $p=4$ and $m_{u}=2$, one has to consider an input of dimension two to perform the $\mathrm{I} / \mathrm{O}$ linearization. Calculations yield (arguments are dropped):

$$
\begin{aligned}
\ddot{y}= & \underbrace{\left(\begin{array}{c}
\mathcal{M}_{12}^{T} M_{1}^{-1} \\
\mathcal{M}_{32}^{T} M_{3}^{-1}
\end{array}\right)}_{\triangleq_{\mathcal{D}(q) \in \mathbb{R}^{2 \times 4}}} \underbrace{\left(\begin{array}{c}
\tau_{1} \\
\tau_{3}
\end{array}\right)}_{\triangleq_{\tau}}+\underbrace{\left(\begin{array}{c}
\mathcal{M}_{21}^{T} M_{2}^{-1} \mathcal{M}_{21}+\mathcal{M}_{12}^{T} M_{1}^{-1} \mathcal{M}_{12} \\
\mathcal{M}_{23}^{T} M_{2}^{-1} \mathcal{M}_{21} \mathcal{M}_{21}^{T} M_{2}^{-1} \mathcal{M}_{23} \\
\mathcal{M}_{23}^{T} M_{2}^{-1} \mathcal{M}_{23}+\mathcal{M}_{32}^{T} M_{3}^{-1} \mathcal{M}_{32}
\end{array}\right)}_{\triangleq_{\mathcal{M}(q)=\mathcal{M}(q))^{T} \succeq 0}} \underbrace{\left(\begin{array}{c}
\lambda_{\mathrm{n}, u, 12} \\
\lambda_{\mathrm{n}, u, 23}
\end{array}\right)}_{\triangleq_{\lambda_{\mathrm{n}, u}}} \\
& \underbrace{-\left(\begin{array}{c}
\mathcal{M}_{12}^{T} M_{1}^{-1} F_{1} \\
\mathcal{M}_{23}^{T} M_{2}^{-1} G_{2}+\mathcal{M}_{32}^{T} M_{3}^{-1} F_{3}
\end{array}\right)+\left(\begin{array}{c}
\frac{d}{d t}\left(\mathcal{M}_{21}^{T}\right) \dot{q}_{2}+\frac{d}{d t}\left(\mathcal{M}_{12}^{T}\right) \dot{q}_{1} \\
\frac{d}{d t}\left(\mathcal{M}_{23}^{T}\right) \dot{q}_{2}+\frac{d}{d t}\left(\mathcal{M}_{32}^{T}\right) \dot{q}_{3}
\end{array}\right)}_{\triangleq_{\mathcal{F}(q, \dot{q})}} .
\end{aligned}
$$

Assume that the decoupling matrix satisfies $\operatorname{rank}(\mathcal{D}(q))=2$ for all $q \in \Phi$, then its Moore-Penrose inverse $\mathcal{D}(q)^{\dagger}=\mathcal{D}(q)^{T}\left(\mathcal{D}(q) \mathcal{D}(q)^{T}\right)^{-1} \in \mathbb{R}^{4 \times 2}$. Due to the rank of $\mathcal{D}(q), \operatorname{dim}[\operatorname{ker}(\mathcal{D}(q))]=2$ [10, Corollary 2.5.5], and $\left(I_{4}-\mathcal{D}(q)^{\dagger} \mathcal{D}(q)\right)$ is the projector onto $\operatorname{ker}(\mathcal{D}(q))$ [10, Proposition 6.1 .6 xii)]. Let $\tilde{\tau} \in \mathbb{R}^{4}$, $\tau_{\text {ker }}=\left(I_{4}-\mathcal{D}(q)^{\dagger} \mathcal{D}(q)\right) \tilde{\tau}$, and $\bar{\tau} \in \mathbb{R}^{2}$. Setting

$$
\tau=\mathcal{D}(q)^{\dagger}[\bar{\tau}-\mathcal{F}(q, \dot{q})]+\tau_{\text {ker }}
$$

yields the I/O operator $\ddot{y}=\bar{\tau}+\mathcal{M}(q) \lambda_{\mathrm{n}, u}$. When $y>0 \Rightarrow \lambda_{\mathrm{n}, u}=0$, this I/O operator is linear and the controller $\bar{\tau}$ can be used to drive the new state $\left(y^{T}, \dot{y}^{T}\right)^{T} \in \mathbb{R}^{4}$ between two arbitrary boundary values within an arbitrary time interval. Therefore the $\mathrm{I} / \mathrm{O}$ linearization is made between $\bar{\tau}$ and $y$, with decoupling matrix $\mathcal{D}(q) \mathcal{D}(q)^{\dagger} \in \mathbb{R}^{2 \times 2}$ which is the projector onto $\operatorname{im}(\mathcal{D}(q))$ [10, Proposition $\left.6.1 .6 \mathrm{x}\right)$ ], and from [10, Proposition 6.1.6 xv)] $\operatorname{rank}\left(\mathcal{D}(q) \mathcal{D}(q)^{\dagger}\right)=2$ for all $q$ : the system has a uniform relative degree $r=(2,2)^{T}$. Until now we have replaced the state $\left(q_{2}^{T}, \dot{q}_{2}^{T}, q_{1}^{T}, \dot{q}_{1}^{T}, q_{3}^{T}, \dot{q}_{3}^{T}\right)^{T} \in \mathbb{R}^{14}$ of (25) (a) (b) (c) by $\left(q_{2}^{T}, \dot{q}_{2}^{T}, y^{T}, \dot{y}^{T}\right)^{T} \in \mathbb{R}^{10}$. The remaining part of the transformed state vector is the so-called zero dynamics of the controlled subsystem with input $\bar{\tau}$ and output $y$. The zero dynamics has a state $\xi \in \mathbb{R}^{4}$. There are various forms for the zero dynamics, and various existence conditions [23]. Due to lack of space we do not provide a detailed analysis on the zero dynamics (see [23, Proposition 3.2b, Corollaries 5.6 and $5.7])$, and we conjecture that it is given as $\dot{\xi}=\Xi_{z}(\xi, y)+\Xi_{\tau}(z) \tilde{\tau}+\Xi_{\lambda}(z) \lambda_{\mathrm{n}}$ for some matrix functions $\Xi_{z}(\cdot), \Xi_{\tau}(\cdot), \Xi_{\lambda}(\cdot)^{13}$. Since two inputs are sufficient to realize the I/O linearization, $\tau_{\text {ker }}$ is the part of the input $\tau$ which may be used to control the remaining part of the dynamics, i.e. the zero dynamics. It is important to note that $\tau_{\text {ker }} \in \mathbb{R}^{4}$, but it belongs to a two-dimensional subspace, hence it provides only two independent inputs, whatever the value of $\tilde{\tau}$. To summarize, under the various assumptions we made, letting $z_{3}=y$ and $z_{4}=\dot{y}$, the system (25) (a) (b) (c) with output $y$ is assumed to be (locally or

\footnotetext{
13 It is however worth pointing out that despite existence conditions can be analysed, the explicit calculation of the zero dynamics may be hard.
} 
globally) transformed into the canonical form:

$$
\left\{\begin{array}{l}
\left(\text { a) } M_{2} \ddot{q}_{2}+G_{2}\left(q_{2}\right)=\left(\mathcal{M}_{21}\left(q_{2}, q_{1}\right), \mathcal{M}_{23}\left(q_{2}, q_{3}\right)\right) \lambda_{\mathrm{n}, u}\right. \\
\text { (b) } \dot{z}_{3}=z_{4} \\
\dot{z}_{4}=\bar{\tau}+\mathcal{M}(q) \lambda_{\mathrm{n}, u} \\
\text { (c) } \dot{\xi}=\Xi_{z}(\xi, y)+\Xi_{\tau}(z) \tilde{\tau}+\Xi_{\lambda}(z) \lambda_{\mathrm{n}, u} \\
(d) 0 \leqslant \lambda_{\mathrm{n}, u} \perp y=z_{3} \geqslant 0 \\
(e) 0 \leqslant \dot{y}_{i}\left(t_{k}^{+}\right)+e_{\mathrm{n}} \dot{y}_{i}\left(t_{k}^{-}\right) \perp p_{\mathrm{n}, u, i}(t) \geqslant 0, \text { when } y_{i}\left(t_{k}\right)=0 \text { and } \dot{y}_{i}\left(t_{k}^{-}\right) \leqslant 0, i=1,2,
\end{array}\right.
$$

where the impact law stems from (4). As alluded to in $[22, \S I I I . B]$, the zero dynamics of the complete system (32) with $\lambda_{\mathrm{n}, u}=0$, is represented by the $\xi$ and the $q_{2}$ dynamics. When only vibro-impact dynamics is considered (no persistent contact phases), it renders (32) close to the dynamics in [5, Equation (49)] introduced for biped robots control.

Remark 3 (Persistent contact with positive control) The canonical form in (32), which is no longer a Lagrangian system, when replaced in the general context of (29), corresponds to a partial I/O linearization between $y=f\left(z_{1}, z_{2}\right)$ and one part of $\tau$, where in general $\operatorname{dim}(\tau)>\operatorname{dim}(y)$. The controlled subsystem's zero dynamics represents the system's dynamics on the submanifold $\mathcal{Z}_{0}=\left\{z \in \mathbb{R}^{14} \mid y(t)=z_{3}(t)=\right.$ 0 for all $t \geqslant 0\}$. In $\mathcal{Z}_{0}$ one has $\bar{\tau}=-\mathcal{M}(q) \lambda_{\mathrm{n}, u}$ and $\dot{\xi}=\Xi_{z}(\xi, 0)+\Xi_{\tau}(z) \tilde{\tau}+\Xi_{\lambda}(z) \lambda_{\mathrm{n}, u}$ where $\lambda_{\mathrm{n}, u}$ is the solution of the contact LCP (see (8)). Thus the persistent contact control analysed in section 3 , is control in $\mathcal{Z}_{0}$. In persistent contact with both constraints we obtain $\lambda_{\mathrm{n}, u}=-\mathcal{M}(q)^{-1} \bar{\tau}>0$, which can be guaranteed if and only if there exists $\bar{\tau}$ such that $\mathcal{M}(q)^{-1} \bar{\tau}=-\beta(t), \beta(t)>0$ (this replaces the condition $\hat{E}(q) \tau_{\beta}-\hat{w}(q, \dot{q})<0$ in $\left.(13)\right)$, or equivalently if there exists $\beta(t)>0$ such that $\bar{\tau}=-\mathcal{M}(q) \beta(t)<0$. Setting $z_{3}(0)=z_{4}\left(0^{+}\right)=0$ we obtain from $(32)$ the control problem with positive controls:

$$
\left\{\begin{array}{l}
\left(\text { a) } M_{2} \ddot{q}_{2}+G_{2}\left(q_{2}\right)=\left(\mathcal{M}_{21}\left(q_{2}, q_{1}\right), \mathcal{M}_{23}\left(q_{2}, q_{3}\right)\right) \beta(t)\right. \\
(b) \dot{z}_{3}=z_{4}=0 \\
\dot{z}_{4}=\bar{\tau}+\mathcal{M}(q) \lambda_{\mathrm{n}, u}=0 \\
(c) \dot{\xi}=\Xi_{z}(\xi, y)+\Xi_{\tau}(z) \tilde{\tau}+\Xi_{\lambda}(z) \beta(t) \\
\beta(t)>0, \quad \bar{\tau}=-\mathcal{M}(q) \beta(t)
\end{array}\right.
$$

which makes an alternative to (21) in order to analyse the contact controllability.

Remark 4 Let us consider instead the four-bar system in Figure 4 (b) with clearances at joints $J_{2}$ and $J_{3}$, and actuators at $J_{1}$ and $J_{4}$. Then $m_{u}=2$ and $p=2$, and the dynamics can be written as (25) with lower dimensions of $q_{1}=\theta_{1} \in \mathbb{R}$ and $q_{3}=\theta_{3} \in \mathbb{R}$. So the system a priori fits within the framework for $\mathrm{I} / \mathrm{O}$ linearization (provided its decoupling matrix has full-rank), without zero dynamics (32) (c), which makes the control design easier. Following [22, Lemma 4], the presented methodology better suits to systems with $\operatorname{dim}\left(z_{2}\right)=r$. As a further example of a mechanism with clearances that naturally fits within the class of jugglers, we may consider a planar three-DoF parallel manipulator as in $[65, \S 6]$ which has seven bodies and nine revolute joints. If clearances are present at joints $C_{1}, C_{2}$ and $C_{3}$, it has nine DoFs. Its uncontrolled dynamics is body seven, and the three (underactuated) controlled parts are bodies 1 and 2 , 3 and 4, 5 and 6 (see [65, Figure 1] for notations). This is a direct extension of the six-bar mechanism in Figure $5(\mathrm{a})$.

Let us now outline the extension of the three-step recursive method proposed in $[22 \text {, } \S \text { III.B }]^{14}$, to (32), keeping in mind that it could be generalized to (29). Next we consider a particular motion of (32) (a) that consists of successive single impacts with $\Sigma_{i} \triangleq\left\{q \in \mathcal{C} \mid y_{i}=0\right\}, i=1,2$, with $y_{1}\left(t_{2 k}\right)=0$, $y_{2}\left(t_{2 k}\right)>0, y_{2}\left(t_{2 k+1}\right)=0, y_{1}\left(t_{2 k+1}\right)>0, k \geqslant 0$. That is, we consider the mapping $\Sigma_{1}^{+} \rightarrow \Sigma_{2}^{+} \rightarrow \Sigma_{1}^{+}$, with $\Sigma_{1}^{+} \triangleq\left\{z \in \mathbb{R}^{14} \mid y_{1}=0, \dot{y}_{1}>0, y_{2}>0\right\}, \Sigma_{2}^{+} \triangleq\left\{z \in \mathbb{R}^{14} \mid y_{2}=0, \dot{y}_{2}>0, y_{1}>0\right\}$, with $y_{1}=z_{3,1}$,

\footnotetext{
14 This originates from [22,90], and it has also been used in [57]. It may be seen as an extension of the popular backstepping control method, see in particular the developments in $[53, \S 4]$ for an illustration.
} 
$y_{2}=z_{3,2}$. Clearly other options exist, and this renders the overall control problem rather tough. It is noteworthy that if there is no zero dynamics, and $G\left(q_{2}\right)=0$, the controllability issue is still highly non trivial [20]. We assume that $\mathcal{M}(q) \succ 0$ for all $q \in \mathcal{C}$ in (30). On intervals $\left(t_{k}, t_{k+1}\right)$, we denote $q_{2}\left(t_{k+1}\right)=\phi_{2}^{p}\left(q_{2}\left(t_{k}\right), \dot{q}_{2}\left(t_{k}^{+}\right), \Delta_{k}\right)$, with $\Delta_{k}=t_{k+1}-t_{k}$, and $\dot{q}_{2}\left(t_{k+1}^{-}\right)=\phi_{2}^{v}\left(q_{2}\left(t_{k}\right), \dot{q}_{2}\left(t_{k}^{+}\right), \Delta_{k}\right)$. From the impact dynamics and the restitution law we have when an impact occurs with $\Sigma_{1}$ :

$$
\begin{aligned}
\dot{q}_{2}\left(t_{2 k}^{+}\right) & =\phi_{\Sigma_{1}}^{i m p}\left(q\left(t_{2 k}\right), \dot{q}_{2}\left(t_{2 k}^{-}\right), e_{\mathrm{n}}, \dot{y}_{1}\left(t_{2 k}^{-}\right)\right) \\
& =\dot{q}_{2}\left(t_{2 k}^{-}\right)+M_{2}^{-1}\left[\mathcal{M}_{21}\left(q_{2}\left(t_{2 k}\right), q_{1}\left(t_{2 k}\right)\right), \mathcal{M}_{23}\left(q_{2}\left(t_{2 k}\right), q_{3}\left(t_{2 k}\right)\right)\right] \mathcal{M}\left(q\left(t_{2 k}\right)\right)^{-1}\left(\begin{array}{c}
-\left(1+e_{\mathrm{n}}\right) \dot{y}_{1}\left(t_{2 k}^{-}\right) \\
0
\end{array}\right)
\end{aligned}
$$

and we obtain similarly $\phi_{\Sigma_{2}}^{i m p}\left(q\left(t_{2 k+1}\right), \dot{q}_{2}\left(t_{2 k+1}^{-}\right), e_{\mathrm{n}}, \dot{y}_{2}\left(t_{2 k+1}^{-}\right)\right)$for an impact with $\Sigma_{2}$. Using the state transformation we also have $q_{1}=\mathcal{X}_{1}\left(q_{2}, z_{3}, \xi\right)$ and $q_{3}=\mathcal{X}_{3}\left(q_{2}, z_{3}, \xi\right)$, while $q_{2}=z_{1}$, for some functions $\mathcal{X}_{1}(\cdot)$ and $\mathcal{X}_{3}(\cdot)$. A basic requirement for what follows, is that all foregoing mappings are calculable.

1. Calculate the mappings $\Sigma_{1}^{+} \rightarrow \Sigma_{2}^{+} \rightarrow \Sigma_{1}^{+}$. To this aim we start from:

$$
\begin{aligned}
q_{2}\left(t_{2 k+2}\right)= & \phi_{2}^{p}\left(q_{2}\left(t_{2 k+1}\right), \dot{q}_{2}\left(t_{2 k+1}^{+}\right), \Delta_{2 k+1}\right) \\
= & \phi_{2}^{p}\left(\phi_{2}^{p}\left[q_{2}\left(t_{2 k}\right), \dot{q}_{2}\left(t_{2 k}^{+}\right), \Delta_{2 k}\right], \phi_{\Sigma_{2}}^{i m p}\left[q\left(t_{2 k+1}\right), \dot{q}_{2}\left(t_{2 k+1}^{-}\right), e_{\mathrm{n}}, \dot{y}_{2}\left(t_{2 k+1}^{-}\right)\right], \Delta_{2 k+1}\right) \\
\dot{q}_{2}\left(t_{2 k+2}^{+}\right)= & \phi_{\Sigma_{1}}^{i m p}\left(q\left(t_{2 k+2}\right), \dot{q}_{2}\left(t_{2 k+2}^{-}\right), e_{\mathrm{n}}, \dot{y}_{1}\left(t_{2 k+2}^{-}\right)\right) \\
= & \phi_{\Sigma_{1} p}^{i m p}\left(q\left(t_{2 k+2}\right), \phi_{2}^{v}\left(q_{2}\left(t_{2 k+1}\right), \dot{q}_{2}\left(t_{2 k+1}^{+}\right), \Delta_{2 k+1}\right), e_{\mathrm{n}}, \dot{y}_{1}\left(t_{2 k+2}^{-}\right)\right) \\
= & \phi_{\Sigma_{1}}^{i m p}\left(q\left(t_{2 k+2}\right), \phi_{2}^{v}\left(\phi_{2}^{p}\left[q_{2}\left(t_{2 k}\right), \dot{q}_{2}\left(t_{2 k}^{+}\right), \Delta_{2 k}\right], \phi_{\Sigma_{2}}^{i m p}\left[q\left(t_{2 k+1}\right), \dot{q}_{2}\left(t_{2 k+1}^{-}\right), e_{\mathrm{n}}, \dot{y}_{2}\left(t_{2 k+1}^{-}\right)\right], \Delta_{2 k+1}\right),\right. \\
& \left.\quad e_{\mathrm{n}}, \dot{y}_{1}\left(t_{2 k+2}^{-}\right)\right)
\end{aligned}
$$

with

$$
\begin{aligned}
& \phi_{\Sigma_{2}}^{i m p}\left(q\left(t_{2 k+1}\right), \dot{q}_{2}\left(t_{2 k+1}^{-}\right), e_{\mathrm{n}}, \dot{y}_{2}\left(t_{2 k+1}^{-}\right)\right)=\phi_{\Sigma_{2}}^{i m p}\left(q\left(t_{2 k+1}\right), \phi_{2}^{v}\left[q_{2}\left(t_{2 k}\right), \dot{q}_{2}\left(t_{2 k}^{+}\right), \Delta_{2 k}\right], e_{\mathrm{n}}, \dot{y}_{2}\left(t_{2 k+1}^{-}\right)\right) \\
& q\left(t_{2 k+1}\right)=\left(\begin{array}{c}
\mathcal{X}_{1}\left(\phi_{2}^{p}\left(q_{2}\left(t_{2 k}\right), \dot{q}_{2}\left(t_{2 k}^{+}\right), \Delta_{2 k}\right), y_{1}\left(t_{2 k+1}\right), 0, \xi\left(t_{2 k+1}\right)\right) \\
\phi_{2}^{p}\left(q_{2}\left(t_{2 k}\right), \dot{q}_{2}\left(t_{2 k}^{+}\right), \Delta_{2 k}\right) \\
\mathcal{X}_{3}\left(\phi_{2}^{p}\left(q_{2}\left(t_{2 k}\right), \dot{q}_{2}\left(t_{2 k}^{+}\right), \Delta_{2 k}\right), y_{1}\left(t_{2 k+1}\right), 0, \xi\left(t_{2 k+1}\right)\right)
\end{array}\right) \\
& \triangleq \phi^{p}\left(q_{2}\left(t_{2 k}\right), \dot{q}_{2}\left(t_{2 k}^{+}\right), \Delta_{2 k}, y_{1}\left(t_{2 k+1}\right), \xi\left(t_{2 k+1}\right)\right) \\
& \xi\left(t_{2 k+1}\right)=\phi^{\xi}\left(\xi\left(t_{2 k}\right), \bar{\tau}_{[2 k, 2 k+1]}, \tilde{\tau}_{[2 k, 2 k+1]}\right)
\end{aligned}
$$

where $\bar{\tau}_{[k, k+1]}$ and $\tilde{\tau}_{[k, k+1]}$ generically denote the action of the controllers over the interval $\left(t_{k}, t_{k+1}\right)$, and we note that $\bar{\tau}$ influences $\xi$ through its dependence on $y$. We recall that $y_{1}\left(t_{2 k+2}\right)=y_{2}\left(t_{2 k+1}\right)(=$ $\left.y_{1}\left(t_{2 k}\right)\right)=0, y_{1}\left(t_{2 k+1}\right)>0, y_{2}\left(t_{2 k}\right)>0, y_{1}\left(t_{2 k+2}\right)>0, \dot{y}_{2}\left(t_{2 k+1}^{-}\right)<0, \dot{y}_{1}\left(t_{2 k}^{-}\right)<0, \dot{y}_{1}\left(t_{2 k+2}^{-}\right)<0$. Similar manipulations as in (36) yield:

$$
\left\{\begin{array}{l}
q\left(t_{2 k+2}\right)=\phi^{p}\left(q_{2}\left(t_{2 k+1}\right), \dot{q}_{2}\left(t_{2 k+1}^{+}\right), \Delta_{2 k+1}, y_{1}\left(t_{2 k+2}\right), \xi\left(t_{2 k+2}\right)\right) \\
\xi\left(t_{2 k+2}\right)=\phi^{\xi}\left(\xi\left(t_{2 k+1}\right), \bar{\tau}_{[2 k+1,2 k+2]}, \tilde{\tau}_{[2 k+1,2 k+2]}\right)
\end{array}\right.
$$

From (32) (b) we deduce the existence of the mappings:

$$
\left\{\begin{array}{l}
\dot{y}_{2}\left(t_{2 k+1}^{-}\right)=\phi_{2}^{\dot{y}}\left(t_{2 k+1}^{-} ; y_{2}\left(t_{2 k}\right), \dot{y}_{2}\left(t_{2 k}^{+}\right), \bar{\tau}_{[2 k, 2 k+1]}\right) \\
\dot{y}_{1}\left(t_{2 k+2}^{-}\right)=\phi_{1}^{\dot{y}}\left(t_{2 k+1}^{-} ; y_{1}\left(t_{2 k+1}\right), \dot{y}_{1}\left(t_{2 k+1}^{+}\right), \bar{\tau}_{[2 k+1,2 k+2]}\right) \\
y_{1}\left(t_{2 k+2}\right)=\phi_{1}^{y}\left(t_{2 k+1} ; y_{1}\left(t_{2 k+1}\right), \dot{y}_{1}\left(t_{2 k+1}^{+}\right), \bar{\tau}_{[2 k+1,2 k+2]}\right) \\
y_{2}\left(t_{2 k+1}\right)=\phi_{2}^{y}\left(t_{2 k+1} ; y_{2}\left(t_{2 k}\right), \dot{y}_{2}\left(t_{2 k}^{+}\right), \bar{\tau}_{[2 k, 2 k+1]}\right) \\
y_{1}\left(t_{2 k+1}\right)=\phi_{1}^{y}\left(t_{2 k+1} ; y_{1}\left(t_{2 k}\right), \dot{y}_{1}\left(t_{2 k}^{+}\right), \bar{\tau}_{[2 k, 2 k+1]}\right) \\
y_{2}\left(t_{2 k+2}\right)=\phi_{2}^{y}\left(t_{2 k+2} ; y_{2}\left(t_{2 k+1}\right), \dot{y}_{2}\left(t_{2 k+1}^{+}\right), \bar{\tau}_{[2 k+1,2 k+2]}\right)
\end{array}\right.
$$

Now using (35), (36), (37) we infer the existence of mappings $\Sigma_{1}^{+} \rightarrow \Sigma_{2}^{+} \rightarrow \Sigma_{1}^{+}$:

$$
\left\{\begin{array}{l}
q_{2}\left(t_{2 k+2}\right)=\Phi_{2}^{p}\left(\dot{y}_{2}\left(t_{2 k+1}^{-}\right), \dot{y}_{1}\left(t_{2 k+2}^{-}\right) ; \Delta_{2 k}, \Delta_{2 k+1}, \dot{q}_{2}\left(t_{2 k}^{+}\right), q_{2}\left(t_{2 k}\right), \xi\left(t_{2 k+1}\right)\right) \\
\dot{q}_{2}\left(t_{2 k+2}^{+}\right)=\Phi_{2}^{v}\left(\dot{y}_{2}\left(t_{2 k+1}^{-}\right), \dot{y}_{1}\left(t_{2 k+2}^{-}\right) ; \Delta_{2 k}, \Delta_{2 k+1}, \dot{q}_{2}\left(t_{2 k}^{+}\right), q_{2}\left(t_{2 k}\right), \xi\left(t_{2 k+1}\right), \xi\left(t_{2 k+2}\right)\right)
\end{array}\right.
$$


2. Given $q_{2}\left(t_{2 k}\right)$ and $\dot{q}_{2}\left(t_{2 k}^{+}\right)$, fix desired values $q_{2, d}\left(t_{2 k+2}\right)$ and $\dot{q}_{2, d}\left(t_{2 k+2}^{+}\right)$, and use (39) to calculate "fictitious" inputs $\dot{y}_{2}^{\star}\left(t_{2 k+1}^{-}\right), \dot{y}_{1}^{\star}\left(t_{2 k+2}^{-}\right), \Delta_{2 k}^{\star}, \Delta_{2 k+1}^{\star}, \xi^{\star}\left(t_{2 k+1}\right), \xi^{\star}\left(t_{2 k+2}\right)$.

3. Use (36) (37) and (38) to calculate $\bar{\tau}$ and $\tilde{\tau}$ on $\left[t_{2 k}, t_{2 k+1}\right]$ and $\left[t_{2 k+1}, t_{2 k+2}\right]$ such that $\dot{y}_{2}\left(t_{2 k+1}^{-}\right)=$ $\dot{y}_{2}^{\star}\left(t_{2 k+1}^{-}\right), \dot{y}_{1}\left(t_{2 k+2}^{-}\right)=\dot{y}_{1}^{\star}\left(t_{2 k+2}^{-}\right), y_{1}\left(t_{2 k+2}\right)=0, y_{2}\left(t_{2 k+1}\right)=0, \Delta_{2 k}=\Delta_{2 k}^{\star}, \Delta_{2 k+1}=\Delta_{2 k+1}^{\star}$, $\xi\left(t_{2 k+1}\right)=\xi^{\star}\left(t_{2 k+1}\right), \xi\left(t_{2 k+2}\right)=\xi^{\star}\left(t_{2 k+2}\right)$.

4. Check the viability, i.e. $y_{1}(t)>0$ for all $t \in\left(t_{2 k}, t_{2 k+2}\right)$ and $y_{2}(t)>0$ for all $t \in\left[t_{2 k}, t_{2 k+1}\right) \cup$ $\left(t_{2 k+1}, t_{2 k+2}\right]$.

At step 2, the intermediate values $q_{2}\left(t_{2 k+1}\right)$ and $\dot{q}_{2}\left(t_{2 k+1}^{-}\right)$are not specified, but they could be. As alluded to above, other choices of impact sequences can be made. However if the two constraints are programmed to be hit simultaneously (a multiple, two-impact occurs), then issues related to discontinuity with respect to initial data should be examined as they may influence robustness and stability. Specific controllers are proposed for step 3 in $[22,53,55,57]$, including also step 4 . Clearly various strategies may be adopted (impulsive, piecewise-constant, optimal control). It is noteworthy that step 2 may fail: more intermediate collisions may be needed to reach the desired states. Moreover the above algorithm may be made more stringent by imposing desired values to $\dot{y}_{1}\left(t_{2 k+2}^{+}\right)$and $\dot{y}_{2}\left(t_{2 k+2}^{+}\right)$(as done in [53] for the system in Figure 2 (b)). Actually, the real challenge is to derive criteria which guarantee that desired, arbitrary states, can be attained after a given number of collisions (and possibly of persistent contact phases, see remark $4)$. This is the goal of [20] for simple jugglers where the mappings $\phi_{2}^{p}(\cdot), \phi_{2}^{v}(\cdot)$ are easily calculable, see also [22, Lemma 3]. More generally this requires further characterization of the different mappings and flows involved in the recursive method. Efficient numerical algorithms can certainly be set to cope with this issue, recursively computing attainable subsets. Finally we point out that the results in [5], using control-Lyapunov functions and hybrid zero dynamics for systems quite close to (29) if in a vibro-impact regime, could be tried as well in our framework (though the required invariance conditions in [5, §V.A] may be stringent in our case).

Instead of an I/O linearization one may apply a state feedback linearization with $\tau_{i}=M_{i}\left(q_{i}\right)+F_{i}\left(q_{i}, \dot{q}_{i}\right)+$ $\bar{\tau}_{i}, i=1,3$, so that we obtain instead of $(32)$ :

$$
\left\{\begin{array}{l}
\text { (a) } M_{2} \ddot{q}_{2}+G_{2}\left(q_{2}\right)=\left(\mathcal{M}_{21}\left(q_{2}, q_{1}\right), \mathcal{M}_{23}\left(q_{2}, q_{3}\right)\right) \lambda_{\mathrm{n}, u} \\
\text { (b) } \ddot{q}_{1}=\bar{\tau}_{1}+\mathcal{M}_{12}\left(q_{1}, q_{2}\right) \lambda_{\mathrm{n}, u, 12} \\
\text { (c) } \ddot{q}_{3}=\bar{\tau}_{3}+\mathcal{M}_{32}\left(q_{2}, q_{3}\right) \lambda_{\mathrm{n}, u, 32} \\
\text { (d) Complementarity conditions in (25) (d) } \\
\text { (e) Impact law in (25) (e) }
\end{array}\right.
$$

Similar steps as above can be followed, though some mappings may be less easy to calculate due to nonlinear gap functions. However the zero dynamics (when it exists) does not have to be computed. The choice between (32) and (40) thus depends on the system's input/ouput properties. When the gap functions are linear and/or there is no zero dynamics, the design process simplifies drastically, and the controller proposed in $[55,57]$ could be applied to drive the whole state to the origin in finite time (hence solving with a totally different approach, the problem of null-controllability of section 3). The developments of Remark 3 can be applied to (40), but the analysis on the constraint surface is less easy if the constraints are nonlinear.

Remark 5 Other results on controllability and control of jugglers have been obtained elsewhere, see e.g. [52]. However they rely on the strong assumption that the controlled part ((25 (b) (c) or (26) (b) (c) and (28) in our case) is not affected by impacts. Though this assumption may be reasonable in some juggling systems, it may not hold at all in kinematic chains with joint clearance. It does not hold neither in running and hopping bipeds, because feet/ground impacts strongly influence the whole system's dynamics, not only the CoM's dynamics. Some other robotic tasks named batting tasks and nonprehensile manipulation can be recast into the control of juggling systems, where the uncontrolled dynamics trajectories may obey relatively complex aerodynamical laws [78] and needs numerical integration for feedback purpose. Another important remark is that we did not investigate the influence on the control properties, of the particular form of the contraints. It is not equivalent to manipulate an "object" like body $\mathbf{B}_{3}$ in (25) (a) with fingers, or through revolute joints with clearance. 
4.3 Switching controller: persistent contact-impacts

Remark 6 (Switching controller) Consider Example 4, with $\tau_{2}=0$ (only body 1 is actuated). Contact may be maintained with the boundary $f_{1}(q)=0$ only if $\tau_{\beta_{1}}>0$. If $\tau_{\beta_{1}}<0$ then detachment from $f_{1}(q)=0$ occurs, and the system passes through an unconstrained phase of motion. This is necessary if one desires trajectories with changing direction. The control strategy may be to switch between several controllers which guarantee some sequence of contact modes: i) persistent contact with boundary $f_{1}(q)=0$ while $f_{2}(q)>0$, ii) unconstrained phase with $f_{1}(q)>0$ and $f_{2}(q)>0$, iii) impact with boundary $f_{2}(q)=0$ and finite-time stabilisation on it, iv) persistent contact with boundary $f_{2}(q)=0$ while $f_{1}(q)>0$, v) repeat ii), vi) impact with boundary $f_{1}(q)=0$ and finite-time stabilisation on it, vii) repeat from i). This is analysed in $[53, \S 5]$. The finite-time stabilization with finite-number of impacts has been studied in [55, 57], who in passing prove controllability through impacts. It would be interesting to combine the results for persistent contact (see Proposition 2) and those in $[55,57]$ to enlarge the scope of these studies. The set-valued twisting sliding-mode controller proposed in [64] is a relatively simple input that can drive both $y$ and $\dot{y}$ to zero in finite time, after an accumulation of impacts, and could be used at steps iii) and vi) if viability holds.

\subsection{Complete controllability of a compliant contact model}

Let us consider the one DoF clearance model as in Figure 2. Each contact is modeled with a linear spring-dashpot system, which lends itself to a complementarity representation [18, Chapter 2] [14]. Our objective is thus first to express the system as a Linear Complementarity System (LCS), then to use the controllability criterion proposed in [24].

\section{Stabilization}

The stabilization problem basically relies on Lejeune Dirichlet (or Lagrange-Dirichlet) theorem, extended to unilaterally constrained mechanical systems in $[18, \S 7.5][15][47$, Chapters 6, 7] [87]. The notion of control collocation plays a central role as shown in $[53, \S 3]$ for the PD control of the one DoF prismatic joint in Figure 2. Let us illustrate the use of the Lagrange-Dirichlet Theorem and of the KrasovskiiLaSalle invariance principle with the six-bar system in Figure 7 (a). Consider for instance that clearance is present at the joints $J_{4}, J_{5}$ and $J_{6}$, hence $S_{1}=\left(\mathbf{B}_{1}, \mathbf{B}_{2}, \mathbf{B}_{3}\right), S_{2}=\left(\mathbf{B}_{4}\right), S_{3}=\left(\mathbf{B}_{5}\right)$. Let us analyse

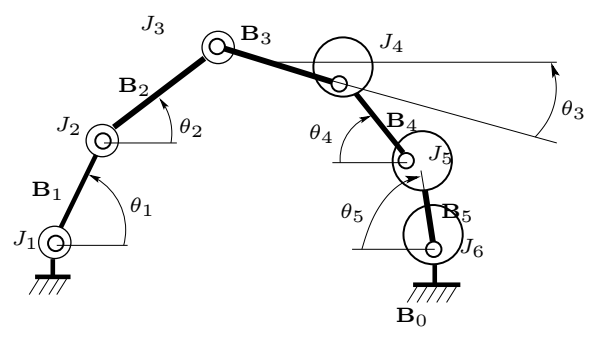

(a)

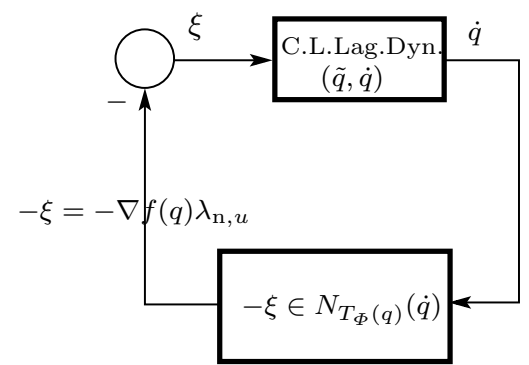

(b)

Fig. 7: (a) Clearances at $J_{4}, J_{5}$ and $J_{6}$. (b) Interconnected closed-loop in (45).

the stabilisation with a collocated controller. We suppose that joints $J_{1}, J_{2}$ and $J_{3}$ are actuated, so that 
$S_{1}$ is a fully actuated manipulator with three DoFs. The control goal is to stabilize $q_{1}=\left(\theta_{1}, \theta_{2}, \theta_{3}\right)^{T}$ at some constant values $\theta_{1, d}, \theta_{2, d}$ and $\theta_{3, d}$. The unconstrained system has the nine-DoF dynamics:

$$
\begin{cases}(a) & M_{1}\left(q_{1}\right) \ddot{q}_{1}+C_{1}\left(q_{1}, \dot{q}_{1}\right) \dot{q}_{1}+G_{1}\left(q_{1}\right)=\tau_{1}+\mathcal{M}_{14}\left(q_{1}, q_{4}\right) \lambda_{\mathrm{n}, u, 14} \\ \text { (b) } & M_{4} \ddot{q}_{4}+G_{4}\left(q_{4}\right)=\mathcal{M}_{45}\left(q_{4}, q_{5}\right) \lambda_{\mathrm{n}, u, 45}+\mathcal{M}_{41}\left(q_{1}, q_{4}\right) \lambda_{\mathrm{n}, u, 14} \\ \text { (c) } & M_{5} \ddot{q}_{5}+G_{5}\left(q_{5}\right)=\mathcal{M}_{54}\left(q_{4}, q_{5}\right) \lambda_{\mathrm{n}, u, 45}+\mathcal{M}_{50}\left(q_{5}\right) \lambda_{\mathrm{n}, u, 50}\end{cases}
$$

with $\tau_{1} \in \mathbb{R}^{3}, q_{4} \in \mathbb{R}^{3}$ and $q_{5} \in \mathbb{R}^{3}$ the coordinates (orientation $\theta_{i}$ and CoM position $X_{i}, i=4,5$ ) of bodies $B_{4}$ and $B_{5}$ respectively, $M_{4}$ and $M_{5}$ their mass matrices. The three multipliers are scalars, $G_{i}\left(q_{i}\right)=\frac{d U_{i}}{d q_{i}}\left(q_{i}\right), i=4,5$, for some differentiable functions $U_{i}\left(q_{i}\right)$.

Remark 7 We see that the overall structure of the system (41) is that of a juggling system, with a fully controlled part in (41) (a), and an uncontrolled dynamics in (41) (b) (c). However our goal is not to control (41) (b) (c) through the Lagrange multipliers, but rather to control (41) (a) and view (41) (b) (c) as a disturbance. On the contrary in (25) the objective was to control (25) (a) using the controlled parts of the dynamics (25) (b) and (c). We could apply a similar methodology to (25) and to (41), however these two systems obviously differ from the point of view of their controllability properties of the uncontrolled part with the Lagrange multipliers considered as inputs, named the controllability through impacts in [22, Definition 3] [20, definitions 2, 3, 4]. Controllability through the impacts is intuitively easier to get in (25) than in (41), which motivates our choice for the control strategy. Let us note that [57, Theorem 2] brings an answer for systems as in (25) in the all-linear case, with finite-time stabilization of (25) (a).

The first step is to design $\tau_{1}\left(q_{1}, \dot{q}_{1}, q_{1, d}\right)$ such that the closed-loop system $M_{1}\left(q_{1}\right) \ddot{q}_{1}+C\left(q_{1}, \dot{q}_{1}\right) \dot{q}_{1}+G\left(q_{1}\right)=$ $\tau_{1}\left(q_{1}, \dot{q}_{1}, q_{1, d}\right)$ has the unique fixed point $\left(q_{1}^{\star}, \dot{q}_{1}^{\star}\right)=\left(q_{1, d}, 0\right)$ which is Lyapunov globally asymptotically stable, with a Lyapunov function $V_{1}\left(q_{1}-q_{1, d}, \dot{q}_{1}\right)$. The second step is to analyze the variations of $V_{1}\left(q_{1}-\right.$ $\left.q_{1, d}, \dot{q}_{1}\right)$ at impact times $t_{k}$. In order to guarantee that $\sigma_{V_{1}}\left(t_{k}\right) \triangleq V_{1}\left(t_{k}^{+}\right)-V_{1}\left(t_{k}^{-}\right) \leqslant 0$, one should target preferably a function which mimics the system's total energy, as

$$
V_{1}\left(q_{1}-q_{1, d}, \dot{q}_{1}\right)=\frac{1}{2} \dot{q}_{1}^{T} M_{1}\left(q_{1}\right) \dot{q}_{1}+U\left(q_{1}-q_{1, d}\right),
$$

with $U\left(q_{1}-q_{1, d}\right)$ positive definite and radially unbounded. One possible choice is a PD+gravity compensation input:

$$
\tau_{1}=G\left(q_{1}\right)-K_{p}\left(q_{1}-q_{1, d}\right)-K_{v} \dot{q}_{1},
$$

$K_{p}=K_{p}^{T} \succ 0$ and $K_{v}=K_{v}^{T} \succ 0$, and $U\left(q_{1}-q_{1, d}\right)=\frac{1}{2}\left(q_{1}-q_{1, d}\right)^{T} K_{p}\left(q_{1}-q_{1, d}\right)$. Assume that $C_{1}\left(q_{1}, \dot{q}_{1}\right) \dot{q}_{1}$ is written with the Christoffel's symbols associated with $M_{1}\left(q_{1}\right)$, so that the matrix $\frac{d}{d t} M_{1}\left(q_{1}(t)\right)-2 C_{1}\left(q_{1}, \dot{q}_{1}\right)$ is skew-symmetric [19, Lemma 6.16]. Along the trajectories of (41) (a) (43) with $\lambda_{\mathrm{n}, u, 14}=0$, one has $\dot{V}_{1}\left(q_{1}(t)-q_{1, d}, \dot{q}_{1}(t)\right)=-\dot{q}_{1}^{T} K_{v} \dot{q}_{1}$. The third step is to consider the Lyapunov function candidate

$$
V\left(q_{1}, \dot{q}_{1}, q_{1, d}, q_{4}, q_{5}, \dot{q}_{4}, \dot{q}_{5}\right)=V_{1}\left(q_{1}-q_{1, d}, \dot{q}_{1}\right)+\frac{1}{2} \dot{q}_{4}^{T} M_{4} \dot{q}_{4}+U_{4}\left(q_{4}\right)+\frac{1}{2} \dot{q}_{5}^{T} M_{5} \dot{q}_{5}+U_{5}\left(q_{5}\right) .
$$

During free-motion phases (all multipliers equal to zero) one easily calculates along the trajectories of (41) (43): $\dot{V}(t)=-\dot{q}_{1}^{T} K_{v} \dot{q}_{1}$. At impacts $\sigma_{V}\left(t_{k}\right)=-\frac{1}{2} \frac{1-e_{\mathrm{n}}}{1+e_{\mathrm{n}}} \sigma_{\dot{q}}\left(t_{k}\right)^{T} M\left(q\left(t_{k}\right)\right) \sigma_{\dot{q}}\left(t_{k}\right) \leqslant 0$ for all $e_{\mathrm{n}} \in[0,1]$ [18, Equation (5.61)], because $V(\cdot)$ is simply the sum of the system's kinetic energy and closed-loop potential energy. Let us now investigate persistent contact phases, during which the closed-loop system can be rewritten compactly as the differential inclusion:

$$
\underbrace{M(q)\left(\begin{array}{c}
\ddot{q}_{1} \\
\ddot{q}_{4} \\
\ddot{q}_{5}
\end{array}\right)+C(q, \dot{q})\left(\begin{array}{c}
\dot{q}_{1} \\
\dot{q}_{4} \\
\dot{q}_{5}
\end{array}\right)+\left(\begin{array}{c}
K_{v} \dot{\tilde{q}}_{1}+K_{p} \tilde{q}_{1} \\
G_{4}\left(q_{4}\right) \\
G_{5}\left(q_{5}\right)
\end{array}\right)}_{\text {Closed-loop Lagrangian dynamics (C.L.Lag.Dyn.) }}=\underbrace{\nabla f(q) \lambda_{\mathrm{n}, u} \in-N_{T_{\Phi}(q)}(\dot{q})}_{\text {Static set-valued nonlinearity }},
$$

where the various inertial terms are easily identified from (41) and (5). Differentiating (44) along the trajectories of (45) we get: $\dot{V}(t)=-\dot{q}_{1}^{T} K_{v} \dot{q}_{1}+\dot{q}^{T} \xi$ with $\xi \in-N_{T_{\Phi}(q)}(\dot{q})$. Due to the monotonicity of 
the normal cone mapping and the fact that $0 \in N_{T_{\Phi}(q)}(\dot{q})$, we deduce that $\dot{q}(t)^{T} \xi(t) \leqslant 0$ for all $t \geqslant 0$. Actually if we restrict ourselves to persistent contact phases of motion, and recalling that velocities are right-continuous, we even have the stricter equality $\dot{q}(t)^{T} \xi(t)=0$, see [18, Equation (5.46)]. Thus we have shown that $\dot{V}(t) \leqslant-\dot{q}_{1}(t)^{T} K_{v} \dot{q}_{1}(t)$ outside impact times and $\sigma_{V}\left(t_{k}\right) \leqslant 0$ at impact times. In terms of the differential measure of the function $V(\cdot)$ (which is of local bounded variation provided that velocities also are), we proved that:

$$
d V=-\dot{q}_{1}(t)^{T} K_{v} \dot{q}_{1}(t) d t-\frac{1}{2} \frac{1-e_{\mathrm{n}}}{1+e_{\mathrm{n}}} \sum_{k \geqslant 0} \sigma_{\dot{q}}\left(t_{k}\right)^{T} M\left(q\left(t_{k}\right)\right) \sigma_{\dot{q}}\left(t_{k}\right) \delta_{t_{k}} \leqslant 0,
$$

where the sum is taken over all impact times, including with some abuse of notation possible repeated infinite sequences ${ }^{15}$. Recall that since functions of local bounded variations have a countable set of discontinuities, the sum in the right-hand side of (46) makes sense. The closed-loop system in (45) has the interpretation in Figure 7 (b), as the negative feedback interconnection of two passive subsystems [19]. It is therefore a set-valued Lur'e system [18]. This puts it in a good perspective for stability analysis, and shows that it could be extended to more complex systems with arbitrary number of DoFs and joints with clearance, provided the structure in Figure 7 (b) is preserved.

\subsection{The set of equilibria}

The fourth step is to characterize the fixed points of the closed-loop system. They are solutions of the generalized equation:

$$
\left\{\begin{array}{l}
K_{p}\left(q_{1}^{\star}-q_{1, d}\right)=\mathcal{M}_{14}\left(q_{1}^{\star}, q_{4}^{\star}\right) \lambda_{\mathrm{n}, u, 14}^{\star} \\
G_{4}\left(q_{4}^{\star}\right)=\mathcal{M}_{45}\left(q_{4}, q_{5}\right) \lambda_{\mathrm{n}, u, 45}^{\star}+\mathcal{M}_{41}\left(q_{1}^{\star}, q_{4}^{\star}\right) \lambda_{\mathrm{n}, u, 14}^{\star} \\
G_{5}\left(q_{5}^{\star}\right)=\mathcal{M}_{54}\left(q_{4}^{\star}, q_{5}^{\star}\right) \lambda_{\mathrm{n}, u, 45}^{\star}+\mathcal{M}_{50}\left(q_{5}^{\star}\right) \lambda_{\mathrm{n}, u, 50}^{\star} \\
0 \leqslant f_{14}\left(q_{1}^{\star}, q_{4}^{\star}\right) \perp \lambda_{\mathrm{n}, u, 14}^{\star} \geqslant 0,0 \leqslant f_{45}\left(q_{4}^{\star}, q_{5}^{\star}\right) \perp \lambda_{\mathrm{n}, u, 45}^{\star} \geqslant 0,0 \leqslant f_{50}\left(q_{5}^{\star}\right) \perp \lambda_{\mathrm{n}, u, 50}^{\star} \geqslant 0
\end{array}\right.
$$

for some multipliers $\lambda_{\mathrm{n}, u, 14}^{\star}, \lambda_{\mathrm{n}, u, 45}^{\star}, \lambda_{\mathrm{n}, u, 50}^{\star}$. This can be equivalently rewritten as the inclusion:

$$
0 \in \underbrace{N_{T_{\Phi}\left(q^{\star}\right)}(0)}_{\triangleq_{G\left(q^{\star}\right)}\left(\begin{array}{c}
K_{p}\left(q_{1}^{\star}-q_{1, d}\right) \\
G_{4}\left(q_{4}^{\star}\right) \\
G_{5}\left(q_{5}^{\star}\right)
\end{array}\right)}
$$

where the normal cone inclusion stems from a more general inclusion [18, §B.2.2] and the normal cone $N_{\Phi}\left(q^{\star}\right)$ is defined as in [18, Definition 5.2]. This generalized equation does not possess in general a unique solution but a set $\mathcal{E}$ of solutions (let for instance $G_{4}\left(q_{4}\right)=G_{5}\left(q_{5}\right)=0$, then bodies $\mathbf{B}_{4}$ and $\mathbf{B}_{5}$ have an infinity of equilibrium positions corresponding to positions of journal inside the bearing at joints $J_{4}, J_{5}$ and $\left.J_{6}\right)$. Let us characterize more precisely $\mathcal{E}$. We recall that $\Phi=\{q \in \mathcal{C} \mid f(q) \geqslant 0\} \subseteq \mathbb{R}^{9}$ is the admissible domain, and $\mathcal{I}(q)=\left\{i \in\left\{1, \ldots, m_{u}\right\} \mid f_{i}(q)=0\right\}$ is the set of indices of active constraints which we denote as in section 3 as $f^{\prime}(q)=0$.

Lemma 2 (i) Suppose that $q^{\star} \in \operatorname{int}(\Phi)$, then $q^{\star}$ is an equilibrium if and only if $K_{p}\left(q_{1}^{\star}-q_{1, d}\right)=0$, $G_{4}\left(q_{4}^{\star}\right)=0, G_{5}\left(q_{5}^{\star}\right)=0$. (ii) Suppose that $q^{\star} \in \operatorname{bd}(\Phi)$, then $q^{\star}$ is an equilibrium if and only if there exists $\lambda_{i} \geqslant 0$ such that $G\left(q^{\star}\right)=\sum_{i \in \mathcal{I}\left(q^{\star}\right)} \lambda_{i} \nabla f_{i}\left(q^{\star}\right)$. If the active constraints are independent, this is equivalently rewritten as $G\left(q^{\star}\right) \in \operatorname{ker}\left(I_{9}-\nabla f^{\prime}\left(q^{\star}\right) D\left(q^{\star}\right) \nabla f^{\prime}\left(q^{\star}\right)^{T} M\left(q^{\star}\right)^{-1}\right)$, where $D\left(q^{\star}\right)$ is the Delassus' matrix associated with the active constraints.

\footnotetext{
15 Recall that other, more general impact laws could be used, provided the energetic, kinetic and kinematic constraints are satisfied $[17,47]$.
} 
Proof (i) If $q^{\star} \in \operatorname{int}(\Phi)$ then $T_{\Phi}\left(q^{\star}\right)=\mathbb{R}^{9}$ so $N_{T_{\Phi}\left(q^{\star}\right)}(x)=\{0\}$ for any $x$. (ii) The second part follows from the definition of the normal cone to the tangent cone, see for instance [18, Equation (5.46)]. Adopting the notation of section 3 , the multipliers satisfy $\lambda_{i}=\lambda_{\mathrm{n}, u, i}^{\prime}$. Let us denote $\mathcal{I}\left(q^{\star}\right)=\left\{i_{1}, i_{2}, . ., i_{m_{u}^{\prime}}\right\}$. As we saw in section 3 and using the independency assumption (which guarantees the uniqueness of solution to the contact LCP), we have $\lambda_{\mathrm{n}, u}^{\prime}=-D\left(q^{\star}\right) w\left(q^{\star}, 0\right)$ with the Delassus' matrix

$$
D\left(q^{\star}\right)=\left[\nabla f_{i_{1}}\left(q^{\star}\right), . ., \nabla f_{i_{m_{u}^{\prime}}}\left(q^{\star}\right)\right]^{T} M\left(q^{\star}\right)^{-1}\left[\nabla f_{i_{1}}\left(q^{\star}\right), . ., \nabla f_{i_{m_{u}^{\prime}}}\left(q^{\star}\right)\right],
$$

as the solution of the contact LCP. The vector $w\left(q^{\star}, 0\right)$ is obtained as the vector $w(q, \dot{q}, t)$ in section 3, i.e. $w\left(q^{\star}, 0\right)=-\nabla f^{\prime}\left(q^{\star}\right)^{T} M\left(q^{\star}\right)^{-1} G\left(q^{\star}\right)$. Inserting this expression in the first expression of the equivalence, noting that $\sum_{i \in \mathcal{I}\left(q^{\star}\right)} \lambda_{i} \nabla f_{i}\left(q^{\star}\right)=\nabla f^{\prime}\left(q^{\star}\right) \lambda_{\mathrm{n}, u}^{\prime}$, the result follows.

Lemma 2 (i) concerns equilibria where journals are not in contact with bearings, while (ii) concerns static equilibria where potential forces are balanced by reaction forces. There exists also a panoply of results for generalized equations as in (48) [31,3]. Global existence, uniqueness as well as characterizations of the solution set (convexity, compactness) exist when $\Phi$ is convex, and $G(\cdot)$ satisfies some monotony properties $[31, \S 3.3]$. When $\Phi$ is not convex but finitely represented (as is our case) then local results prevail [31, Proposition 3.3.4]. However [31, Proposition 3.3.4] basically requires that the gradient $\nabla G\left(q^{\star}\right)$ be strictly copositive on some cone, which is rarely satisfied in practice for our systems where $G(q)$ often stems from gravity, or is even zero when gravity acts normally to the system's plane. We can nevertheless see that $q_{1}^{\star}=q_{1, d}$ is an equilibrium if and only if $\mathcal{M}_{14}\left(q_{1, d}, q_{4}^{\star}\right) \lambda_{\mathrm{n}, u, 14}^{\star}=0$, and the generalized equation:

$$
\left\{\begin{array}{l}
G_{4}\left(q_{4}^{\star}\right)=\mathcal{M}_{45}\left(q_{4}, q_{5}\right) \lambda_{\mathrm{n}, u, 45}^{\star} \\
G_{5}\left(q_{5}^{\star}\right)=\mathcal{M}_{54}\left(q_{4}^{\star}, q_{5}^{\star}\right) \lambda_{\mathrm{n}, u, 45}^{\star}+\mathcal{M}_{50}\left(q_{5}^{\star}\right) \lambda_{\mathrm{n}, u, 50}^{\star} \\
0 \leqslant f_{45}\left(q_{4}^{\star}, q_{5}^{\star}\right) \perp \lambda_{\mathrm{n}, u, 45}^{\star} \geqslant 0 \\
0 \leqslant f_{50}\left(q_{5}^{\star}\right) \perp \lambda_{\mathrm{n}, u, 50}^{\star} \geqslant 0
\end{array} \Leftrightarrow 0 \in\left(\begin{array}{l}
G_{4}\left(q_{4}^{\star}\right) \\
G_{5}\left(q_{5}^{\star}\right)
\end{array}\right)+N_{\Phi_{45}}\left(q_{4}^{\star}, q_{5}^{\star}\right)+N_{\Phi_{50}}\left(q_{5}^{\star}\right)\right.
$$

is solvable, with $\Phi_{45}=\left\{(x, y) \in \mathbb{R}^{3} \times \mathbb{R}^{3} \mid f_{45}(x, y) \geqslant 0\right\}$ and $\Phi_{50}=\left\{x \in \mathbb{R}^{3} \mid f_{50}(x) \geqslant 0\right\}$. The generalized equation (50) is solvable when $G_{4}\left(q_{4}^{\star}\right)=0$ and $G_{5}\left(q_{5}^{\star}\right)=0$. We conjecture that this is also the case for gravity potentials because the system can always find a static equilibrium inside the revolute joints with clearance. However a better analysis of $\mathcal{E}$ requires the expression of the constraints gradients, i.e. of the normal cones in the right-hand side of $(50)$.

\subsection{Stability via Krasovskii-LaSalle invariance principle}

The fifth step is to characterize some invariant sets. Let us denote $x \in \mathbb{R}^{9}$ the closed-loop system's state vector. From $d V \leqslant 0$ and the fact that $V(\cdot)$ is of local bounded variation, we deduce that $V(\cdot)$ is nonincreasing and from [47, Proposition 6.5] the set $\Omega_{0}=\left\{x \in \mathbb{R}^{9} \mid V(x) \leqslant V(x(0))\right\}$ is positively invariant with respect to the closed-loop dynamics. Let us introduce the set:

$$
\begin{aligned}
\mathcal{Z} & =\left\{x \in \Omega_{0} \mid 0=d V(x), x \text { solution of the closed-loop system }\right\} \\
& =\left\{x \in \Omega_{0} \mid \dot{q}_{1}(t)=0 \text { for all } t \geqslant 0, \dot{q}\left(t_{k}^{+}\right)=\dot{q}\left(t_{k}^{-}\right) \text {for all } t_{k}, k \geqslant 0, \text { if } e_{\mathrm{n}} \in[0,1)\right\} .
\end{aligned}
$$

Let $e_{\mathrm{n}} \in[0,1)$. The largest invariant set $\mathcal{V}$ in $\mathcal{Z}$ is contained in the set of continuous-time solutions of

$$
\left\{\begin{array}{l}
\left(\text { a) } K_{p}\left(q_{1}-q_{1, d}\right)=\mathcal{M}_{14}\left(q_{1}, q_{4}(t)\right) \lambda_{\mathrm{n}, u, 14}(t)\right. \\
\left(\text { b) } M_{4} \ddot{q}_{4}(t)+G_{4}\left(q_{4}(t)\right)=\mathcal{M}_{45}\left(q_{4}(t), q_{5}(t)\right) \lambda_{\mathrm{n}, u, 45}(t)+\mathcal{M}_{41}\left(q_{1}, q_{4}(t)\right) \lambda_{\mathrm{n}, u, 14}(t)\right. \\
(c) M_{5} \ddot{q}_{5}(t)+G_{5}\left(q_{5}(t)\right)=\mathcal{M}_{54}\left(q_{4}(t), q_{5}(t)\right) \lambda_{\mathrm{n}, u, 45}(t)+\mathcal{M}_{50}\left(q_{5}(t)\right) \lambda_{\mathrm{n}, u, 50}(t) \\
(d) 0 \leqslant f_{14}\left(q_{1}(t), q_{4}(t)\right) \perp \lambda_{\mathrm{n}, u, 14}(t) \geqslant 0,0 \leqslant f_{45}\left(q_{4}(t), q_{5}(t)\right) \perp \lambda_{\mathrm{n}, u, 45}(t) \geqslant 0, \\
\quad 0 \leqslant f_{50}\left(q_{5}(t)\right) \perp \lambda_{\mathrm{n}, u, 50}(t) \geqslant 0,
\end{array}\right.
$$

for some constant $q_{1}$. Clearly $\mathcal{E} \subseteq \mathcal{V}$. From (52) (a) we deduce that both $q_{4}$ and $\lambda_{\mathrm{n}, u, 14}$ are constant. Thus (52) (a) becomes $G_{4}\left(q_{4}\right)=\mathcal{M}_{45}\left(q_{4}, q_{5}(t)\right) \lambda_{\mathrm{n}, u, 45}(t)+\mathcal{M}_{41}\left(q_{1}, q_{4}\right) \lambda_{\mathrm{n}, u, 14}$, from which we infer that $q_{5}$ and $\lambda_{\mathrm{n}, u, 45}$ are constant. Using (52) (c) it follows that $\lambda_{\mathrm{n}, u, 50}$ is constant also. Therefore we conclude that $\mathcal{V}=\mathcal{E}$. 
Proposition 3 Consider the six-bar system (41) with $\tau_{1}=G\left(q_{1}\right)-K_{p}\left(q_{1}-q_{1, d}\right)-K_{v} \dot{q}_{1}, e_{\mathrm{n}} \in[0,1)$ and $M_{1}\left(q_{1}\right) \succ 0$. (i) Let $U_{4}\left(q_{4}\right) \geqslant 0$ and $U_{5}\left(q_{5}\right) \geqslant 0$. The functions $q_{1}(\cdot)-q_{1, d}, \dot{q}_{1}(\cdot), \dot{q}_{4}(\cdot)$ and $\dot{q}_{5}(\cdot)$ are bounded for any initial condition, i.e. there exist $c_{i}\left(V(x(0)), c_{i}(0)=0,1 \leqslant i \leqslant 4\right.$ such that $\left\|q_{1}(t)\right\| \leqslant c_{1}(V(x(0))$, $\left\|\dot{q}_{1}(t)\right\| \leqslant c_{2}\left(V(x(0)),\left\|\dot{q}_{4}(t)\right\| \leqslant c_{3}\left(V(x(0)),\left\|\dot{q}_{5}(t)\right\| \leqslant c_{4}(V(x(0))\right.\right.$ for all $t \geqslant 0$. (ii) The coordinates $q_{4}(t)$ and $q_{5}(t)$ are bounded for all $t \geqslant 0$. (iii) Assume that trajectories depend continuously on the initial data. Let $U_{4}\left(q_{4}\right)$ and $U_{5}\left(q_{5}\right)$ be continuous and bounded from below. Then (i) holds and every solution with $x(0) \in \Omega_{0}$ converges asymptotically to the set of equilibrium points $\mathcal{E}$.

Proof (i) We have $\lambda_{\min }\left(M_{1}\left(q_{1}\right)\right)\left\|\dot{q}_{1}(t)\right\|^{2} \leqslant \dot{q}_{1}(t)^{T} M_{1}\left(q_{1}(t)\right) \dot{q}_{1}(t) \leqslant V(t) \leqslant V(0)$ for all $t \geqslant 0$, which implies that $\dot{q}_{1}(t)$ is bounded. The same applies to the other variables with a similar proof. (ii) Let $X_{i}$ denote the CoM $G_{i}$ coordinates in an absolute coordinate frame, same for $X_{i+1}$, see Figure 1 (b). Clearly $\left\|X_{i}\right\| \leqslant M_{i}<+\infty$ for some $M_{i}$, implies that $\left\|X_{i+1}\right\| \leqslant M_{i+1}<+\infty$ for some $M_{i+1}$, due to the geometry of revolute joints. In our case the boundedness of $q_{1}$ implies that of $X_{3}$, thus of $X_{4}$ and then of $X_{5}$. The orientation angles $\theta_{4}$ and $\theta_{5}$ are bounded as well due to the kinematic closed-loop in the six-bar mechanism. (iii) The result follows from [47, Theorem 6.31] and the fact that $V(\cdot)$ can be modified using $U_{4}\left(q_{4}\right)-U_{4, \min } \geqslant 0$ and $U_{5}\left(q_{5}\right)-U_{5, \min } \geqslant 0$.

The assumption that trajectories are continuous in the initia data, secures that Krasovskii-LaSalle invariance results apply [47]. We do not check that this is the case for our system, because this requires the calculation of kinetic angles between constraints [18, §5.2.4], and may happen only in case of multiple impacts. Proposition 3 guarantees that if $V(x(0))$ is small enough, then $q_{1}$ remains close to $q_{1, d}$. We may now use a kinematic property of the six-bar system, that stems from the fact that the clearance-free system has three DoFs. We infer that there exists functions $g_{4}(\cdot)$ and $g_{5}(\cdot)$ such that $\theta_{4}=g_{4}\left(\theta_{1}, \theta_{2}, \theta_{3}\right)$ and $\theta_{5}=g_{5}\left(\theta_{1}, \theta_{2}, \theta_{3}\right)$, and that these functions are bounded (see explicit calculations in [4, Appendix A] for a four-bar kinematic chain). In the presence of mechanical play boundedness of $q_{4}$ and $q_{5}$ still holds, indeed the subsystem $S_{2}$ has limited range of motion for finite clearance values and with the rest of the state variables being bounded.

Remark 8 This analysis shows that a feedback controller designed from the dynamics with clearance, is robust with respect to joint-clearance dynamical effects and it does not need measurements of the state variables of bodies $\mathbf{B}_{4}$ and $\mathbf{B}_{5}$, contrarily to controllers for juggling systems. It does not need the knowledge of the CoRs neither, but only the dissipativity at impacts (loss of kinetic energy). The price to pay is that the trajectories may not always converge to the desired fixed point. The robustness of a controller designed for the clearance-free system (5) is another matter.

Remark 9 We have not incorporated the kinematic properties of the analysed mechanism, like intrinsic angular displacement bounds in some systems like four-bar rocker-rocker and crank-rocker mechanisms. This is going to affect the set $\mathcal{E}$ of equilibria, especially the solvability of (50).

Remark 10 The controller $\tau_{1}$ in (43) guarantees that $\dot{V}_{1}$ is semi-negative definite along solutions derivatives outside impacts. Other controllers could be chosen. For instance passivity-based controllers like the Slotine and Li one $[19, \S 7.3 .4]$ with $\tau_{1}=-M_{1}\left(q_{1}\right) \Lambda \dot{q}_{1}-C_{1}\left(q_{1}, \dot{q}_{1}\right) \Lambda\left(q_{1}-q_{1, d}\right)+G_{1}\left(q_{1}\right)-K s_{1}$, $s_{1}=\dot{q}_{1}+\Lambda\left(q_{1}-q_{1, d}\right), K=K^{T} \succ 0, \Lambda=\Lambda^{T} \succ 0$, yields a Lyapunov function (for the unconstrained subsystem (41) (a)) $V_{1}\left(q_{1}-q_{1, d}, \dot{q}_{1}\right)=\frac{1}{2} s_{1}^{T} M_{1}\left(q_{1}\right) s_{1}+2\left(q_{1}-q_{1, d}\right)^{T} \Lambda K\left(q_{1}-q_{1, d}\right)$, which satisfies along the unconstrained system's trajectories $\dot{V}_{1}(t)=-s_{1}^{T} K s_{1}+2\left(q_{1}-q_{1, d}\right)^{T} \Lambda K \dot{q}_{1}=-\dot{q}_{1}^{T} K \dot{q}_{1}-\left(q_{1}-\right.$ $\left.q_{1, d}\right)^{T} \Lambda K \Lambda\left(q_{1}-q_{1, d}\right)$ [79], which is a stronger property than the one obtained with (42) (43) (44). However at impacts $\sigma_{V}\left(t_{k}\right)=\underbrace{-\frac{1}{2} \frac{1-e_{\mathrm{n}}}{1+e_{\mathrm{n}}} \sigma_{\dot{q}}\left(t_{k}\right)^{T} M\left(q\left(t_{k}\right)\right) \sigma_{\dot{q}}\left(t_{k}\right)}_{\leqslant 0 \text { for all } e_{\mathrm{n}} \in[0,1]}+\left(q_{1}\left(t_{k}\right)-q_{1, d}\right)^{T} \Lambda \underbrace{M_{1}\left(q_{1}\left(t_{k}\right)\right) \sigma_{\dot{q}_{1}}\left(t_{k}\right)}_{=\mathcal{M}_{14}\left(q_{1}, q_{4}\right) p_{\mathrm{n}, u, 14}\left(t_{k}\right)}$, where $p_{\mathrm{n}, u, 14}\left(t_{k}\right)$ is the density of $\lambda_{\mathrm{n}, u, 14}$ at $t_{k}$ (the impulse). How to guarantee the non-positivity of the second term is not obvious. During persistent contact phases, the following equality holds: $\dot{V}(t)=$ $-\dot{q}_{1}^{T} K \dot{q}_{1}-\left(q_{1}-q_{1, d}\right)^{T} \Lambda K \Lambda\left(q_{1}-q_{1, d}\right)+\dot{q}^{T} \xi+\left(q_{1}-q_{1, d}\right)^{T} \Lambda \mathcal{M}_{14}\left(q_{1}, q_{4}\right) \lambda_{\mathrm{n}, u, 14}, \xi \in-N_{T_{\Phi}(q)}(\dot{q})$. Again 
the sign of $\left(q_{1}-q_{1, d}\right)^{T} \Lambda \mathcal{M}_{14}\left(q_{1}, q_{4}\right) \lambda_{\mathrm{n}, u, 14}$ is not guaranteed. Thus we get instead of (46):

$$
\begin{aligned}
d V & \leqslant \underbrace{-\left[\dot{q}_{1}(t)^{T} K \dot{q}_{1}(t)+\left(q_{1}(t)-q_{1, d}\right)^{T} \Lambda K \Lambda\left(q_{1}(t)-q_{1, d}\right)\right]}_{\triangleq \dot{V}_{1}^{\text {free }}(t) \leqslant 0} d t-\frac{1}{2} \frac{1-e_{\mathrm{n}}}{1+e_{\mathrm{n}}} \sum_{k \geqslant 0} \sigma_{\dot{q}}\left(t_{k}\right)^{T} M\left(q\left(t_{k}\right)\right) \sigma_{\dot{q}}\left(t_{k}\right) \delta_{t_{k}} \\
& +\left(q_{1}-q_{1, d}\right)^{T} \Lambda \mathcal{M}_{14}\left(q_{1}, q_{4}\right) \lambda_{\mathrm{n}, u, 14}(t) d t+\sum_{k \geqslant 0}\left(q_{1}\left(t_{k}\right)-q_{1, d}\right)^{T} \Lambda M_{1}\left(q_{1}\left(t_{k}\right)\right) \sigma_{\dot{q}_{1}}\left(t_{k}\right) \delta_{t_{k}} .
\end{aligned}
$$

A possible path could be to guarantee that the error $q_{1}-q_{1, d}$ is very small each time contact or impact occurs, taking advantage of the exponential decrease of $\dot{q}_{1}(t)$ and $\left(q_{1}(t)-q_{1, d}\right)$ when journals and bearings are not in contact. Another path is to analyse under which conditions the system spends enough time in unconstrained motion (a dwell-time condition), so that the exponential decrease due to $\dot{V}_{1}^{\text {free }}(t)$ in (53) compensates for possibly positive variations in $V(\cdot)$. In $[21,58]$ this problem is avoided because the controller is designed after a change of generalized coordinates and a suitable adaptation of the desired trajectory, which secure that the unsigned terms vanish. However the control strategy analysed in $[21,58]$ does not seem applicable to systems with joint clearance in general, because it applies to fully-actuated systems $(p=n$ in (1)), it relies on a specific sequence of phases of motion that is taylored to robotic manipulators' tasks [18, Equations (8.4), (8.37)-(8.40)], it needs the measurement of the whole state (position and velocity), and finally it is designed after a generalized coordinate change $z=Z(q)$ which transforms the constraints $f_{i}(q) \geqslant 0$ into $z_{i} \geqslant 0$ in the new coordinates. However in the $z$ coordinates the specific structure (41) on which our analysis relies, no longer exists (the same holds for (25) and (26) for which the juggling system structure should be preserved). We may also add that in some cases, the transformation $z=Z(q)$ is not invertible. For the one-DoF prismatic joint in Figure 2, working in such quasi-coordinates means setting $z=\left(\begin{array}{cc}-1 & 1 \\ 1 & -1\end{array}\right) q+\left(\begin{array}{c}-l \\ L-l\end{array}\right)$ which is not invertible.

We have led the analysis for the six-bar system in Figure 7 (a), but it can easily be extended to other systems, as long as some basic requirements are fulfilled which preserve the controlled system's structure, see Figure 7 (b). For instance the four-bar system in Figure 6 (b), where $\theta_{m s}$ is the motorshaft angle, may be controlled applying a feedback torque at $J_{11}$ with measurement of $\theta_{m s}$ and $\dot{\theta}_{m s}$.

Remark 11 Non-collocation of the controller introduces an equivalent disturbance in the closed-loop system, deteriorating its performance further. The same applies for controllers designed for the clearance-free system (7), and implemented on the mechanism with joint clearances.

\subsection{Non-collocated control}

Let us consider the system in Figure 5 (a), with joints $J_{1}, J_{2}, J_{3}$ actuated with $\tau_{1}$ in (41) (a) and (43). In the clearance-free case, the controller is non-collocated if the feedback is calculated from measurements of $\theta_{1}, \theta_{4}, \theta_{5}$ and their derivatives. Consider the two subsystems $S_{1}=\left(\mathbf{B}_{1}, \mathbf{B}_{2}, \mathbf{B}_{3}\right)$ and $S_{2}=\left(\mathbf{B}_{4}, \mathbf{B}_{5}\right)$, and connect them with the revolute joint $J_{4}$ which produces two bilateral constraints $h_{i}\left(\theta_{1}, \theta_{2}, \theta_{3}, \theta_{4}, \theta_{5}\right)=0, i=1,2$. Inversion of the problem $h_{1}=h_{2}=0$ provides expressions like $\theta_{2}=g_{2}\left(\theta_{1}, \theta_{4}, \theta_{5}\right)$ and $\theta_{3}=g_{3}\left(\theta_{1}, \theta_{4}, \theta_{5}\right)$ for some $g_{2}(\cdot)$ and $g_{3}(\cdot)^{16}$. Replacing $\theta_{2}, \dot{\theta}_{2}, \theta_{3}, \dot{\theta}_{3}$ by $g_{2}\left(\theta_{1}, \theta_{4}, \theta_{5}\right)$, $\dot{g}_{2}\left(\theta_{1}, \theta_{4}, \theta_{5}, \dot{\theta}_{1}, \dot{\theta}_{4}, \dot{\theta}_{5}\right), g_{3}\left(\theta_{1}, \theta_{4}, \theta_{5}\right)$, and $\dot{g}_{3}\left(\theta_{1}, \theta_{4}, \theta_{5}, \dot{\theta}_{1}, \dot{\theta}_{4}, \dot{\theta}_{5}\right)$ respectively, in the feedback $\tau_{1}$, produces a non-collocated input. In the ideal case both collocated and non-collocated inputs are equivalent. Assume now that there is a clearance at $J_{5}$. Then $g_{2}$ and $g_{3}$ are only approximate estimations of $\theta_{2}$ and $\theta_{3}$, since the bearing and journal centers no longer match at all times. We obtain the approximation $q_{1}^{a p p} \triangleq\left(\theta_{1}, g_{2}\left(\theta_{1}, \theta_{4}, \theta_{5}\right), g_{3}\left(\theta_{1}, \theta_{4}, \theta_{5}\right)\right)^{T}$, and (41) (a) becomes in closed-loop:

$$
\begin{aligned}
& M_{1} \ddot{q}_{1}+C_{1}\left(q_{1}, \dot{q}_{1}\right) \dot{q}_{1}+G\left(q_{1}\right)-G\left(q_{1}^{a p p}\right)+K_{p}\left(q_{1}^{a p p}-q_{1, d}\right)+K_{v} \dot{q}_{1}^{a p p}=\mathcal{M}_{14}\left(q_{1}, q_{4}\right) \lambda_{\mathrm{n}, u, 14} \\
\Leftrightarrow & M_{1}\left(q_{1}\right) \ddot{q}_{1}+C_{1}\left(q_{1}, \dot{q}_{1}\right) \dot{q}_{1}+K_{p}\left(q_{1}-q_{1, d}\right)+K_{v} \dot{q}_{1}=D_{\mathrm{eq}}\left(q_{1}, \dot{q}_{1}, \theta_{4}, \theta_{5}, \dot{\theta}_{4}, \dot{\theta}_{5}\right)+\mathcal{M}_{14}\left(q_{1}, q_{4}\right) \lambda_{\mathrm{n}, u, 14}
\end{aligned}
$$

\footnotetext{
16 We do not detail the conditions under which these two functions may be obtained because this is not the object of our analysis.
} 
with the equivalent disturbance

$$
D_{\text {eq }}\left(q_{1}, \dot{q}_{1}, \theta_{4}, \theta_{5}, \dot{\theta}_{4}, \dot{\theta_{5}}\right) \triangleq G\left(q_{1}^{a p p}\right)-G\left(q_{1}\right)+K_{p}\left(q_{1}-q_{1}^{a p p}\right)+K_{v}\left(\dot{q}_{1}-\dot{q}_{1}^{a p p}\right)
$$

It is not difficult to show that (41) (a) with $\tau_{1}$ in (43) defines a passive operator $\mathcal{M}_{14}\left(q_{1}, q_{4}\right) \lambda_{\mathrm{n}, u, 14} \mapsto \dot{q}_{1}$, and that this allows us to recast the closed-loop dynamics (41) (43), including the complementarity conditions and the impact law, into passive systems made of the negative feedback interconnection of two passive subsystems, see [15] [18, Fig. 7.6] [19, Fig. 6.7]. With (54) the passivity property is lost and stability analysis is less easy due to the disturbance in (55) (it is a well-known fact that non-collocation may even create instability). In $[53, \S 3.1,3.2]$ it is shown on the one-DoF prismatic joint in Figure 2 (b) that non-collocation of a proportional-derivative controller $\tau_{1}$ introduces limit cycles in the closed-loop system. Existence with viability of trajectories, and a stability criterion of the impact Poincaré mapping are given in $[53, \S 3.2]$, using classical techniques for the analysis of periodic trajectories in vibro-impact systems $[9][18, \S 7.3]$.

\subsection{Controller designed for the clearance-free system (7) and applied to the system with clearance}

Let us now assume that the controller has been designed for the clearance-free system in (7), supposed to be fully actuated (i.e. $\left.E_{w c}\left(q_{w c}\right)=I_{n_{w c}}\right)$, and is applied to the system with joint-clearance (1). We clearly get an under-actuated system with $p=n_{w c}<n$. The problem is different from that analysed in section 5 , where $\tau_{1}$ in (43) is designed to stabilize the unconstrained actuated dynamics (41) (a). In our case we can choose $q_{w c}=q_{1}$. Instead of $(43)$ we now set $\tau=g_{w c}\left(q_{1}\right)-K_{p}\left(q_{1}-q_{1, d}\right)-K_{v} \dot{q}_{1}$. However $g_{w c}\left(q_{1}\right) \neq G_{1}\left(q_{1}\right)$, since $g_{w c}(\cdot)$ takes into account the gravity forces from all subsystems $S_{1}, S_{2}$ and $S_{3}$, while $G_{1}(\cdot)$ concerns only $S_{1}$. Thus the equivalent disturbance is simpler than (55) with $D_{\text {eq }}\left(q_{1}\right)=g_{w c}\left(q_{1}\right)-G_{1}\left(q_{1}\right)$, which is globally bounded for the six-bar system. The fixed-points of the unconstrained closed-loop system (41) (a) are $\left(q_{1}^{\star}, 0\right)$, with $q_{1}^{\star}$ solution (possibly non-unique) of $K_{p}\left(q_{1}^{\star}-q_{1, d}\right)=D_{\text {eq }}\left(q_{1}^{\star}\right)$, while (48) is modified accordingly. We obtain along closed-loop trajectories $\dot{V}_{1}(t)=-\dot{q}_{1}^{T} K_{v} \dot{q}_{1}+\dot{q}_{1}^{T} D_{\text {eq }}\left(q_{1}\right)$. It is possible to redo a similar analysis as the one of section 5 , however the closed-loop system performance with such an input will be less good than with (43).

Remark 12 We may consider also the behaviour of a closed-loop system, when the feedback control is designed for the system with joint-clearance and dimension $n(=p)$, and is applied to the clearance-free system in $(7)$ with $\operatorname{dim}\left(q_{w c}\right)<n$. The clearance-free system is over-actuated and control actions have to be suitably distributed among the redundant controllers.

\section{Trajectory tracking}

Two main control analysis may be led: study the robustness of controllers designed for the clearance-free system (7) when applied to (1) [4], or design specific controllers for (1). In some applications it is sometimes sufficient to use simple controllers for stabilization and change the desired equilibrium (constant $q_{1, d}$ in section 5) according to some exogenous rule. However the Control literature provides several nice solutions for the trajectory tracking problem of Lagrangian systems, and it is worth investigating how they may be adapted to systems with joint clearance.

\subsection{Incorporating clearance effects in the control design}

This section is devoted to the extension of results in section 5 for trajectory tracking. As alluded to above, the results in $[21,58]$ apply to systems with $m_{b}=0$ and $p=n$, measurement of $q$ and $\dot{q}$. Their application to systems with clearances is usually impossible, as it would imply full actuation of all the open system's DoFs (consider for instance the six-bar system of section 4: full actuation of subsystem $S_{2}$ 
in (25) (a) requires an input $\tau_{2} \in \mathbb{R}^{3}$ ). There is however one case in which tracking controllers may be used: consider the six-bar system in Figure 5 (a) with a clearance at joint $J_{3}$. The system may be seen as two subsystems $S_{1}=\left(\mathbf{B}_{1}, \mathbf{B}_{2}\right)$ with two DoFs, $S_{2}=\left(\mathbf{B}_{3}, \mathbf{B}_{4}, \mathbf{B}_{5}\right)$ with three DoFs. If joints $J_{1}, J_{2}, J_{4}$, $J_{5}$ and $J_{6}$ are actuated, the dynamics may be written as:

$$
\left\{\begin{array}{l}
M_{1}\left(q_{1}\right) \ddot{q}_{1}+C_{1}\left(q_{1}, \dot{q}_{1}\right) \dot{q}_{1}+G_{1}\left(q_{1}\right)=\tau_{1}+\mathcal{M}_{12}\left(q_{1}, q_{2}\right) \lambda_{\mathrm{n}, u, 12} \\
M_{2}\left(q_{2}\right) \ddot{q}_{2}+C_{2}\left(q_{2}, \dot{q}_{2}\right) \dot{q}_{2}+G_{2}\left(q_{2}\right)=\tau_{2}+\mathcal{M}_{21}\left(q_{1}, q_{2}\right) \lambda_{\mathrm{n}, u, 12} \\
0 \leqslant f_{12}\left(q_{1}, q_{2}\right) \perp \lambda_{\mathrm{n}, u, 12} \geqslant 0 \\
\text { Impact law in (4). }
\end{array}\right.
$$

This perfectly fits with the Lagrangian systems analysed in $[21,58]$ with $\tau_{1} \in \mathbb{R}^{2}$ and $\tau_{2} \in \mathbb{R}^{3}$. However the direct application of these results relies on a specific succession of modes of motion (see [18, §8.1.1.1 and 8.3.1.1]) which is not appropriate for systems with mechanical play. Let us analyze the extension of collocated stabilization via PD+ controller, using the scheme proposed in [66] when applied to (56). Let $\tilde{q}=q-q_{d}, \tau=\left(\tau_{1}^{T}, \tau_{2}^{T}\right)^{T}, q=\left(q_{1}^{T}, q_{2}^{T}\right)^{T}, M(q)=\operatorname{diag}\left(M_{1}\left(q_{1}\right), M_{2}\left(q_{2}\right)\right), C(q, \dot{q}) \dot{q}+G(q)=$ $\left(\begin{array}{l}C_{1}\left(q_{1}, \dot{q}_{1}\right) \dot{q}_{1}+G_{1}\left(q_{1}\right) \\ C_{2}\left(q_{2}, \dot{q}_{2}\right) \dot{q}_{2}+G_{2}\left(q_{2}\right)\end{array}\right)$. It is still assumed that Christoffel's symbols are used so that the skew-symmetry property of $\frac{d}{d t} M(q(t))-2 C(q(t), \dot{q}(t))$ is satisfied [19, Lemma 6.16]. The controller is given by:

$$
\tau=M(q) \ddot{q}_{d}+C(q, \dot{q}) \dot{q}_{d}+G(q)-K_{v} \dot{\tilde{q}}-K_{p} \tilde{q}
$$

with $K_{p}=K_{p}^{T} \succ 0, K_{v}=K_{v}^{T} \succ 0$. It gives rise to the closed-loop system

$$
M(q) \ddot{\tilde{q}}+C(q, \dot{q}) \dot{\tilde{q}}+K_{v} \dot{\tilde{q}}+K_{p} \tilde{q}=\nabla f(q) \lambda_{\mathrm{n}, u} \in-N_{T_{\Phi}(q)}(w(t)) .
$$

The Lyapunov function is:

$$
V(\tilde{q}, \dot{\tilde{q}})=\frac{1}{2} \dot{\tilde{q}}^{T} M(q) \dot{\tilde{q}}+\frac{1}{2} \tilde{q}^{T} K_{p} \tilde{q},
$$

and its derivative along (58), outside impacts (i.e. when $\left.N_{T_{\Phi}(q)}(w(t))=\{0\}\right)$, is $\dot{V}(t)=-\dot{\tilde{q}}(t)^{T} K_{v} \dot{\tilde{q}}(t)$. Notice the similarity with (42) (43) (44), however this time the closed-loop system is time-varying and invariance results cannot be applied for the convergence towards the equilibrium points, as done in Proposition 3 . The jump of $V(\cdot)$ in (59) at impact times is given by:

$$
\sigma_{V}\left(t_{k}\right)=-\frac{1}{2} \frac{1-e_{\mathrm{n}}}{1+e_{\mathrm{n}}} \sigma_{\dot{q}}\left(t_{k}\right)^{T} M\left(q\left(t_{k}\right)\right) \sigma_{\dot{q}}\left(t_{k}\right)-\dot{q}_{d}\left(t_{k}\right)^{T} M\left(q\left(t_{k}\right)\right) \sigma_{\dot{q}}\left(t_{k}\right) .
$$

where $\sigma_{\dot{q}}\left(t_{k}\right) \triangleq \dot{q}\left(t_{k}^{+}\right)-\dot{q}\left(t_{k}^{-}\right)$, we supposed that $\dot{q}_{d}$ is continuous at $t_{k}$, and we used the kinetic energy loss as in [18, Equ. (5.61)]. The first term in the right-hand side of (60) is non positive (complete system's kinetic energy loss). Using the impact dynamics the second term may be rewritten as $\dot{q}_{d}\left(t_{k}\right)^{T} \xi$ with $\xi \in-N_{T_{\Phi}\left(q\left(t_{k}\right)\right)}\left(w\left(t_{k}\right)\right), w\left(t_{k}\right)=\frac{\dot{q}\left(t_{k}^{+}\right)+e_{\mathrm{n}} \dot{q}\left(t_{k}^{-}\right)}{1+e_{\mathrm{n}}}$. The objective is to guarantee that $\dot{q}_{d}\left(t_{k}\right)^{T} \xi \geqslant 0$, which is equivalent to $\dot{q}_{d}\left(t_{k}\right) \in\left(N_{T_{\Phi}\left(q\left(t_{k}\right)\right)}\left(w\left(t_{k}\right)\right)\right)^{\circ}$, where $(\cdot)^{\circ}$ denotes the polar cone of convex analysis. The polar cone to the normal cone $N_{K}(x)$ to a non empty convex closed set $K$ at $x$ being by definition the tangent cone $T_{K}(x)$ [39, Proposition 5.2.4], we obtain the fundamental condition on the desired trajectory derivative at impact times:

$$
\dot{q}_{d}\left(t_{k}\right) \in T_{T_{\Phi}\left(q\left(t_{k}\right)\right)}\left(w\left(t_{k}\right)\right) .
$$

Following similar developments as in $[18, \S 5.2 .2 .2$, B.2.2], we deduce that (61) is equivalently formulated as $\dot{q}_{d}\left(t_{k}\right)^{T} \nabla f_{i}\left(q\left(t_{k}\right)\right) \geqslant 0$ for all $i \in \mathcal{J}\left(q\left(t_{k}\right)\right)$ and $w\left(t_{k}\right)^{T} \nabla f_{i}\left(q\left(t_{k}\right)\right)=0$. After that, elementary convex analysis yields $w\left(t_{k}\right)=\operatorname{proj}_{M\left(q\left(t_{k}\right)\right)}\left[T_{\Phi}\left(q\left(t_{k}\right)\right) ; \dot{q}\left(t_{k}^{-}\right)\right][18$, Equ. (5.61)] (the orthogonal projection in the metric defined by $\left.M\left(q\left(t_{k}\right)\right)\right)$. It is therefore possible to guarantee (61) from the knowledge of pre-impact velocities and positions. We note that $\dot{q}_{d}=0$ always is a solution of (61) since the right-hand side is a cone. Let us analyse now persistent contact phases. The derivative of the Lyapunov function candidate in (59) is calculated along the closed-loop system's trajectories as:

$$
\dot{V}(t)=-\dot{\tilde{q}}(t)^{T} K_{v} \dot{\tilde{q}}(t)+\dot{q}^{T}(t) \xi-\dot{q}_{d}(t)^{T} \xi, \quad \xi \in-N_{T_{\Phi}(q(t))}(\dot{q}(t)),
$$


because $w(t)=\dot{q}(t)$ in this case. The second term in the right-hand side of (62) is non-positive (for the same reasons as in section 5 after $(44))$, and the third term is negative for all $\xi \in-N_{T_{\Phi}(q(t))}(\dot{q}(t))$ if and only if:

$$
\dot{q}_{d}(t) \in T_{T_{\Phi}(q(t))}(\dot{q}(t))
$$

which is the counterpart of $(61)^{17}$. We can thus obtain the counterpart of (46) for tracking, assuming that both (61) and (63) hold at all times:

$$
d V \leqslant-\dot{\tilde{q}}(t)^{T} K_{v} \dot{\tilde{q}}(t) d t-\frac{1}{2} \frac{1-e_{\mathrm{n}}}{1+e_{\mathrm{n}}} \sum_{k \geqslant 0} \sigma_{\dot{q}}\left(t_{k}\right)^{T} M\left(q\left(t_{k}\right)\right) \sigma_{\dot{q}}\left(t_{k}\right) \delta_{t_{k}} \leqslant 0 .
$$

Proposition 4 Consider the system (56) with the controller (57), and assume that conditions (61) and (63) hold at all $t \geqslant 0$. Then $\tilde{q}(\cdot)$ and $\dot{\tilde{q}}(\cdot)$ are bounded for any initial condition. More precisely $\|\tilde{q}(t)\| \leqslant$ $\sqrt{\frac{V(0)}{\lambda_{\min }\left(K_{p}\right)}}$ and $\|\dot{\tilde{q}}\| \leqslant \sqrt{\frac{V(0)}{\lambda_{\min }(M(q))}}$.

Proof Velocities are of local bounded variations, and so is $V(\cdot)$ in (59). Hence from (64), $V(\cdot)$ is nonincreasing and the result follows.

Some comments arise:

1. Going further in the stability analysis requires the extension of invariance principle to time-varying systems, as done in [66] for the unconstrained case. This is outside the scope of this paper but is certainly worth investigating. As alluded to above, the practical application of this control strategy with conditions (61) and (63), requires the knowledge of $q$ and $\dot{q}$. In theory this is sufficient to detect contact and impact times. Suppose that condition (61) is not respected, then using (60) we infer that (64) becomes:

$$
d V \leqslant-\dot{\tilde{q}}(t)^{T} K_{v} \dot{\tilde{q}}(t) d t-\sum_{k \geqslant 0} \underbrace{\left(\frac{1}{2} \frac{1-e_{\mathrm{n}}}{1+e_{\mathrm{n}}} \lambda_{\min }(M(q))\left\|\sigma_{\dot{q}}\left(t_{k}\right)\right\|-\left\|\dot{q}_{d, \max }\right\| \lambda_{\max }(M(q))\right)}_{\triangleq_{r\left(e_{\mathrm{n}},\left\|\dot{q}_{d, \max }\right\|\right)}}\left\|\sigma_{\dot{q}}\left(t_{k}\right)\right\| \delta_{t_{k}} \leqslant 0 .
$$

where $\left\|\dot{q}_{d, \max }\right\|=\max _{t \geqslant 0}\left\|\dot{q}_{d}(t)\right\|$. We have $r\left(e_{\mathrm{n}},\left\|\dot{q}_{d, \max }\right\|\right) \geqslant 0$ for $\left\|\dot{q}_{d, \text { max }}\right\|$ small enough and $e_{\mathrm{n}}<1$. This indicates that small desired velocities combined with enough dissipation at impacts, improve the controller robustness with respect to approximate satisfaction of condition (61). The system is more sensitive to violation of condition (63), because there is (in frictionless systems) no dissipation to compensate for a bad choice of $\dot{q}_{d}(t)$, since the product $\dot{q}^{T} \xi=0$ during persistent contact (perfect constraint assumption). It would be interesting here to study in which way Coulomb's friction (which adds a dissipative term in the right-hand side of (62)) improves the stability properties.

2. It is clear that the developments in sections 5 and 6 hold each time there is only one joint with clearance and all the other joints are actuated.

3. A similar interpretation as in Figure 7 (b) holds in the trajectory tracking case.

4. The meaning of conditions (61) and (63) is that the desired velocity has to make an acute angle with the normals to the active constraints. The subtlety is that the activeness is taken here in a subset $\mathcal{K}(w)$ of $\mathcal{I}(q)=\left\{i \in\left\{1, . ., m_{u}^{\prime}\right\} \mid f_{i}(q)=0\right\}$ defined as: $\mathcal{K}(w)=\left\{i \in \mathcal{I}(q) \mid w^{T} \nabla f_{i}(q)=0\right\}$. This is depicted in a particular case in Figure 8 (a) where the boundary of $\Phi$ has a singularity (impact at $q$ corresponds to two simultaneous collisions in the joints, called a 2-impact [18, pp.671-672]). So when $q$ is on the boundary of $\Phi, \dot{q}_{d}$ has either to point inside the admissible domain $\Phi$, or be tangent to its boundary. It is noteworthy that when $q \in \operatorname{int}(\Phi)$ then $T_{\Phi}(q)=\mathbb{R}^{n}$ and $T_{T_{\Phi}(q)}(\cdot)=\mathbb{R}^{n}$, hence the choice of $\dot{q}_{d}$ is free, as expected. The fact that $\dot{q}_{d}(\cdot)$ has to be designed such that it tends to keep the trajectories inside $\Phi$, stems from the fact that we basically deal with a motion tracking problem, where the contact interaction force control is not taken into account (which makes another difference with the results in $[21,58]$ in addition to those listed in Remark 10).

\footnotetext{
17 Adapting the desired trajectories has been advocated in [21], then [12,58] in the context of control of fully actuated systems, [34] for particle in billiards, [55] for linear jugglers, [29] for running and walking biped robots.
} 


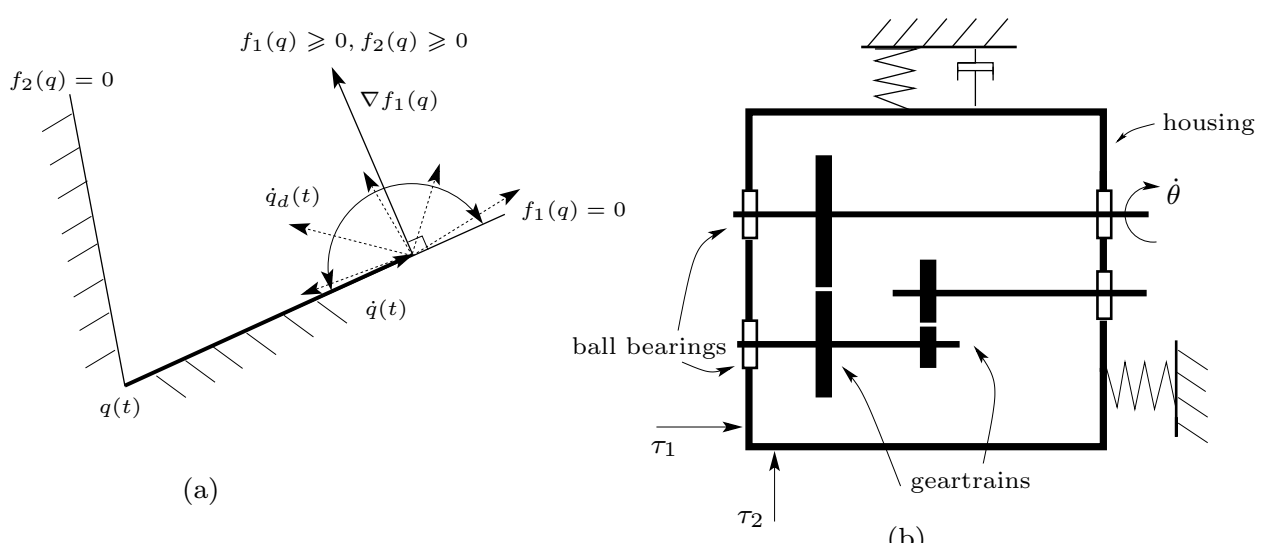

(b)

Fig. 8: (a) The set of desired trajectories. (b) Gearbox system.

5. It is interesting to explore what happens when $V(0)=0 \Leftrightarrow \dot{\tilde{q}}(0)=0$ and $\tilde{q}(0)=0$. According to the above, $V(t)=0$ for all $t \geqslant 0$ if conditions (61) and (63) are satisfied. Since we have assumed that $\dot{q}_{d}(\cdot)$ is continuous, so is $\dot{q}(\cdot)$ and there is no impact. We therefore attain here the limitation of working with a motion controller and continuous desired velocities. However if $V(0) \neq 0$ then impacts may occur despite condition (61) and nothing guarantees from the above analysis, that they vanish.

6. Assume now that $q_{d}(\cdot)$ is absolutely continuous, $\dot{q}_{d}(\cdot)$ is right-continuous of local bounded variations with discontinuities at impact times $t_{k}$. Then

$$
\begin{aligned}
\sigma_{V}\left(t_{k}\right) & =T_{L}\left(t_{k}\right)+T_{L, d}\left(t_{k}\right)+\frac{1}{2}\left[\dot{q}_{d}\left(t_{k}^{+}\right)^{T} \tilde{M}\left(t_{k}\right) \dot{q}_{d}\left(t_{k}^{+}\right)-\frac{1}{2} \dot{q}_{d}\left(t_{k}^{-}\right)^{T} \tilde{M}\left(t_{k}\right) \dot{q}_{d}\left(t_{k}^{-}\right)\right] \\
& -\left[\dot{q}\left(t_{k}^{+}\right) M\left(q\left(t_{k}\right)\right) \dot{q}_{d}\left(t_{k}^{+}\right)-\dot{q}\left(t_{k}^{-}\right) M\left(q\left(t_{k}\right)\right) \dot{q}_{d}\left(t_{k}^{-}\right)\right]
\end{aligned}
$$

where $\tilde{M}\left(t_{k}\right)=M\left(q\left(t_{k}\right)\right)-M\left(q_{d}\left(t_{k}\right)\right), T_{L, d}\left(t_{k}\right)=\frac{1}{2} \dot{q}_{d}\left(t_{k}^{+}\right)^{T} M\left(q_{d}\left(t_{k}\right)\right) \dot{q}_{d}\left(t_{k}^{+}\right)-\frac{1}{2} \dot{q}_{d}\left(t_{k}^{-}\right)^{T} M\left(q_{d}\left(t_{k}\right)\right) \dot{q}_{d}\left(t_{k}^{-}\right)$ is the desired kinetic energy loss, $T_{L}\left(t_{k}\right)$ is the kinetic energy loss. If $\dot{q}_{d}(\cdot)$ obeys the same impact law as $\dot{q}(\cdot)$, then $T_{L, d}\left(t_{k}\right) \leqslant 0$. Assume that $\tilde{q}(0)=0$ and $\dot{\tilde{q}}(0)=0, f(q(0))>0$. Then $V(t)=0 \Rightarrow \tilde{q}(t)=0$ and $\dot{\tilde{q}}(t)=0$ for all $t \in\left[0, t_{0}\right)$. From (66) one has $\sigma_{V}\left(t_{0}\right)=0$ since both $\dot{q}(\cdot)$ and $\dot{q}_{d}(\cdot)$ jump at $t_{0}$ with the same impact mapping. Let condition (63) hold, then $V(\cdot)$ keeps vanishing during persistent contact. We infer that $\tilde{q}(t)=0$ and $\dot{\tilde{q}}(t)=0$ for all $t \geqslant 0$. The next step is to analyse the closed-loop behaviour with initial tracking error. The idea is that if $V(0)>0$ then the third and fourth terms between brackets in (66) may be upperbounded by a function $\epsilon(V(0))$ of the initial error, with $\epsilon(0)=0$. We conjecture that if impacts are separated enough (existence of a uniform large enough dwell-time, whose size depends on $V(0))$, then local stability may be shown, though the semi-negativeness of $\dot{V}(\cdot)$ outside impacts is a weak property. For the sake of briefness this is left as a future work. We nevertheless point out two cases in which impacts are separated: $e_{\mathrm{n}}=0$ (plastic collisions) and $e_{\mathrm{n}}=1$ (elastic collisions). In the first case, an impact is followed by a persistent contact phase. In the second case, it follows from [7, Proposition 4.11] that there exists a dwell-time separating impacts, which depends on the initial velocity.

7. The application of other passivity-based controllers (which are designed for the tracking control of unconstrained Lagrangian systems) is prone to the same difficulties as those mentioned in Remark 10.

8. It is clear that the developments in sections 5 and 6 hold each time there is only one joint with clearance and all the other joints are actuated. The case of multiple clearances is more involved, though the numerical results in [4] indicate that PD, feedback linearization and passivity-based controllers possess remarkable robustness when applied to a four-bar system with one, two or three joint clearances. We may consider the system in Figure 7 (a), with joints $J_{1}, J_{2}$ and $J_{3}$ actuated, and dynamics in (41). 
Mimicking section 5, let us apply the same controller as (57) to subsystem $S_{1}$ in (41) (a):

$$
\tau_{1}=M_{1}\left(q_{1}\right) \ddot{q}_{1, d}+C_{1}\left(q_{1}, \dot{q}_{1}\right) \dot{q}_{1, d}+G_{1}\left(q_{1}\right)-K_{v} \dot{\tilde{q}}_{1}-K_{p} \tilde{q}_{1} .
$$

We are led to analyse the stability with the Lyapunov candidate function (see (59) and (44)), that is $V\left(\tilde{q}_{1}, \dot{\tilde{q}}_{1}, q_{4}, q_{5}, \dot{q}_{4}, \dot{q}_{5}\right)=\frac{1}{2} \dot{\tilde{q}}_{1}^{T} M_{1}\left(q_{1}\right) \dot{\tilde{q}}_{1}+\frac{1}{2} \tilde{q}_{1}^{T} K_{p} \tilde{q}_{1}+\frac{1}{2} \dot{q}_{4}^{T} M_{4} \dot{q}_{4}+U_{4}\left(q_{4}\right)+\frac{1}{2} \dot{q}_{5}^{T} M_{5} \dot{q}_{5}+U_{5}\left(q_{5}\right)$. After few calculations we obtain $\sigma_{V}\left(t_{k}\right)=T_{L}\left(t_{k}\right)-\dot{q}_{1, d}\left(t_{k}\right)^{T} M_{1}\left(q_{1}\right) \sigma_{\dot{q}_{1}}\left(t_{k}\right)$ instead of $(60)$, where $T_{L}\left(t_{k}\right)$ denotes the system's kinetic energy loss. The variation of the term $\dot{q}_{1, d}\left(t_{k}\right)^{T} M_{1}\left(q_{1}\right) \sigma_{\dot{q}_{1}}\left(t_{k}\right)$ which by the impact dynamics equals $\dot{q}_{1, d}\left(t_{k}\right)^{T} \mathcal{M}_{14}\left(q_{1}\left(t_{k}\right), q_{4}\left(t_{k}\right)\right) p_{\mathrm{n}, u, 14}\left(t_{k}\right)$, is not easy to characterize through a simple condition as (61). During persistent contact phases, both controllers in (67) and (43) are equal, and doing the same calculations as in section 5 we obtain along the closed-loop system's trajectories: $\dot{V}(t) \leqslant-\dot{\tilde{q}}_{1}^{T} K_{v} \dot{\tilde{q}}_{1}-\dot{q}_{1, d}(t)^{T} \mathcal{M}_{14}\left(q_{1}, q_{4}\right) \lambda_{\mathrm{n}, 14}(t)$. Again, the second term in the right-hand side is not guaranteed to be non positive without further constraints on the desired trajectory. In case of regulation the desired velocity $\dot{q}_{d}$ vanishes, and we can follow the same lines as in section 5 . Same ideas can be applied to the six-bar system in Figure 6 (a) with dynamics in (26) (27) (28), where one may choose to apply a torque $\tau_{2}$ at joint $J_{4}$, or not, and to the linkage in Figure 3 . In such cases it will be crucial to analyse the system's performance as a function of how many and where actuators and sensors are mounted, and of clearances size. Indeed these parameters will change the set of closed-loop fixed points, hence the precision of the system. Getting back to trajectory tracking: this preliminary analysis suggests that slow enough desired trajectories, or desired trajectories which switch between zero and non zero (with a dwell-time allowing for $V$ to be a non monotonic Lyapunov function) should guarantee some kind of closed-loop stability property, even in the presence of multiple clearances.

\subsubsection{Further investigations}

The same question as in section 5.4 is of interest, however time-varying desired trajectories render the closed-loop stability analysis more complex. An exhaustive numerical analysis on four-bar systems as in Figure 4 (b) when clearances are present at $J_{2}, J_{3}$ and $J_{4}$, while $J_{1}$ is actuated, is made in [4]. It demonstrates that passivity-based and feedback linearization schemes, possess remarkable and surprizing robustness with respect to clearances, in the sense that even high frequency desired trajectories do not destabilize the closed-loop system (but the precision drastically decreases ). As shown in [4, Appendix D] on the one-DoF prismatic joint in Figure 2 (b), a rigorous stability analysis is prone to serious difficulties. Finally another problem is to investigate how the material of section 3 may be extended to trajectory tracking. In other words, given a tracking controller, which conditions on the desired trajectory should be fullfilled so that persistent contact conditions hold? We may start from the subsystem (21) assuming that all the conditions of Proposition 2 hold.

\section{Conclusions}

Systems with joint clearance occupy a particular place within the set of controlled Lagrangian systems with unilateral constraints and impacts. Clearances are hard disturbances which augment the system's dimension and add collisions and contact forces. Hence the major issues for the control of such systems, are the underactuation and the fact that the available output usually consists of partial positions and velocities, because measuring the whole state of the system with joint clearance is often technologically and economically impossible. There are two main control objectives: design "simple" feedback controllers which possess some robustness w.r.t. mechanical play, or design specific controllers which "compensate" for clearances and guarantee high accuracy. Most likely there does not exist a universal controller for systems with joint clearance, because the choice of a control input depends on many parameters like system's dimension, the number of joints with clearances, the size of the clearances, the contact parameters (restitution coefficients, friction), the precision and the number of sensors, the control objective -regulation, tracking, robustness, accuracy improvement-, the degree of actuation, etc. In this paper we have reviewed and analysed three important families of feedback controllers: impactless with persistent 
contact, using impacts in a juggling systems framework, and PD+ for regulation and tracking. Let us end with some open issues and comments (the list being obviously non exhaustive):

1. (State observers) Existing state observers $[56,80]$ measure the whole position vector $q$ and estimate $\dot{q}$, which does not really help in our case. As an example consider the mechanism in Figure 4 (b), actuated at $J_{1}$ and with clearances at the other joints. Is it possible to design an observer which estimates part of the state of subsystem $S_{2}=\left(\mathbf{B}_{2}, \mathbf{B}_{3}\right)$ (sensors may be mounted at some joints), from measurements of the state of subsystem $S_{1}=\left(\mathbf{B}_{1}\right)$ ? Certainly contact phases and impacts have to be used (see $[8,56]$ for observability through the impacts).

2. Which control method (persistent contact control, juggling control) is the less stringent from the actuation degree point of view?

3. (Finite-time convergence) Finite-time convergence controllers are ubiquitous in systems with unilateral constraints: dead-beat controllers for juggling systems [22,52, 53, 57, 90,91], finite-time Lyapunov stable fixed points and sliding-mode control for biped robots $[27,37,64,84]$. In some cases however they may not be adapted to systems with clearances because of the too small size of the clearances (that would imply very high resolution sensors). Controllers which are robust with respect to clearances, or which are not destabilized by impacts and switching dynamics, may be preferred.

4. The framework developed in [73] to find Lyapunov functions solving suitable optimization problems, could be extended to the control problem of section 5 .

5. (Positive, switching, finite-time controls) Control with signed (non negative) inputs stems from the unilaterality of the constraint and is present in sections 3 and 4 . They may be signed impulsive inputs (in case of jugglers), or signed bounded inputs (in case of persistent contact). An interesting path is to investigate in which way the canonical dynamics in (32) could be used in the framework of persistent contact control of section 3. Also the problem of stabilization using controllers which switch between several kinds of sub-controllers (persistent contact, with impacts, PD+), could enlarge the scope of applications of the above results, see Remark 6. Finite-time convergent schemes (like sliding-mode control [18, Section 7.5.5]) would be worth investigating as well. The time-discretization is also an important subject for contact tasks.

6. Some systems like four-bar rocker-rocker mechanisms, have intrinsically bounded generalized coordinates (link angles). Could such a property be used for control when joint clearances are present ? The mere question: under which conditions is a clearance-free controller robust with respect to the presence of joint clearances ? remains largely open (a purely numerical analysis is performed in [4]).

7. The main differences with other classes of unilaterally constrained mechanical systems (biped robots, manipulators with environment, pull/push robotic systems) are on one hand that the gap functions usually take very small values in joints with clearance. Hence the contact detection requires very high precision and resolution sensors. On the other hand the control context is not the same since clearances are viewed as disturbances inducing decrease in the system's performance (while the constraints are part of the system's dynamics in biped robots, for instance). The consequence is that the degree of actuation and the measured output vary a lot from one class of systems to another.

8. (Adapted desired trajectories) Adapting the desired trajectories seems unavoidable in many instances of control of unilaterally constrained systems with impacts. It has been advocated in $[21]$, then $[12,58]$ in the context of control of fully actuated systems, [34] for particle in billiards, [55] for linear jugglers, [29] for running and walking biped robots.

9. (Accuracy) The set $\mathcal{E}$ of equilibrium points that stems from the $\mathrm{PD}+$ gravity compensation input (section 5), and consequently the accuracy of the closed-loop system, are influenced by the degree of actuation. Consider as an example the 6-bar system in Figure 6 (a): how does the addition/deletion of an actuator at joint $J_{4}$ (the joints $J_{1}, J_{2}$ and $J_{6}$ being actuated) modify the set of closed-loop equilibria ? Studying the influence of the clearance-free system degree of actuation on the closed-loop accuracy (in addition to stability), is a crucial topic.

10. (Vibrations active control) When the clearances are small enough, it is intuitively clear that the dynamical effects due to clearances could be assimilated to vibrations. Vibration analysis associated with ball bearings clearance may be found in $[28,41]$. This could be used in a gearbox system as in Figure 8 (b) with clearances in the ball bearings and in the geartrains and imposed angular velocity 
$\dot{\theta}$, with control inputs $\tau_{1}$ and $\tau_{2}$ designed to compensate for the vibration effects on the housing dynamics. However this departs from the point of view adopted in this article and consequently this is not analysed.

11. (Compliant contacts) As long as one assumes that actuators cannot apply a control action during the collisions, the usefulness of compliant rheological models for control design, is not clear. Thus except if contact times allow for some control action during the impacts, considering deformations at contact does not bring much to the control design problem.

12. (3D joints) The overall dynamical framework adopted in this paper encompasses three-dimensional joints, provided the modeling step for the choice of the gap functions has been solved. Indeed three-dimensional revolute, prismatic or spherical joints involve plane/plane, or cylinder/bore (journal/bearing), or sphere/ contacts, which are particularly uneasy to model due to conformal surfaces and other issues (see for instance [70,71] for comparisons of various rheological models of cylinder/bore contact, whose conclusion is that all the tested models yield quite different outcomes).

13. (Flexible systems) Flexibilities in bodies and in joints need to be analyzed in a control context, either to test the robustness of feedback controllers for rigid-body rigid-joint systems, or to incorporate them in the control design.

14. (Robustness) Nothing has been said about robustness of the various controllers with respect to disturbances and uncertainties other than clearances. The controllers presented in sections $3,4,5$ and 6 possess quite different features, consequently they should be robust with respect to different kinds of perturbations.

15. (Friction) We have focussed on dry, frictionless contacts. Friction may play a negative role in certain cases (especially in prismatic joints where singularities like Painlevé paradoxes may occur [18]). When only revolute joints are considered, frictional effects may be less important [4].

16. (Discrete-time controllers) How is the stability influenced by the implementation of discrete-time versions of the presented controllers ? For instance, how to guarantee that the discretized version of persistent contact controllers, still guarantee persistent contact?

\section{A About the canonical form (32)}

To start let us rewrite the dynamics in (25) (a) (b) (c) as:

$$
\left\{\begin{array}{l}
\dot{x}_{1}=x_{2} \\
\dot{x}_{2}=-M_{2}^{-1} G_{2}\left(x_{1}\right)+M_{2}^{-1} \mathcal{M}_{21}\left(x_{1}, x_{3}\right) \lambda_{\mathrm{n}, u, 12}+M_{2}^{-1} \mathcal{M}_{23}\left(x_{1}, x_{3}\right) \lambda_{\mathrm{n}, u, 23} \\
\dot{x}_{3}=x_{4} \\
\left.\dot{x}_{4}=\left(\begin{array}{cc}
M_{1}\left(x_{3}\right)^{-1} & 0 \\
0 & M_{3}\left(x_{3}\right)^{-1}
\end{array}\right)\left\{\left(\begin{array}{l}
-F_{1}\left(x_{3}, x_{4}\right) \\
-F_{3}\left(x_{3}, x_{4}\right)
\end{array}\right)+\mathcal{D}\left(x_{1}, x_{3}\right)\right)^{\dagger} \hat{\tau}+\left(I_{4}-\mathcal{D}\left(x_{1}, x_{3}\right)^{\dagger} \mathcal{D}\left(x_{1}, x_{3}\right)\right) \tilde{\tau}+\mathcal{M}\left(x_{1}, x_{3}\right) \lambda_{\mathrm{n}, u}\right\},
\end{array}\right.
$$

where we used the decomposition $\left.\tau=\mathcal{D}\left(x_{1}, x_{3}\right)\right)^{\dagger} \hat{\tau}+\left(I_{4}-\mathcal{D}\left(x_{1}, x_{3}\right)^{\dagger} \mathcal{D}\left(x_{1}, x_{3}\right)\right) \tilde{\tau}, x_{1}=q_{2}, x_{2}=\dot{q}_{2}, x_{3}=\left(q_{1}^{T}, q_{3}^{T}\right)^{T}$, $x_{4}=\left(\dot{q}_{1}^{T}, \dot{q}_{3}^{T}\right)^{T}$ (in $(31)$ we set $\left.\hat{\tau}=\bar{\tau}-\mathcal{F}(q, \dot{q})\right)$. This system is of the form $\dot{x}=f(x)+g(x) u+h(x) \lambda_{\mathrm{n}}, u=\left(\hat{\tau}^{T}, \tilde{\tau}^{T}\right)^{T}$, and we can write $g(x) u=\hat{g}(x) \hat{\tau}+\tilde{g}(x) \tilde{\tau}$. If some conditions about I/O linearization are satisfied, there exists a (local) diffeomorphism such that $z=Z(x)$ transforms $\dot{x}=f(x)+\hat{g}(x) \hat{\tau}$, into $\dot{z}=F(z)+\hat{G}(z) \hat{\tau}$, with $F(z)=\frac{\partial Z}{\partial x} f(x)=\left(\begin{array}{c}-M_{2}^{-1} G_{2}\left(z_{1}\right) \\ z_{4} \\ \mathcal{F}(z) \\ \Xi_{z}\left(\xi, z_{3}\right)\end{array}\right)$, where $\mathcal{F}(z)$ stems from $\mathcal{F}(q, \dot{q})$ in $(30), \hat{G}(z)=\frac{\partial Z}{\partial x} \hat{g}(x)=\left(\begin{array}{c}0 \\ 0 \\ \left.\mathcal{D}\left(x_{3}, x_{4}\right) \mathcal{D}\left(x_{1}, x_{3}\right)\right)^{\dagger} \\ 0\end{array}\right)$, where $\hat{\tau}$ does not appear in the $\xi$ dynamics. Here we have $z=\left(z_{1}^{T}, z_{2}^{T}, z_{3}^{T}, z_{4}^{T}, z_{5}^{T}\right)^{T}=\left(x_{1}^{T}, x_{2}^{T}, z_{3}^{T}, z_{4}^{T}, \xi^{T}\right)^{T}=\left(q_{2}^{T}, \dot{q}_{2}^{T}, z_{3}^{T}, z_{4}^{T}, \xi^{T}\right)^{T}$, see $(32)$. The same diffeomorphism yields $\tilde{G}(z)=\frac{\partial Z}{\partial x} \tilde{g}(x)=\left(\begin{array}{c}0 \\ 0 \\ 0 \\ \Xi_{\tau}(z)\end{array}\right)$, this time $\tilde{\tau}$ does not appear in the $z_{4}$-dynamics (recall that $z_{4}=\dot{y}$ ),

because $\mathcal{D}(q)\left(I_{4}-\mathcal{D}(q)^{\dagger} \mathcal{D}(q)\right)=0$, see (30). Now why should $\Xi_{\tau}(z) \neq 0$ ? The state transformation being diffeomorphic, it is desirable that the controllability properties of both systems (in $x$ and in $z$ coordinates) remain unchanged. If $\Xi_{\tau}(z)=0$ obviously this is not the case (having $\Xi_{\tau}(z)=0$ would correspond to underactuated systems $S_{1}$ and $S_{3}$ in $(25$ ) (b) and 
(c)). Finally the matrix $\Xi_{\lambda}(z)$ in (32) is non zero in general, because impacts may induce jumps in all velocities, including zero dynamics.

\section{References}

1. Acary, V.: Projected event-capturing time-stepping schemes for nonsmooth mechanical systems with unilateral contact and Coulomb's friction. Computer Methods in Applied Mechanics and Engineering 256, 224-250 (2013)

2. Acary, V., Brogliato, B.: Numerical Methods for Nonsmooth Dynamical Systems. Applications in Mechanics and Electronics, Lecture Notes in Applied and Computational Mechanics, vol. 35. Springer-Verlag, Berlin Heidelberg (2008)

3. Addi, K., Brogliato, B., Goeleven, D.: A qualitative mathematical analysis of a class of linear variational inequalities via semi-complementarity problems: applications in electronics. Mathematical Programming 126(1), 31-67 (2011)

4. Akhadkar, N., Acary, V., Brogliato, B.: Analysis of collocated feedback controllers for four-bar planar mechanisms with joint clearances. Multibody System Dynamics 38(2), 101-136 (2016)

5. Ames, A.D., Galloway, K., Sreenath, K., Grizzle, J.W.: Rapidily exponentially stabilizing control Lyapunov functions and hybrid zero dynamics. IEEE Transactions on Automatic Control 59(4), 876-891 (2014)

6. Aoustin, Y., Formalskii, A.M.: Modeling, control and simulation of upward jump of a biped. Multibody System Dynamics 29, 425-445 (2013)

7. Ballard, P.: Formulation and well-posedness of the dynamics of rigid body systems with perfect unilateral constraints. Phil. Trans. R. Soc. London A 359(1789), 2327-2346 (2001)

8. Baumann, M., Leine, R.I.: A synchronization-based state observer for impact oscillators using only collision time information. Int. Journal of Robust and Nonlinear Control 26, 2542-2563 (2016)

9. di Bernardo, M., Budd, C.J., Champneys, A.R., Kowalczyk, P.: Piecewise-Smooth Dynamical Systems, Applied Mathematical Sciences, vol. 163. Springer Verlag, London (2008)

10. Bernstein, D.S.: Matrix Mathematics. Theory, Facts, and Formulas with Application to Linear Systems Theory. Princeton University Press (2005)

11. Blumentals, A., Brogliato, B., Bertails-Descoubes, F.: The contact problem in Lagrangian systems subject to bilateral and unilateral constraints, with or without sliding Coulomb's friction: a tutorial. Multibody System Dynamics 38, 43-76 (2016)

12. Bourgeot, J.M., Brogliato, B.: Tracking control of Lagrangian complementarity systems. International Journal of Bifurcation and Chaos 15(6), 1839-1866 (2005)

13. Brammer, R.: Controllability in linear autonomous systems with positive controllers. SIAM. J. Control 10(2), 339-353 (1972)

14. Brogliato, B.: Some perspectives on the analysis and control of complementarity systems. IEEE Transactions on Automatic Control 48(6), 918-935 (2003)

15. Brogliato, B.: Absolute stability and the Lagrange-Dirichlet theorem with monotone multivalued mappings. Systems and Control Letters 51(5), 343-353 (2004)

16. Brogliato, B.: Inertial couplings between unilateral and bilateral holonomic constraints in frictionless Lagrangian systems. Multibody System Dynamics 29(3), 289-325 (2013)

17. Brogliato, B.: Kinetic quasi-velocities in unilaterally constrained Lagrangian mechanics with impacts and friction. Multibody System Dynamics 32(2), 175-216 (2014)

18. Brogliato, B.: Nonsmooth Mechanics. Models, Dynamics and Control, third edn. Communications and Control Engineering. Springer International Publishing Switzerland (2016)

19. Brogliato, B., Lozano, R., Maschke, B., Egeland, O.: Dissipative Systems Analysis and Control. Theory and Applications, 2nd edn. Communcations and Control Engineering. Springer Verlag, London (2007)

20. Brogliato, B., Mabrouk, M., Zavala-Rio, A.: On the controllability of linear juggling mechanical systems. Systems and Control Letters 55, 350-367 (2006)

21. Brogliato, B., Niculescu, S.I., Orhant, P.: On the control of finite-dimensional mechanical systems with unilateral constraints. IEEE Transactions on Automatic Control 42(2), 200-215 (1997)

22. Brogliato, B., Zavala-Rio, A.: On the control of complementary-slackness mechanical juggling systems. IEEE Transactions on Automatic Control 45(2), 235-246 (2000)

23. Byrnes, C.I., Isidori, A.: Asymptotic stabilization of minimum phase nonlinear systems. IEEE Transactions on Automatic Control 36(10), 1122-1337 (1991)

24. Camlibel, M.: Popov-Belevitch-Hautus type tests for the controllability of linear complementarity systems. Systems and Control Letters 56(5), 381-387 (2007)

25. Champneys, A.R., Varkonyi, P.R.: The Painlevé paradox in contact mechanics. IMA Journal of Applied Mechanics 81, $538-588(2016)$

26. Chang, S.L., Tsai, L.W.: On the redundant-drive backlash-free robotic mechanisms. ASME Journal of Mechanical Design 115, 247-254 (1993)

27. Chang, T., Hurmuzlu, Y.: Sliding control without reaching phase and its application to bipedal locomotion. ASME Journal of Dynamic Systems, Measurement, and Control 115(3), 447-455 (1993)

28. Changqing, B., Qingyu, X.: Dynamic model of ball bearings with internal clearance and waviness. Journal of Sound and Vibration 294, 23-48 (2006)

29. Chevallereau, C., Westervelt, E.R., Grizzle, J.W.: Asymptotically stable running for a five-link, four-actuator, planar bipedal robot. International Journal of Robotics Research 24(6), 431-464 (2005) 
30. Duarte, F., Machado, J.T.: Describing function of two masses with backlash. Nonlinear Dynamics 56, 409-413 (2009)

31. Facchinei, F., Pang, J.S.: Finite-Dimensional Inequalities and Complementarity Problems, Volume I. Springer Series in Operations Research. Springer-Verlag, Berlin Heidelberg (2003)

32. Flores, P., Ambrosio, J., Claro, J.P., Lankarani, H.: Kinematics and Dynamics of Multibody Systems with Imperfect Joints, Lecture Notes in Applied and Computational Mechanics, vol. 34. Springer Berlin Heidelberg (2008)

33. Flores, P., Koshy, C., Lankarani, H., Ambrosio, J., Claro, J.: Numerical and experimental investigation on multibody systems with revolute joint clearance joints. Nonlinear Dynamics 65(4), 383-398 (2011)

34. Galeani, S., Menini, L., Potini, A., Tornambè, A.: Trajectory tracking for a particle in elliptical billiards. International Journal of Control 81(2), 189-213 (2008)

35. Glocker, C.: Set-Valued Force Laws, Lecture Notes in Applied Mechanics, vol. 1. Springer Verlag, Berlin Heidelberg (2001)

36. Glocker, C.: An introduction to impacts. In: J. Haslinger, G. Stavroulakis (eds.) Nonsmooth Mechanics of Solids, no. 485 in CISM Courses and Lectures, pp. 45-102. International Centre for Mechanical Sciences, Springer Verlag, Wien-New York (2006)

37. Grizzle, J., Abba, G., Plestan, F.: Asymptotically stable walking for biped robots: analysis via systems with impulse effects. IEEE Transactions on Automatic Control 46(1), 51-64 (2001)

38. Guan, Y., Li, M., Lim, T., Jr, W.S.: Comparative analysis of actuator concepts for active gear pair vibration control. Journal of Sound and Vibration 269, 273-294 (2004)

39. Hiriat-Urruty, J.B., Lemaréchal, C.: Fundamentals of Convex Analysis. Grundlehren text Editions. Springer-Verlag, Berlin Heidelberg (2000)

40. Hurmuzlu, Y., Génot, F., Brogliato, B.: Modeling, stability and control of biped robots-a general framework. Automatica 40(10), 1647-1664 (2004)

41. Jang, G., Jeong, S.: Vibration analysis of a rotating system due to the effect of ball bearing waviness. Journal of Sound and Vibration 269, 709-726 (2004)

42. Koshy, C., Flores, P., Lankarani, H.: Study of the effect of contact force model on the dynamic response of mechanical systems with dry clearance joints: computational and experimental approaches. Nonlinear Dynamics 73(1), 325-338 (2013)

43. Krinner, A., Thümmel, T.: Non-smooth behaviour of a linkage mechanism with revolute clearance joints. In: New Advances in Mechanisms, Transmissions and Applications. Proceedings of the Second Conference MeTrApp 2013, Mechanisms and Machine Science, vol. 17, pp. 233-241 (2014)

44. Lagerberg, A., Egardt, B.: Backlash estimation with application to automotive powertrains. IEEE Transactions on Control Systems Technology 15(3), 483-493 (2007)

45. Lampaert, V., Swevers, J., Al-Bender, F.: Modification of the Leuven friction model structure. IEEE Transactions on Automatic Control 47(4), 683-687 (2002)

46. Lancaster, P., Tismenetsky, M.: The Theory of Matrices, 2nd edn. Academic Press, Orlando, Florida (1985)

47. Leine, R.I., van de Wouw, N.: Stability and Convergence of Mechanical Systems with Unilateral Constraints, Lecture Notes in Applied and Computational Mechanics, vol. 36. Springer Verlag, Berlin Heidelberg (2008)

48. Lewis, A.: Simple mechanical control systems with constraints. IEEE Transactions on Automatic Control 45(8), 1420$1436(2000)$

49. Li, P., Chen, W., Li, D., Yu, R.: A novel transition model for lubricated revolute joints in planar multibody systems. Multibody System Dynamics 36, 279-294 (2016)

50. Liljebäck, P., Pettersen, K.Y., Stavdhal, O., Gravdhal, J.T.: Hybrid modelling and control of obstacle-aided snake robot locomotion. IEEE Transactions on Robotics 26(5), 781-799 (2010)

51. Liljebäck, P., Pettersen, K.Y., Stavdhal, O., Gravdhal, J.T.: Snake Robots. Modelling, Mechatronics, and Control. Advances in Industrial Control. Springer-Verlag, London (2013)

52. Lynch, K., Black, C.: Recurrence, controllability, and stabilization of juggling. IEEE Transactions on Robotics and Automation 17(2), 113-124 (2001)

53. Mata-Jimenez, M., Brogliato, B.: Analysis of PD and nonlinear control of mechanical systems with dynamic backlash. Journal of Vibration and Control 9(1), 119-156 (2003)

54. McClamroch, N., Wang, D.: Feedback stabilization and tracking of constrained robots. IEEE Transactions on Automatic Control 33(5), 419-426 (1988)

55. Menini, L., Possieri, C., Tornambé, A.: On the computation of the continuous-time reference trajectory for mechanical juggling systems. In: Proc. IEEE 54th Annual Conference on Decision and Control, pp. 145-150. Osaka, Japan (2015)

56. Menini, L., Tornambé, A.: Velocity observers for non-linear mechanical systems subject to non-smooth impacts. Automatica 38, 2169-2175 (2002)

57. Menini, L., Tornambè, A.: Control of (otherwise) uncontrollable linear mechanical systems through non-smooth impacts. Systems and Control Letters 49(4), $311-322$ (2003)

58. Morarescu, C.I., Brogliato, B.: Trajectory tracking control of multiconstraint complementarity Lagrangian systems. IEEE Transactions on Automatic Control 55(6), 1300-1313 (2010)

59. Moreau, J.J.: Unilateral contact and dry friction in finite freedom dynamics. In: J.J. Moreau, P. Panagiotopoulos (eds.) Nonsmooth Mechanics and Applications, no. 302 in CISM Courses and Lectures, pp. 1-82. International Centre for Mechanical Sciences, Springer Verlag, Wien-New York (1988)

60. Müller, A.: Internal preload control of redundantly actuated parallel manipulators-its application to backlash avoiding control. IEEE Transactions on Robotics 21(4), 668-677 (2005)

61. Nguyen, N., Brogliato, B.: Multiple Impacts in Dissipative Granular Chains, Lecture Notes in Applied and Computational Mechanics, vol. 72. Springer Berlin Heidelberg (2014) 
62. Nordin, M., Galic, J., Gutman, P.: New models for backlash and gear play. Int. Journal of Adaptive Control and Signal Processing 11, 49-63 (1997)

63. Nordin, M., Gutman, P.: Controlling mechanical systems with backlash-a survey. Automatica 38, 1633-1649 (2002)

64. Oza, H.B., Orlov, Y.V., Spurgeon, S.K.: Finite time stabilization of a perturbed double integrator with unilateral constraints. Mathematics and Computers in Simulation 95(1), 200-212 (2014)

65. Özdemir, M.: Removal of singularities in the inverse dynamics of parallel robots. Mechanism and Machine Theory 107, 71-86 (2017)

66. Paden, B., Panja, R.: Globally asymptotically stable 'PD+' controller for robot manipulators. International Journal of Control 47(6), 1697-1712 (1988)

67. Paoli, L.: Vibro-impact problems with dry friction-Part I: Existence result. SIAM J. on Mathematical Analysis 47(5), 3285-3313 (2015)

68. Paoli, L.: Vibro-impact problems with dry friction-Part II: Tangential contacts and frictional catastrophes. SIAM J. on Mathematical Analysis 48(2), 1272-1296 (2016)

69. Paoli, L., Schatzman, M.: Penalty approximation for dynamical systems submitted to multiple non-smooth constraints. Multibody System Dynamics 8(3), 347-366 (2002)

70. Pereira, C., Ramalho, A., Ambrosio, J.: A critical overview of internal and external cylinder contact force models. Nonlinear Dynamics 63(4), 681-697 (2011)

71. Pereira, C.M., Ramalho, A.L., Ambrosio, J.A.: Applicability domain of internal cylindrical contact force models. Mechanism and Machine Theory 78, 141-157 (2014)

72. Pfeiffer, F., Glocker, C.: Multibody Dynamics with Unilateral Contacts. Nonlinear Science. Wiley (1996)

73. Posa, M., Tobenkin, M., Tedrake, R.: Stability analysis and control of rigid-body systems with impacts and friction. IEEE Transactions on Automatic Control 61(6), 1423-1437 (2016)

74. Reyhanoglu, M., van der Schaft, A., McClamroch, N., Kolmanovsky, I.: Dynamics and control of a class of underactuated mechanical systems. IEEE Transactions on Automatic Control 44(9), 1663-1671 (1999)

75. Rodic, A., Vukobratovic, M., Addi, K., Dalleau, G.: Contribution to the modeling of nonsmooth multipoint contact dynamics of biped locomotion-theory and experiments. Robotica 26, 157-175 (2007)

76. Saperstone, S.: Global controllability of linear systems with positive controls. SIAM J. Control 11(3), 417-423 (1973)

77. Saperstone, S., Yorke, J.: Controllability of linear oscillatory systems using positive controls. SIAM. J. Control 9(2), 253-262 (1971)

78. Serra, D., Satici, A.C., Ruggiero, F., Lippiello, V., Siciliano, B.: An optimal trajectory planner for a robotic batting task: The table tennis example. In: Proc. 13th International Conference on Informatics in Control, Automation and Robotics., pp. 90-101. Lisbon, Portugal (2016)

79. Spong, M.W., Kelly, R., Ortega, R.: Comments on "Adaptive manipulator control: A case study". IEEE Transactions on Automatic Control 35(6), 761-762 (1990)

80. Tanwani, A., Brogliato, B., Prieur, C.: Observer design for unilaterally constrained Lagrangian systems: A passivitybased approach. IEEE Transactions on Automatic Control 61(9), 2386-2401 (2016)

81. Thuemmel, T., Rossner, M.: Introduction to modelling and parameter identification methodology of linkages by measurements and simulation. In: Proceedings of 13rd World Congress in Mechanisms and Machine Science, Guanajuato, Mexico, 19-25 June, pp. IMD-123 (2011)

82. Thümmel, T., Ginzinger, L.: Measurements and simulations of a crank and rocker mechanism including friction, clearance and impacts. In: Proceedings of IXth Intern. Conf. on the Theory of Machines and Mechanisms, Liberec, Czech Republic, August 31-September 02. Liberec, Czech Republic (2004)

83. Varedi, S., Daniali, H., Dardel, M., Fathi, A.: Optimal dynamic design of a planar slider-crank mechanism with a joint clearance. Mechanism and Machine Theory 86, $191-200$ (2015)

84. Westervelt, E., Grizzle, J., Koditschek, D.: Hybrid zero dynamics of planar biped walkers. IEEE Transactions on Automatic Control 48(1), 42-56 (2003)

85. Wieber, P.B., Chevallereau, C.: Online adaptation of reference trajectories for the control of walking systems. Robotics and Autonomous Systems 54(7), 559-566 (2006)

86. Wojtyra, M.: Modeling of static friction in closed-loop kinematic chains-uniqueness and parametric sensitivity problems. Multibody System Dynamics (2016). Doi:10.1007/s11044-016-9535-6

87. van de Wouw, N., Leine, R.I.: Attractivity of equilibrium sets of systems with dry friction. Nonlinear Dynamics 35(1), 19-39 (2008)

88. Xiao, H., Shao, Y., Brennan, M.: On the contact stiffness and nonlinear vibration of an elastic body with a rough surface in contact with a rigid surface. European Journal of Mechanics A/Solids 49, 315-328 (2015)

89. Yan, S., Xiang, W., Zhang, L.: A comprehensive model for 3D revolute joints with clearances in mechanical systems. Nonlinear Dynamics 80(1), 309-328 (2015)

90. Zavala-Rio, A., Brogliato, B.: On the control of a one degree-of-freedom juggling robot. Dynamics and Control 9, 67-90 (1999)

91. Zavala-Rio, A., Brogliato, B.: Direct adaptive control design for one-degree-of-freedom complementary-slackness jugglers. Automatica 37, 1117-1123 (2001) 